\title{
Article
}

\section{Separation of Stellar Populations by an Evolving Bar: Implications for the Bulge of the Milky Way}

Debattista, Victor P., Ness, Melissa, González, Oscar A., Freeman, K., Zoccali, Manuela and Minniti, Dante

Available at http://clok.uclan.ac.uk/18047/

Debattista, Victor P., Ness, Melissa, González, Oscar A., Freeman, K., Zoccali, Manuela and Minniti, Dante (2017) Separation of Stellar Populations by an Evolving Bar: Implications for the Bulge of the Milky Way. Monthly Notices of the Royal Astronomical Society, 469 (2). pp. 1587-1611. ISSN 0035-8711

It is advisable to refer to the publisher's version if you intend to cite from the work. http://dx.doi.org/10.1093/mnras/stx947

For more information about UCLan's research in this area go to http://www.uclan.ac.uk/researchgroups/ and search for <name of research Group>.

For information about Research generally at UCLan please go to http://www.uclan.ac.uk/research/

All outputs in CLoK are protected by Intellectual Property Rights law, including Copyright law. Copyright, IPR and Moral Rights for the works on this site are retained by the individual authors and/or other copyright owners. Terms and conditions for use of this material are defined in the policies page.

\section{CLoK}

Central Lancashire online Knowledge www.clok.uclan.ac.uk

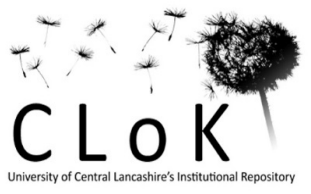




\title{
Separation of stellar populations by an evolving bar: implications for the bulge of the Milky Way
}

\author{
Victor P. Debattista, ${ }^{1 \star}$ Melissa Ness, ${ }^{2}$ Oscar A. Gonzalez, ${ }^{3}$ K. Freeman, ${ }^{4}$ \\ Manuela Zoccali ${ }^{5,6}$ and Dante Minniti ${ }^{6,7,8}$ \\ ${ }^{1}$ Jeremiah Horrocks Institute, University of Central Lancashire, Preston PR1 2HE, UK \\ ${ }^{2}$ Max-Planck-Institut für Astronomie, Königstuhl 17, D-69117 Heidelberg, Germany \\ ${ }^{3}$ UK Astronomy Technology Centre, Royal Observatory, Blackford Hill, Edinburgh EH9 3HJ, UK \\ ${ }^{4}$ Research School of Astronomy and Astrophysics, Mount Stromlo Observatory, Cotter Road, Weston Creek ACT 2611, Australia \\ ${ }^{5}$ Instituto de Astrofísica, Facultad de Física, Pontificia Universidad Católica de Chile, Av. Vicuña Mackenna 4860, 782-0436 Macul, Santiago, Chile \\ ${ }^{6}$ The Millennium Institute of Astrophysics (MAS), Av. Vicuña Mackenna 4860, 782-0436 Macul, Santiago, Chile \\ ${ }^{7}$ Departamento de Ciencias Fisicas, Universidad Andres Bello, Republica 220, Santiago, Chile \\ ${ }^{8}$ Vatican Observatory, I-00120 Vatican City State, Italy
}

Accepted 2017 April 19. Received 2017 April 18; in original form 2016 April 15

\begin{abstract}
We present a novel interpretation of the previously puzzling different behaviours of stellar populations of the Milky Way's bulge. We first show, by means of pure $N$-body simulations, that initially co-spatial stellar populations with different in-plane random motions separate when a bar forms. The radially cooler populations form a strong bar, and are vertically thin and peanut-shaped, while the hotter populations form a weaker bar and become a vertically thicker box. We demonstrate that it is the radial, not the vertical, velocity dispersion that dominates this evolution. Assuming that early stellar discs heat rapidly as they form, then both the in-plane and vertical random motions correlate with stellar age and chemistry, leading to different density distributions for metal-rich and metal-poor stars. We then use a high-resolution simulation, in which all stars form out of gas, to demonstrate that this is what happens. When we apply these results to the Milky Way we show that a very broad range of observed trends for ages, densities, kinematics and chemistries, that have been presented as evidence for contradictory paths to the formation of the bulge, are in fact consistent with a bulge which formed from a continuum of disc stellar populations which were kinematically separated by the bar. For the first time, we are able to account for the bulge's main trends via a model in which the bulge formed largely in situ. Since the model is generic, we also predict the general appearance of stellar population maps of external edge-on galaxies.
\end{abstract}

Key words: Galaxy: bulge-Galaxy: evolution-Galaxy: formation-Galaxy: structuregalaxies: bulges - galaxies: kinematics and dynamics.

\section{INTRODUCTION}

The origin of the bulge of the Milky Way has been the subject of considerable discussion. On the one hand, many lines of evidence now point to the fact that the Milky Way hosts a bar (de Vaucouleurs 1964; Peters 1975; Cohen \& Few 1976; Liszt \& Burton 1980; Gerhard \& Vietri 1986; Mulder \& Liem 1986; Binney et al. 1991; Nakada et al. 1991; Whitelock \& Catchpole 1992; Weiland et al. 1994; Paczynski et al. 1994; Dwek et al. 1995; Zhao, Rich \& Spergel 1996; Sevenster 1996; Binney, Gerhard \& Spergel 1997; Nikolaev \& Weinberg 1997; Stanek et al. 1997;

^E-mail: vpdebattista@gmail.com
Hammersley et al. 2000). This leads to the opportunity for secular evolution to have sculpted the centre of the Milky Way into the bulge we see today. Evidence of this comes from the morphology and kinematics of the bulge. The X-shape of the red clump distribution in the bulge is manifested by the bimodal distribution of their distances (McWilliam \& Zoccali 2010; Nataf et al. 2010; Saito et al. 2011; Wegg \& Gerhard 2013; Gonzalez et al. 2015). This distribution is produced by looking through the near- and far-side corners of a peanut-shaped bulge (e.g. Li \& Shen 2012). Such box-/peanut-shaped (B-/P-shaped) bulges are produced by the buckling instability of bars (Raha et al. 1991; Merritt \& Sellwood 1994; Bureau \& Athanassoula 2005; Debattista et al. 2006) or by orbit trapping (Combes \& Sanders 1981; Combes et al. 1990; Quillen 2002; Quillen et al. 2014) and is observed in many external 
galaxies. Li \& Shen (2012) showed that a buckled bar model is fully able to account for this morphology in the Milky Way. Meanwhile data from the BRAVA, ARGOS and APOGEE surveys have shown that the bulge is cylindrically rotating (Howard et al. 2008; Ness et al. 2013b, 2016b), which is the typical velocity field produced by bars. A more detailed comparison of a barred simulation with kinematics from the BRAVA survey (Howard et al. 2008; Kunder et al. 2012) by Shen et al. (2010) noted that the velocity and velocity dispersion profiles can be reproduced provided a slowly rotating bulge component constitutes less than 8 percent of the disc mass. Thus, these properties all favour the presence of a bulge formed by the vertical thickening of the bar [see the reviews of Kormendy \& Kennicutt (2004) and Fisher \& Drory (2016)].

The alternative path to the formation of bulges is their accretion as part of the hierarchical growth of galaxies (Kauffmann, White \& Guiderdoni 1993; Guedes et al. 2013). Various lines of evidence favouring the existence of such a component in the Galactic bulge have been put forth. Photometric studies of resolved stellar populations have generally found uniformly old stars across the bulge, typically older than $10 \mathrm{Gyr}$ (Ortolani et al. 1995; Kuijken \& Rich 2002; Zoccali et al. 2003; Ferreras, Wyse \& Silk 2003; Sahu et al. 2006; Clarkson et al. 2008, 2011; Brown et al. 2010; Valenti et al. 2013; Calamida et al. 2014). For instance, Clarkson et al. (2011) estimate that any component younger than 5 Gyr must account for less than 3.4 per cent of the bulge. The Milky Way bulge also exhibits a metallicity gradient in the vertical direction (Zoccali et al. 2008; Gonzalez et al. 2011; Johnson et al. 2011, 2013). This vertical gradient was thought to be impossible if the bulge formed via the buckling instability. Evidence for an accreted bulge was also inferred from the old and metal-poor RR Lyrae at the centre of the Galaxy, which Dékány et al. (2013) showed traced a more axisymmetric, weakly barred shape than the red clump stars. Lastly, the velocity dispersion of metal-poor stars on the minor-axis above $|b|$ $\simeq 4^{\circ}$ becomes flat, while that of metal-rich stars continues declining (Babusiaux 2016), suggesting the existence of a second component.

One interpretation of these seemingly conflicting results is that the Milky Way has a compound bulge (Athanassoula 2005; Debattista et al. 2005), with both a secular B/P bulge and an accreted classical bulge present (Babusiaux et al. 2010; Hill et al. 2011; Zoccali et al. 2014). Many external galaxies have been found to host compound bulges. For instance, Méndez-Abreu et al. (2014) found that $\sim 30$ per cent of their sample of face-on barred galaxies showed signs of multiple bulge components, while Erwin et al. (2015) presented a sample of nine galaxies with composite bulges (see also Erwin et al. 2003). Thus, composite bulges are not rare, and it is conceivable that the Milky Way has one too. A compound bulge can easily produce the vertical metallicity gradient observed via the variation of the relative importance of two components (Grieco et al. 2012).

One of the challenges that pure secular evolution models have faced is why the properties of the bulge should be different for different populations (usually defined via the metallicity of the stars). Since gravity cannot act differently on stars of different metallicities, a number of studies have postulated that the different stellar populations represent different structural components, or perhaps different regions of the initial disc. Martinez-Valpuesta \& Gerhard (2013) demonstrate that, contrary to naive expectations, buckling can generate a vertical metallicity gradient, consistent with that observed, out of steep radial gradients. Di Matteo et al. (2014) instead consider pure $N$-body simulations including a classical bulge and find that stars initially in the disc as far out as the outer Lindblad resonance of the bar contribute to the boxy bulge, comprising up to
30 per cent of stars at high latitudes. In these scenarios, assuming that the initial disc has a declining metallicity profile, a puzzling observation is that the X-shape is weak or absent in the metal-poor stars (Ness et al. 2012; Uttenthaler et al. 2012; Rojas-Arriagada et al. 2014). Bekki \& Tsujimoto (2011) instead considered the evolution of a thin+thick disc model, where the thick disc was metalpoor and the thin disc was metal-rich. In this way, they were able to build a vertical metallicity gradient in the bulge. Di Matteo et al. (2015) argued that a single thin disc model is unable to produce the observed split red clump in the metal-rich stars and no split in the metal-poor red clump stars.

As argued by Di Matteo et al. (2015), we do not expect that a single thin stellar disc is able to explain the full range of data uncovered for stars in the bulge because there is nothing to distinguish different chemical populations. Like those authors, we find that multiple discs are needed to explain the observational data. However, we show that it is not primarily the disc thickness that drives the differences between stellar populations in the bulge, but rather the initial random motion in the disc plane, a situation which arises naturally in a disc during its early rapid growth phase. In this paper, we consider both purely collisionless $N$-body simulations comprised of multiple stellar populations with different kinematics and a simulation with star formation, in order to understand how the structure of the Milky Way's bulge arises. We discover that stellar populations separate (further) in the presence of a bar, a process we refer to as kinematic fractionation. We explore the implications of this separation for the morphology, kinematics, ages, chemistry and microlensing of the bulge.

This paper is organized as follows. In Section 2, we present a simple dynamical interpretation for why kinematic fractionation occurs. Section 3 presents a study based on pure $N$-body simulations of kinematic fractionation, showing that the radial velocity dispersion is the main driving factor in the separation and that the vertical velocity dispersion plays a smaller role. We then demonstrate the same behaviour in a simulation with gas and star formation in Section 4. Section 5 compares the trends in the resultant bulge of this simulation with those in the Milky Way's bulge, showing that all the observed trends can be reproduced, with only an additional 5 per cent hot component (a stellar halo) needed to match the kinematics at low metallicity. We also predict new trends for testing for kinematic fractionation in the Milky Way. Section 6 discusses these results including making predictions for the metallicity and age distributions of $\mathrm{B} / \mathrm{P}$ bulges in edge-on galaxies, and ends with a summary of our conclusions.

\section{THEORETICAL CONSIDERATIONS}

Below we present models in which stellar populations with different initial in-plane kinematics, but identical density distribution, separate as the bar forms and grows. We refer to this behaviour as kinematic fractionation, in analogy with chemical fractionation produced by phase transitions. The key result of these simulations is that the populations with larger initial in-plane random motion go on to become thicker and less peanut-shaped, and to host weaker bars.

Before presenting these simulations, we seek to understand qualitatively why radially hotter discs become vertically thicker. Merritt \& Sellwood (1994) showed that bending instabilities in discs occur when the vertical frequency of stars, $v$, exceeds the frequency with which they encounter a vertical perturbation, allowing them to respond in phase with a perturbation, thereby enhancing it. Bars are the most important source of bending instabilities for two reasons. First, 
because of their intrinsic geometry, bars enforce $m=2$ (saddleshaped) bends, which generally are very vigorous. Secondly, bars raise the in-plane velocity dispersion, but do not substantially alter the vertical dispersion unless they suffer bending instabilities. Why does this matter? Consider the case of a vertical $m=2$ perturbation rotating at frequency $\Omega_{p}$, the pattern speed of the bar. The frequency at which stars encounter this perturbation is $2\left(\Omega-\Omega_{p}\right)$, where $\Omega$ is the mean angular frequency of the stars. Thus, stars will support the perturbation as long as

$v>2\left(\Omega-\Omega_{p}\right)$,

enabling it to grow inside co-rotation. If we separate disc stars into two populations based on their radial random motion, we can label population ' $h$ ' (hot) as the one with the larger radial velocity dispersion and population ' $c$ ' (cool) the one with the smaller dispersion. It is well known that the asymmetric drift, $\Delta V \equiv V_{\text {circ }}-\left\langle V_{\phi}\right\rangle \simeq x \sigma_{R}^{2} / V_{\text {circ }}$ [e.g. Binney \& Tremaine (1987), section 4.2.1(a)], where $V_{\text {circ }}$ is the circular velocity and $\left\langle V_{\phi}\right\rangle$ is the mean streaming velocity of the stars and $x$ is a factor that depends on the density distribution and orientation of the velocity ellipsoid; $-1<x<1$ at one disc scalelength. Thus

$\Omega \simeq \Omega_{\text {circ }}-\frac{x}{R V_{\text {circ }}} \sigma_{R}^{2}$,

where $\Omega_{\text {circ }}$ is the frequency of circular orbits at radius $R$. Therefore, $\Omega_{h}<\Omega_{c}$ : raising $\sigma_{R}$ lowers $\Omega$. While we have assumed near axisymmetry to make this argument, the underlying principle, that hotter populations rotate more slowly, holds also when the perturbation is stronger since the mean streaming motion is just one component supporting a population against collapse. Stars in population ' $h$ ' satisfy inequality 1 at a smaller $v$ than those in population 'c'. A smaller $v$ is achieved when the maximum height, $z_{\max }$, that the star reaches is larger. Since, as a population thickens, $v$ declines faster than $\Omega$ (Appendix A presents orbit integrations which demonstrate this result), a bend in population ' $h$ ' will saturate at a larger $z_{\max }$ than in population ' $c$ '. We conclude that in general radially hotter populations can thicken more than radially cooler ones.

This separation of different kinematic populations does not end at the buckling instability. Bars transfer angular momentum to the halo slowing down in the process (Weinberg 1985; Debattista \& Sellwood 1998, 2000; Athanassoula 2002; O'Neill \& Dubinski 2003). With $\Omega_{p}$ declining and $\sigma_{R}$ rising, the right-hand side of equation (1) drops, allowing more stars to respond in phase with further perturbations. As long as the bar is slowing, therefore, the disc will continue to thicken at different rates for different radial dispersion populations, allowing the separation of populations to persist in subsequent evolution. As examples of this behaviour, MartinezValpuesta, Shlosman \& Heller (2006) found that slowing bars that had buckled already can buckle again, while Debattista et al. (2006) was able to induce buckling in an otherwise stable bar by slowing it down with an impulsively imposed torque.

\section{DEMONSTRATION USING PURE $N$-BODY MODELS}

The initial conditions usually employed in pure $N$-body simulations studying disc galaxy evolution have generally set up a disc composed of a single distribution function, equivalent to a single stellar population. In reality, discs are composed of multiple populations of different ages with different dispersions; even in the Solar neighbourhood, the radial, tangential and vertical velocity dispersions are all a function of age (e.g. Wielen 1977; Nordström et al. 2004;
Holmberg, Nordström \& Andersen 2009; Aumer \& Binney 2009; Casagrande et al. 2011). In the early Universe, disc galaxies were subject to significant internal turmoil (e.g. Glazebrook 2013), and had lower mass, which resulted in rapid heating. As a consequence, in young galaxies, the stellar random motion increases rapidly with age over a relatively short period of time. Alternatively, early discs may form directly hot, with subsequent populations forming in cooler, thinner distributions (Kassin et al. 2012; Bird et al. 2013; Stinson et al. 2013; Wisnioski et al. 2015; Grand et al. 2016). The outcome of this upside-down evolution is similar: discs with random motions increasing as a function of age.

In order to explore the effect of the age dependence of random motions on the evolution of different populations, we first study the behaviour of multipopulation discs by means of carefully controlled pure $\mathrm{N}$-body (i.e. gasless) simulations where the initial conditions are comprised of multiple superposed stellar discs with different initial kinematics. We run two types of simulations: one in which multiple stellar discs are exactly co-spatial but have different in-plane kinematics (described in Section 3.1), and another set with only two discs, with different initial heights (described in Section 3.2).

\subsection{Co-spatial discs}

In the first set of simulations, we build systems in which the initial disc is comprised of five separate populations with different inplane velocity dispersions but identical density distribution. We set up initial conditions using GalaCtics (Kuijken \& Dubinski 1995; Widrow \& Dubinski 2005; Widrow, Pym \& Dubinski 2008). We construct a model consisting of an exponential disc and a NavarroFrenk-White (NFW) halo (Navarro, Frenk \& White 1996), with no bulge component. We truncate this NFW halo as follows:

$\rho(r)=\frac{2^{2-\gamma} \sigma_{h}^{2}}{4 \pi a_{h}^{2}} \frac{1}{\left(r / a_{h}\right)^{\gamma}\left(1+r / a_{h}\right)^{3-\gamma}} C\left(r ; r_{h}, \delta r_{h}\right)$,

(Widrow et al. 2008). Here, $C\left(r ; r_{h}, \delta r_{h}\right)$ is a cutoff function to smoothly truncate the model at a finite radius, and is given by:

$C\left(r ; r_{h}, \delta r_{h}\right)=\frac{1}{2} \operatorname{erfc}\left(\frac{r-r_{h}}{\sqrt{2} \delta r_{h}}\right)$.

Our choice of parameters is $\sigma_{h}=400 \mathrm{~km} \mathrm{~s}^{-1}, a_{h}=16.7 \mathrm{kpc}$, $\gamma=0.873, r_{h}=100 \mathrm{kpc}$ and $\delta r_{h}=25 \mathrm{kpc}$.

We build model discs with an exponential profile:

$\Sigma(R, z)=\Sigma_{0} \exp \left(-R / R_{\mathrm{d}}\right) \operatorname{sech}^{2}\left(z / z_{\mathrm{d}}\right)$,

where $R_{\mathrm{d}}$ is the disc scalelength and $z_{\mathrm{d}}$ is the scaleheight. Our discs have $M_{\mathrm{d}}=2 \pi \Sigma_{0} R_{\mathrm{d}}^{2}=5.2 \times 10^{10} \mathrm{M}_{\odot}, R_{\mathrm{d}}=2.4 \mathrm{kpc}$ and $z_{\mathrm{d}}=250 \mathrm{pc}$. The rotation curve resulting from this set of parameters is shown in the top panel of Fig. 1. We construct five versions of this disc density, with different radial velocity dispersion profiles:

$\sigma_{R}^{2}(R)=\sigma_{R 0}^{2} \exp \left(-R / R_{\sigma}\right)$.

We vary the central velocity dispersion, $\sigma_{R 0}$, fixing $R_{\sigma}$ to $2.5 \mathrm{kpc}$. We select five values of $\sigma_{R 0}, 190 \mathrm{~km} \mathrm{~s}^{-1}$ (disc D1), $165 \mathrm{~km} \mathrm{~s}^{-1}$ (disc D2), $140 \mathrm{~km} \mathrm{~s}^{-1}$ (disc D3), $115 \mathrm{~km} \mathrm{~s}^{-1}$ (disc D4) and $90 \mathrm{~km} \mathrm{~s}^{-1}$ (disc D5). This large range of $\sigma_{R 0}$ was chosen to enhance the effect of kinematic fractionation; the evolution of further models with a narrower range of $\sigma_{R 0}$ is qualitatively similar to what we will show here. The middle panel of Fig. 1 shows the different radial velocity dispersion profiles of the five discs. Although we vary the in-plane kinematics of the initial discs, the vertical density profile of each disc is identical and therefore the vertical velocity dispersion, $\sigma_{z}$, shown in the bottom panel of Fig. 1, is too. We use $6 \times 10^{6}$ particles 

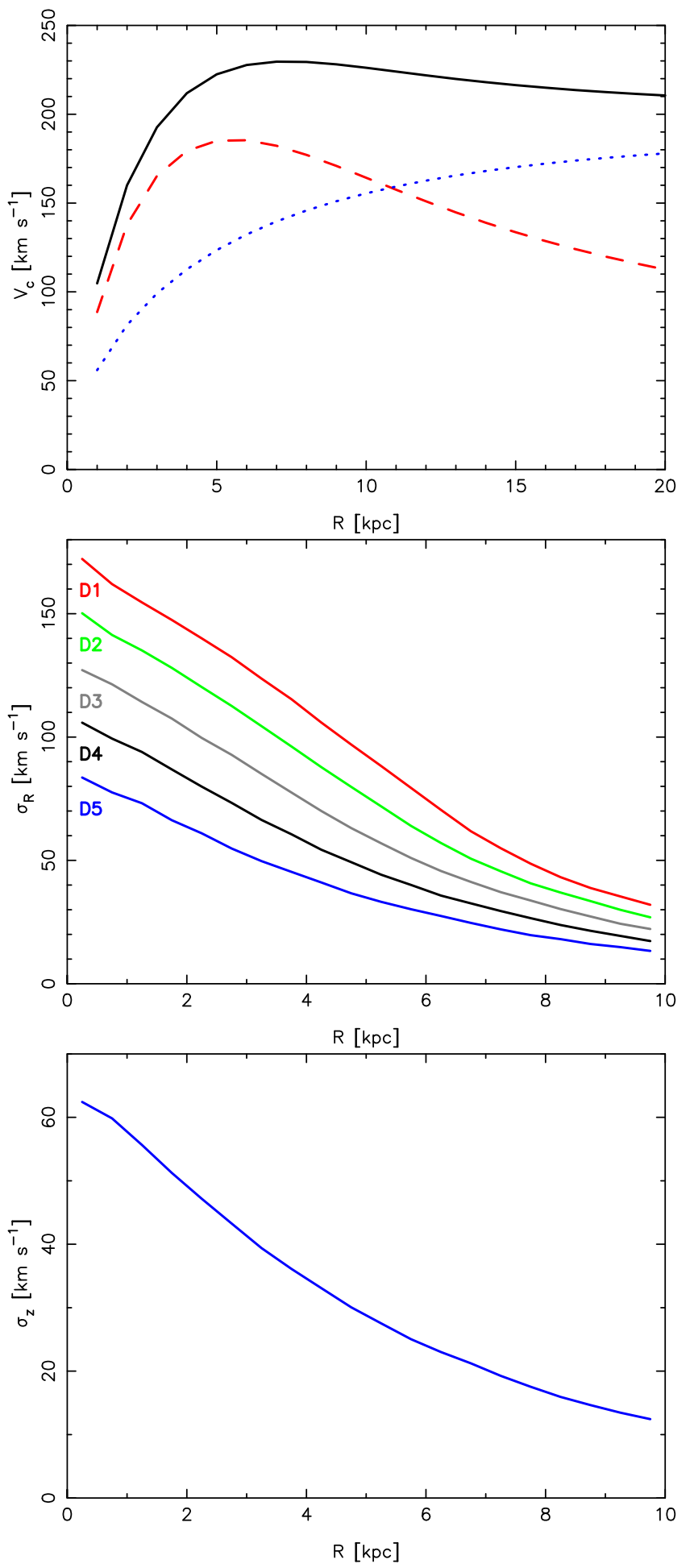

Figure 1. Top: the rotation curve of the pure $N$-body models D1-D5. The dotted (blue) curve shows the halo contribution, the dashed (red) curve shows that of the stars and the solid (black) curve shows the full rotation curve. Middle: radial velocity dispersions, $\sigma_{R}$, for discs D1 (red) to D5 (blue). Bottom: vertical velocity dispersion, $\sigma_{z}$, of the disc in model D5. Models D1-D4 have $\sigma_{z}$ almost identical to this.

in each disc and $4 \times 10^{6}$ particles in the halo, giving particles of mass $\simeq 1.1 \times 10^{4} \mathrm{M}_{\odot}($ disc $)$ and $\simeq 1.7 \times 10^{5} \mathrm{M}_{\odot}$ (halo).

Once we build the five discs, we construct a further two compound discs which are superpositions of different subsamples of
D1-D5. In model CU ('compounded uniformly'), we sample each of the discs equally. Thus, we extract from each one-fifth of their particles and join them together to form a new composite disc with ratio D1:D2:D3:D4:D5 set to 1:1:1:1:1. The second disc, model CL ('compounded linearly'), samples the discs assuming a linearly decreasing contribution from D1 to D5 in the ratio 5:4:3:2:1. These composite systems mimic a system with a continuum of stellar populations, in the case of $\mathrm{CU}$ with a constant star formation rate, while CL corresponds to a declining star formation rate (assuming that the kinematically hotter populations are older). We therefore refer to each of disc D1-D5 in models CU and CL as different populations.

Because the overall mass distribution is unchanged by the resampling, by the linearity of the Boltzmann and Poisson equations, the composite discs CL and CU are also in equilibrium. We evolve these models using PKDGRAV (Stadel 2001), with a particle softening of $\epsilon=50$ and $100 \mathrm{pc}$ for star and halo particles, respectively. Our base time-step is $\Delta t=5 \mathrm{Myr}$ and we refine time-steps such that each particle's time-step satisfies $\delta t=\Delta t / 2^{n}<\eta \sqrt{\epsilon / a_{g}}$, where $a_{g}$ is the acceleration at the particle's current position; we set $\eta=0.2$. Our gravity calculation uses an opening angle of the tree code $\theta=0.7$.

\subsubsection{Evolution of single-population discs}

Before considering the multipopulation discs, we demonstrate that the single-population discs evolve very differently from each other using the two most different models, discs D1 and D5. Fig. 2 shows their evolution. The top panel of Fig. 2 shows the bar amplitude, defined as

$A_{\mathrm{bar}}=\left|\frac{\sum_{i} m_{i} e^{2 i \phi_{i}}}{\sum_{i} m_{i}}\right|$

where the sums extend over all star particles in a given disc population, $m_{i}$ is the mass of the $i$ th particle (which is the same for all star particles in these pure $N$-body simulations) and $\phi_{i}$ is its cylindrical angle. In both models, the bar forms by $1 \mathrm{Gyr}$, although the bar in model D1 is best characterized as a very weak oval at this point. Later, the two bars reach a semimajor axis of $\sim 6.5 \mathrm{kpc}$. The bar in model D5 buckles at $\sim 3.7 \mathrm{Gyr}$, at which point its amplitude decreases. In model D1 instead an axisymmetric buckling occurs at $0.4 \mathrm{Gyr}$, before the bar forms. We measure the radial profile of the root-mean-square height of star particles, $h_{z}$, and average this profile to a radius of $2 \mathrm{kpc},\left\langle h_{z}\right\rangle$; the second panel from the top shows its time evolution. Whereas $\left\langle h_{z}\right\rangle$ evolves mildly in model D5 until the bar buckles, in model D1 $\left\langle h_{z}\right\rangle$ rises very sharply from 250 to $\sim 700 \mathrm{pc}$, while the bar is still forming; thereafter it barely evolves any further. In model D5 instead the rise in $\left\langle h_{z}\right\rangle$ is initially driven by slow heating by the bar and abruptly by the buckling. The third and fourth panels show the evolution of the radial and vertical velocity dispersions averaged over the same radial range. In model D5, $\left\langle\sigma_{R}\right\rangle$ rises rapidly once the bar forms, while $\left\langle\sigma_{z}\right\rangle$ increases only slightly. The buckling in model D5 increases $\left\langle\sigma_{z}\right\rangle$ at the expense of $\left\langle\sigma_{R}\right\rangle$. Instead, in model D1 a large fraction of the random motion in the plane is transformed into vertical random motion. At later times, both $\left\langle\sigma_{R}\right\rangle$ and $\left\langle\sigma_{z}\right\rangle$ evolve very little.

The vertical evolution of a system composed of a single population is therefore strongly determined by the radial velocity dispersion before bar formation. A radially hot disc efficiently transforms a large fraction of its in-plane random motion into vertical random motion. If a hot disc and a cold disc are coincident, a reasonable expectation is that the cold disc drives the formation of a strong bar, which transforms a large part of the hot disc's radial random motion into vertical random motion, thickening the system overall. 


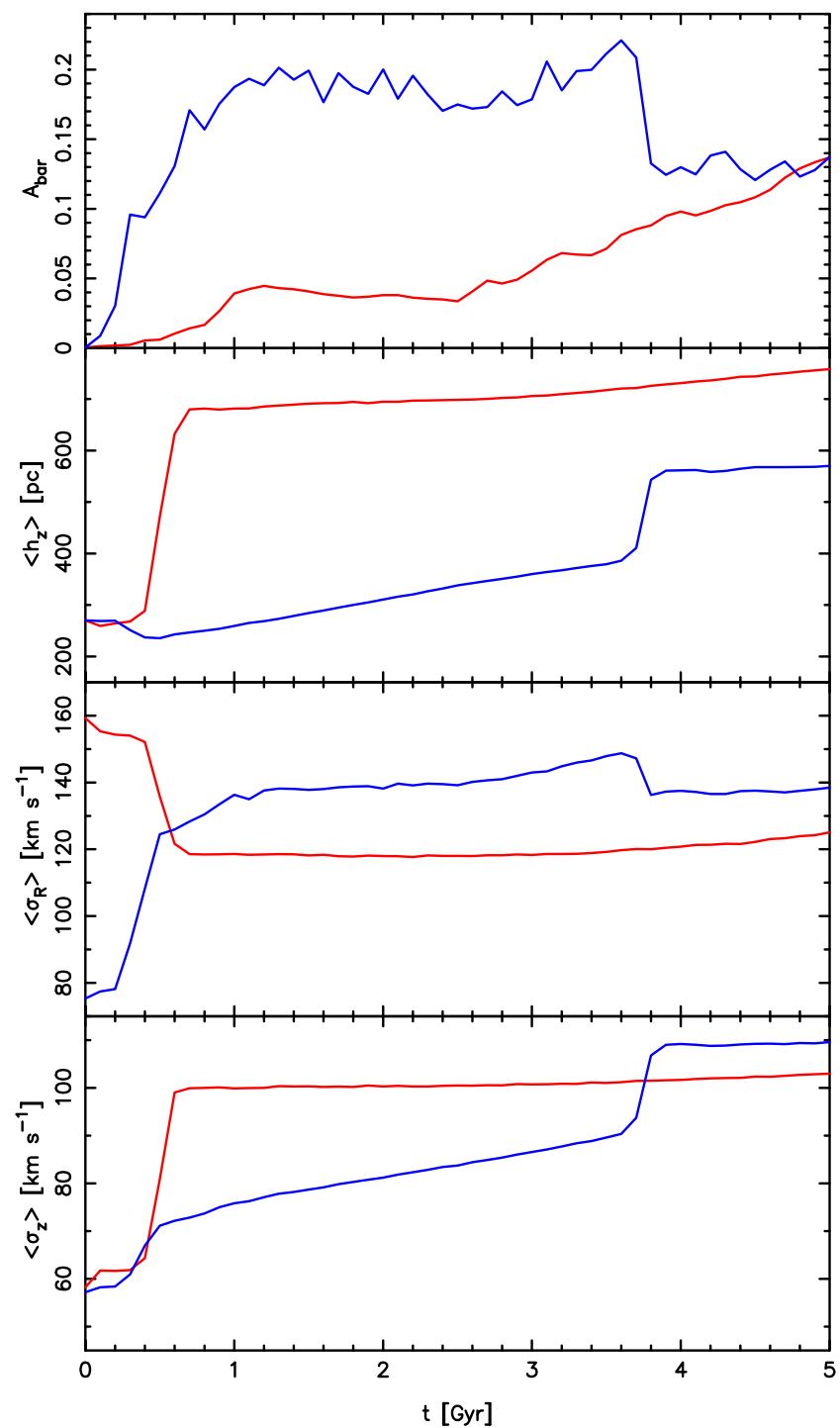

Figure 2. Evolution of the pure $N$-body single-population models with discs D1 (red lines) and D5 (blue lines) when evolved as single-population systems. From top to bottom, we plot the bar amplitude, $A_{\mathrm{bar}}$, the average height, $\left\langle h_{z}\right\rangle$, the average radial velocity dispersions $\left\langle\sigma_{R}\right\rangle$ and the average vertical velocity dispersion $\left\langle\sigma_{z}\right\rangle$. Profiles are averaged in the radial range $R \leq 2 \mathrm{kpc}$ to match the region where $h_{z}$ peaks. The two discs evolve very differently, with disc D1 (the hotter disc) heating vertically at the expense of in-plane random motions.

\subsubsection{Evolution of multipopulation co-spatial discs}

Fig. 3 presents the evolution of model CL (compounded linearly), separately for each disc population. The top panel shows the bar amplitude; the bar is strongest in the coolest population (D5) and weakest in the hottest (D1). This is the case at bar formation ( $t \simeq 1.5 \mathrm{Gyr}$ ) and continues to be so after the strong buckling starting at around $t=2.1 \mathrm{Gyr}$. The average height, $\left\langle h_{z}\right\rangle$, shown in the second panel of Fig. 3, increases the least in population D5, while D1 starts thickening once the bar starts forming, when the disc starts developing bends in the vertical density distribution. Even after buckling saturates, population D1 continues to be the thickest population, as anticipated by the discussion in Section 2. The third row of Fig. 3 shows the average radial velocity dispersion, $\left\langle\sigma_{R}\right\rangle$; this rises rapidly for population D5 at bar formation and then drops

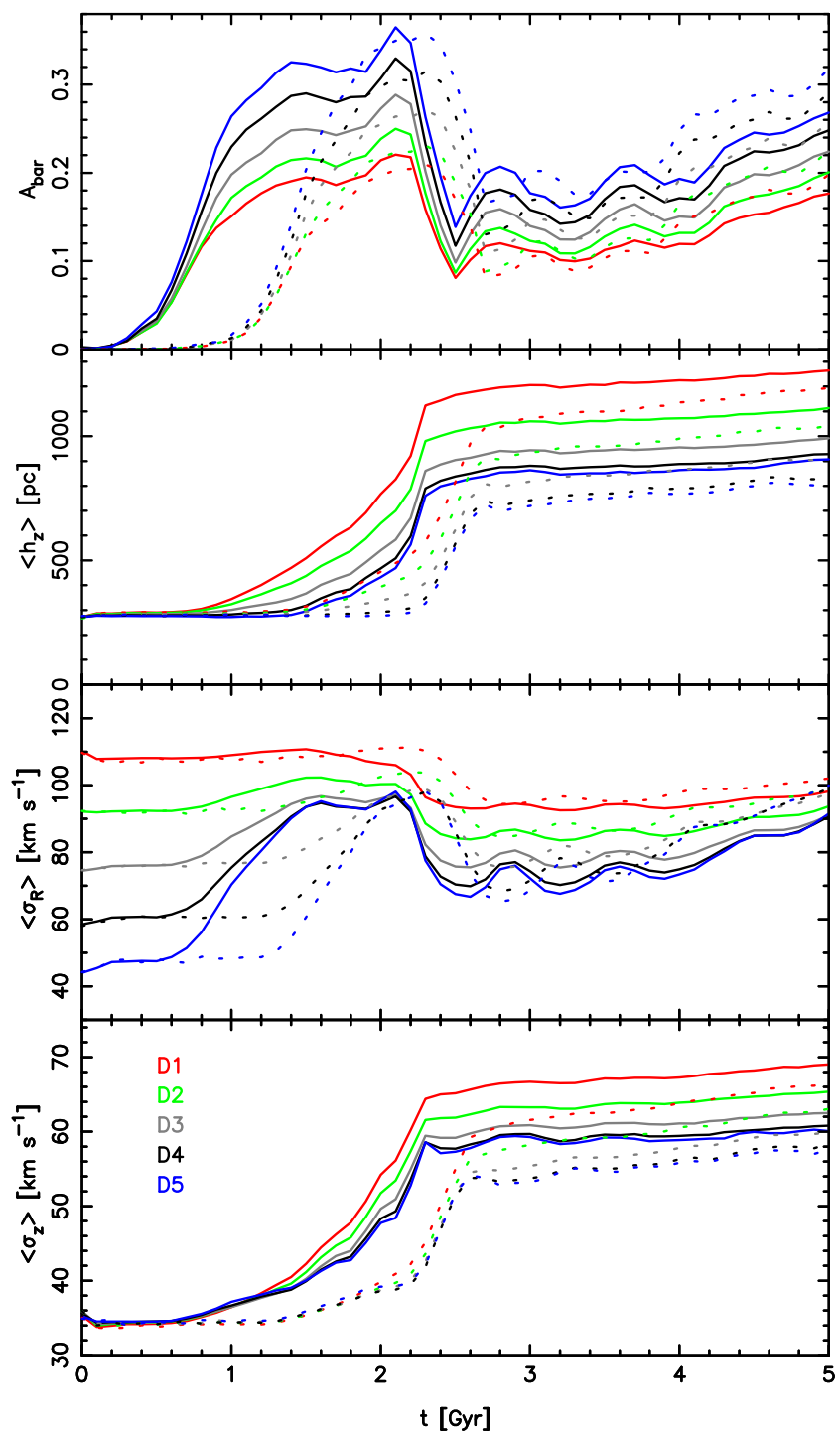

Figure 3. Evolution of the different populations in model CL. Top: bar amplitudes. Upper middle: average heights, $\left\langle h_{z}\right\rangle$. Lower middle: average radial velocity dispersions, $\left\langle\sigma_{R}\right\rangle$. Bottom: average vertical velocity dispersions, $\left\langle\sigma_{z}\right\rangle$. Populations D1-D5 are indicated by red to blue solid lines (respectively). The dotted lines show the evolution of the same system when we employ a quiet start, as described in the text. Profiles are averaged in the radial range $2 \leq R / \mathrm{kpc} \leq 6$, to match the region of peak $h_{z}$. Note the separation of the different populations, both in the plane (in bar strength) and vertically.

at buckling as the bar is weakened, whereas for population D1 bar formation does not change $\left\langle\sigma_{R}\right\rangle$ while buckling radially cools it slightly. The bottom row of Fig. 3 shows the vertical velocity dispersion, $\left\langle\sigma_{z}\right\rangle$, which rises for all populations. While the evolution tends to drive $\left\langle\sigma_{R}\right\rangle$ towards convergence, that of $\left\langle\sigma_{z}\right\rangle$ tends to be parallel for the different populations, even though they are evolving in the same potential. The bar amplitude, height and random motions all vary nearly monotonically with increasing initial in-plane random motions.

We have verified that the evolution, and especially the rapid vertical thickening of the different populations, is indeed driven by the growing bar (rather than being a numerical artefact or imperfect initial conditions) by employing a quiet start (Sellwood 1983), in which 2.5 per cent of the particles from each initial disc population, 
and from the halo, are drawn from the full system. Each chosen particle, with phase space coordinates $\left(x, y, z, v_{x}, v_{y}, v_{z}\right)$, is then replaced by 40 particles as follows:

$$
\left(\begin{array}{c}
x \\
y \\
z \\
v_{x} \\
v_{y} \\
v_{z}
\end{array}\right) \rightarrow \sum_{n=0}^{19} \mathcal{R}\left(\frac{n \pi}{10}\right)\left(\begin{array}{c}
x \\
y \\
z \\
v_{x} \\
v_{y} \\
v_{z}
\end{array}\right)+\sum_{n=0}^{19} \mathcal{R}\left(\frac{n \pi}{10}\right)\left(\begin{array}{c}
x \\
y \\
-z \\
v_{x} \\
v_{y} \\
-v_{z}
\end{array}\right)
$$

where $\mathcal{R}(\phi)$ is the rotation matrix for a rotation by an angle $\phi$ about the $z$-axis. This setup leads to a very low seed $m=2$ perturbation out of which the bar must grow, delaying its formation. The dotted lines in Fig. 3 show that the bar forms about 0.5 Gyr later with this quiet start. The thickening and heating of the different populations is then also delayed, which would not occur if thickening arises for artificial numerical reasons, and must therefore be driven by the bar.

The evolution of model CU (compounded uniformly) is qualitatively similar to that of CL. Because the number of particles in each population is the same in model CU, it makes visual comparison between different populations easier. Fig. 4 presents the final density distribution of each population in model CU. In the face-on view, population D1 has a significantly rounder bar with a weaker quadrupole moment, while the bar in population D5 has a stronger quadrupole moment. None the less, the bar has the same size in all five populations. The difference in the vertical structure is even more striking; while population D1 has a thick centre and a somewhat boxy shape, population D5 is thinner at the centre and thus exhibits a peanut shape. The edge-on unsharp masks in the right column reveal a photometric X-shape in populations D3-D5 which is smeared out in populations D1 and D2.

We have verified that the separation of populations is not dependent on the presence of very hot populations by running a further model comprised of an equal mix of discs D4 and D5, the two coolest discs. The two populations separate to a degree comparable to what they did in model CL. Therefore, kinematic fractionation does not require very hot populations to be present.

\subsection{Evolution of discs with different heights}

In this section, we present four different simulations each containing two discs of different heights. We use the same version of the thicker disc in all four simulations. The thinner discs have the same density distribution and therefore also the same vertical random motions in all four cases, but differ in their radial random motions. In this way, we are able to study the relative importance of vertical and radial random motions to the final $\mathrm{B} / \mathrm{P}$ bulge morphology of the two different populations.

In order to set up initial conditions with discs of different thickness, we again use GALACTICS, which allows two discs with different density distributions to be set up. We choose identically the same halo parameters as in models CL and CU, and again include no bulges in the models. Instead of a pseudo-continuum of populations as in models CL and CU, we use only two discs, one thinner and the other thicker. Both discs have a scalelength $R_{\mathrm{d}}=2.4 \mathrm{kpc}$. Each disc has a mass $M_{\mathrm{d}}=2.6 \times 10^{10} \mathrm{M}_{\odot}$ (i.e. half that of the total disc in models $\mathrm{CL}$ and $\mathrm{CU}$ ) and is represented by $3 \times 10^{6}$ particles. One disc has a scaleheight $z_{\mathrm{d}}=100 \mathrm{pc}$ (thinner disc), while the other has $z_{\mathrm{d}}=400 \mathrm{pc}$ (thicker disc). These two discs are therefore not to be thought of as the thin and thick discs of a galaxy such as the Milky Way. Indeed both discs are considerably thinner than the Milky Way's thick disc and closer to the thin disc (Jurić et al. 2008), which accounts for our terminology 'thinner' and 'thicker' rather than 'thin' and 'thick'. As before, $\sigma_{R}^{2}$ declines exponentially, from $\sigma_{R 0}=90 \mathrm{~km} \mathrm{~s}^{-1}$ for the thicker disc. We produce four thinner disc models. In T1, the thinner disc is radially cooler than the thicker disc, while in models T3 and T4, the thinner disc is radially hotter. In model T2, we set up the thinner disc such that it has almost the same $\sigma_{R}$ profile as the thicker disc. Fig. 5 shows the initial conditions of these systems.

As with models $\mathrm{CU}$ and $\mathrm{CL}$, we evolve these models using PKDGRAV with the same numerical parameters. Fig. 6 compares the final $h_{z}$ profiles of these models. All four thinner disc models start out with the same density distribution. The radial extent and degree to which both discs heat vertically increases from model T1 to T4, i.e. with increasing in-plane random motion of the thinner disc. In models $\mathrm{T} 1$ and T2, the thinner disc remains thinner than the thicker disc, but in models $\mathrm{T} 3$ and $\mathrm{T} 4$, the thinner disc heats to the extent that their height becomes larger than that of the thicker disc at the end of the simulation. Fig. 7 shows the overall evolution of the systems. In models T2 and T3, the bar has equal amplitude in both discs, while the bar is stronger in the thinner disc in $\mathrm{T} 1$ and in the thicker (but radially cooler) disc in model T4. The velocity dispersions increase in all models, with $\left\langle\sigma_{z}\right\rangle$ increasing rapidly in the thinner disc around the time of bar formation. $\left\langle\sigma_{z}\right\rangle$ increases the most in model $\mathrm{T} 4$, for which the thinner disc become vertically hotter than the thicker disc. Buckling occurs in these models at times ranging from 0.3 Gyr (model T1) to 1.1 Gyr (model T4).

Fig. 8 shows face-on and edge-on density maps as well as edgeon unsharp mask images of the thinner and thicker populations in model T4. The thicker population, which, initially, has lower radial velocity dispersion than the thinner population, ends up vertically cooler with a more readily apparent peanut shape, which stands out clearly in the unsharp mask. The thinner disc instead ends up more vertically extended and hosting a weaker bar with a weaker peanut shape than the thicker disc. Thus, it is not the initial height of the disc that is the strongest driver of the final bulge morphology (i.e. whether it is boxy or peanut-shaped), but the in-plane random motions.

\subsection{Summary of the pure $N$-body simulations}

The key insights these pure $N$-body experiments provide can be summarized as follows:

(i) The formation and evolution of a bar causes co-spatial stellar populations, with different initial radial velocity dispersions, to separate. The initially radially hotter populations are lifted to larger heights than the cooler populations, form a weaker bar, and give rise to a boxy/spheroidal shape, not a peanut shape. The cooler populations instead form a peanut shape.

(ii) A thin, radially hot population thickens more than a thick, radially cool population. While it is natural that a radially hotter population will also be vertically hotter and thicker, the initial thickness of the disc is not the main driver of the final morphology. Instead, the initial in-plane random motions are more important in determining the subsequent bulge morphology than is the initial vertical dispersion.

We stress that the initial conditions we have used in these pure $\mathrm{N}$-body simulations are not always meant to represent realistic systems; indeed it would be hard to conceive of a formation scenario which leads to a vertically thicker disc being radially cooler than a thinner disc. The goal of these experiments has been to show the 


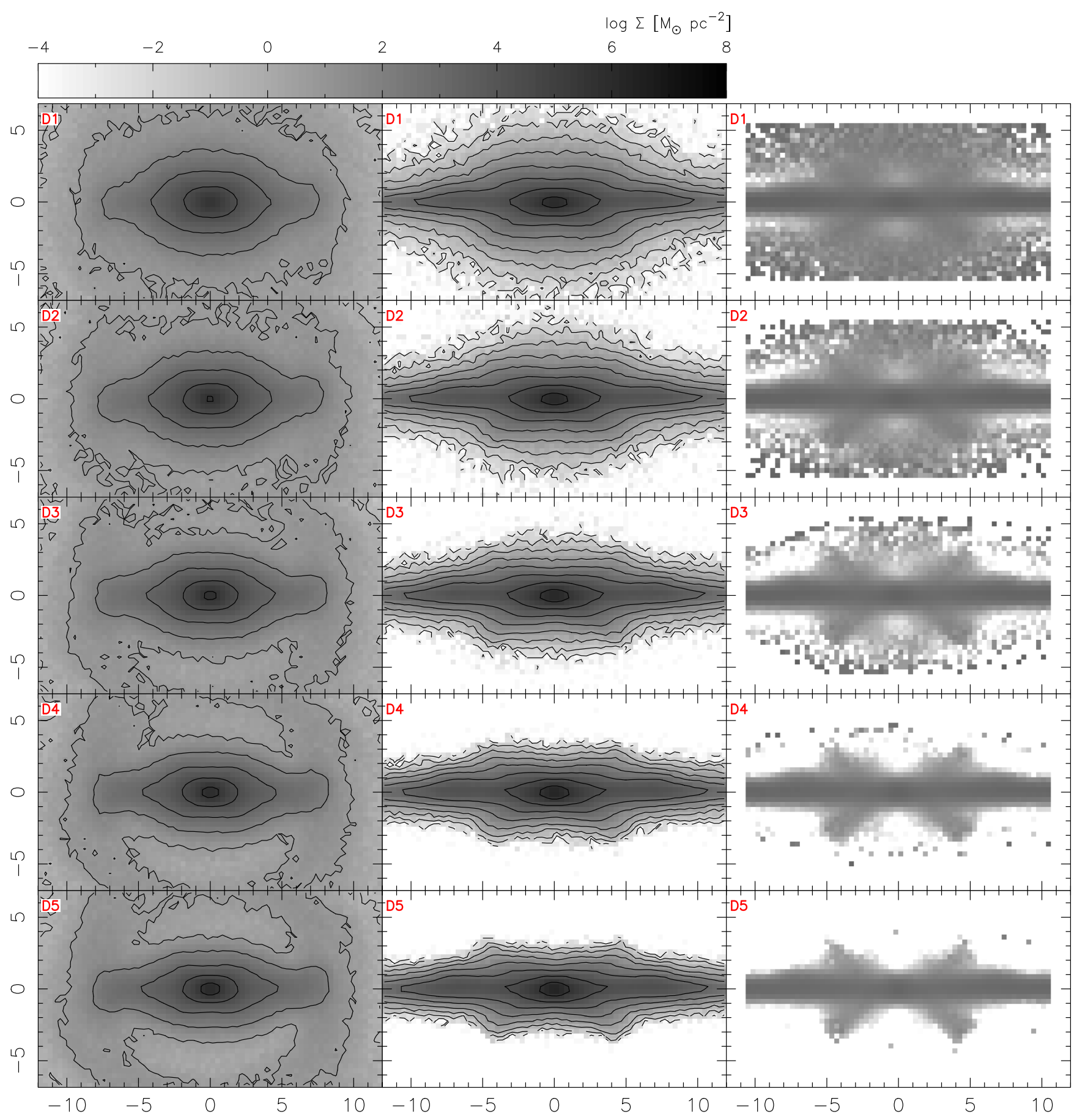

Figure 4. The face-on (left-hand panels) and edge-on (middle panels) surface density of stars in each disc population of model CU at 5 Gyr. These two columns share a common density scale indicated by the wedge at top. The right-hand panels show an unsharp mask of the edge-on image using a square kernel of width $1.4 \mathrm{kpc}$. From top to bottom are shown populations D1 (initially radially hottest) to D5 (initially radially coolest). Decreasing initial $\sigma_{R}$ leads to a stronger quadrupole, a thinner disc and a more prominent peanut shape.

relative importance of the vertical and in-plane random motions to the final morphology.

\section{SIMULATION WITH STAR FORMATION}

Having explored the effect of different in-plane kinematics with pure $\mathrm{N}$-body simulations, we now examine a simulation in which the stars form continuously out of gas, allowing the ages and random motions (in-plane and vertically), as well as the chemical properties, to be correlated. We consider the evolution of the model presented by Cole et al. (2014) and Ness et al. (2014). Briefly, in this simulation, a disc galaxy forms entirely out of gas cooling from a spherical corona and settling into a disc, triggering continuous star formation. This star-forming simulation, which was evolved with GASOLINE (Wadsley, Stadel \& Quinn 2004), the smooth particle hydrodynamics version of PKDGRAV, is extremely useful for deciphering the evolution of 


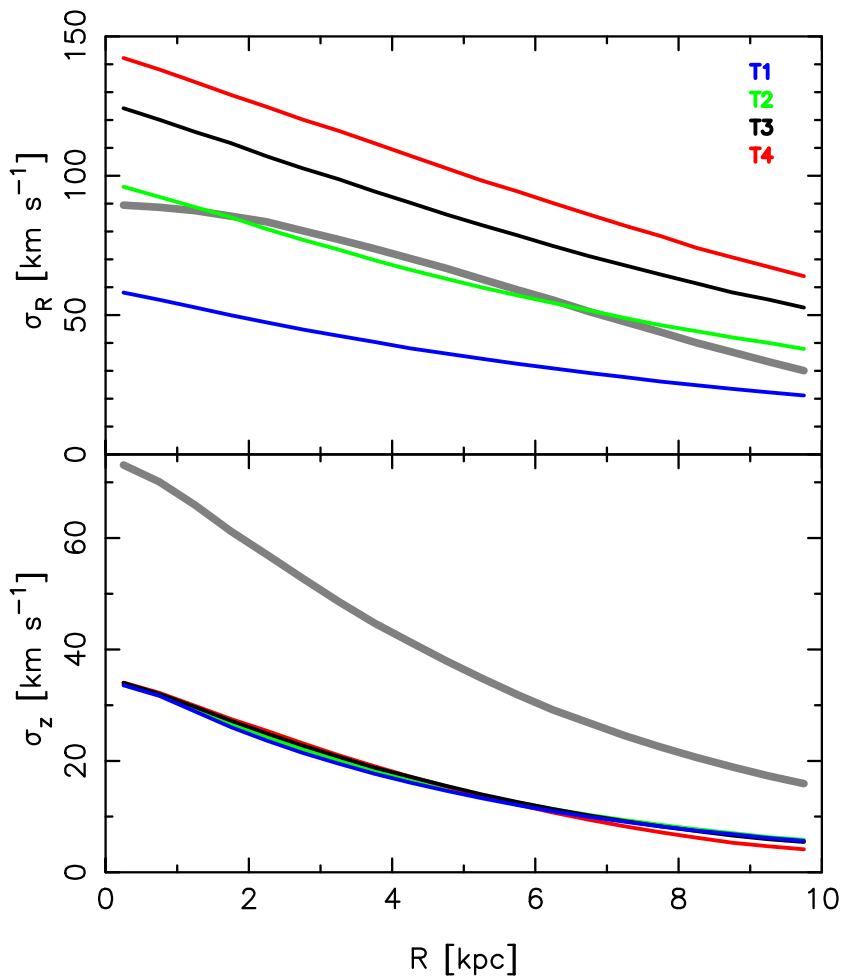

Figure 5. The initial conditions for the thinner+thicker discs simulations. The top panel shows $\sigma_{R}$, while the bottom panel shows $\sigma_{z}$. In both panels, the thick grey line shows the thicker disc, which is common to all four models, while the different thinner discs, T1-T4, are shown by the coloured lines as indicated in the top panel. In model $\mathrm{T} 2$, the $\sigma_{R}$ profile of the thinner disc (green line) is set to approximately match that of the thicker disc (grey line).

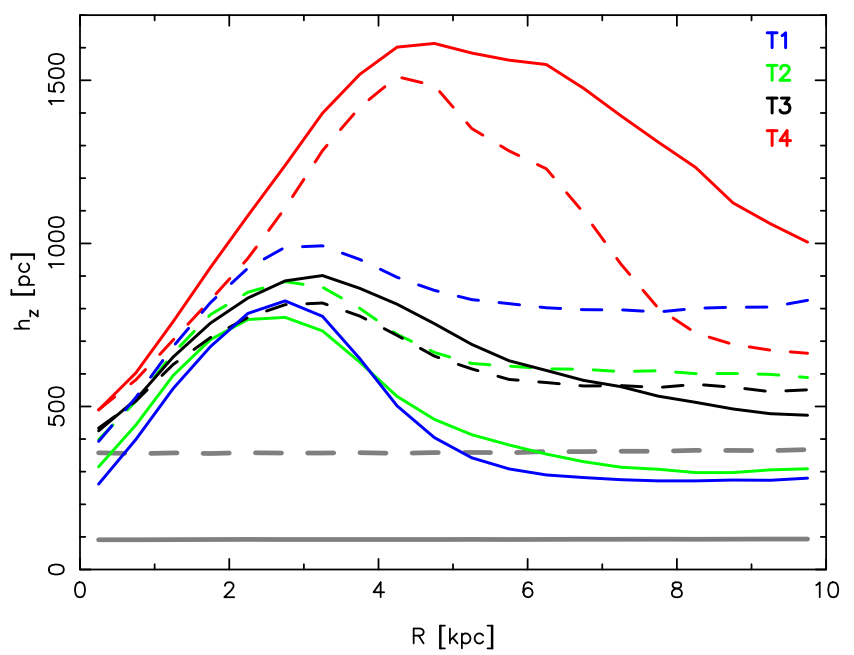

Figure 6. Initial and final height profiles for the thinner+thicker discs simulations. The horizontal grey lines correspond to the initial conditions, with the solid line showing the thinner disc and the dashed line showing the thicker disc. The remaining lines show the height profiles after evolving for 5 Gyr. Note the inversion in final thickness, with the thinner discs attaining larger heights than the thicker discs, in models T3 and, especially, T4.

the Milky Way's bulge for a number of reasons. It has high resolution (50 pc spatially and with a stellar mass of $9.5 \times 10^{3} \mathrm{M}_{\odot}$ ), forms a strong bar, and all stars form from gas, rather than put in by hand as in most previous simulation models of the Milky Way (e.g. Sell-

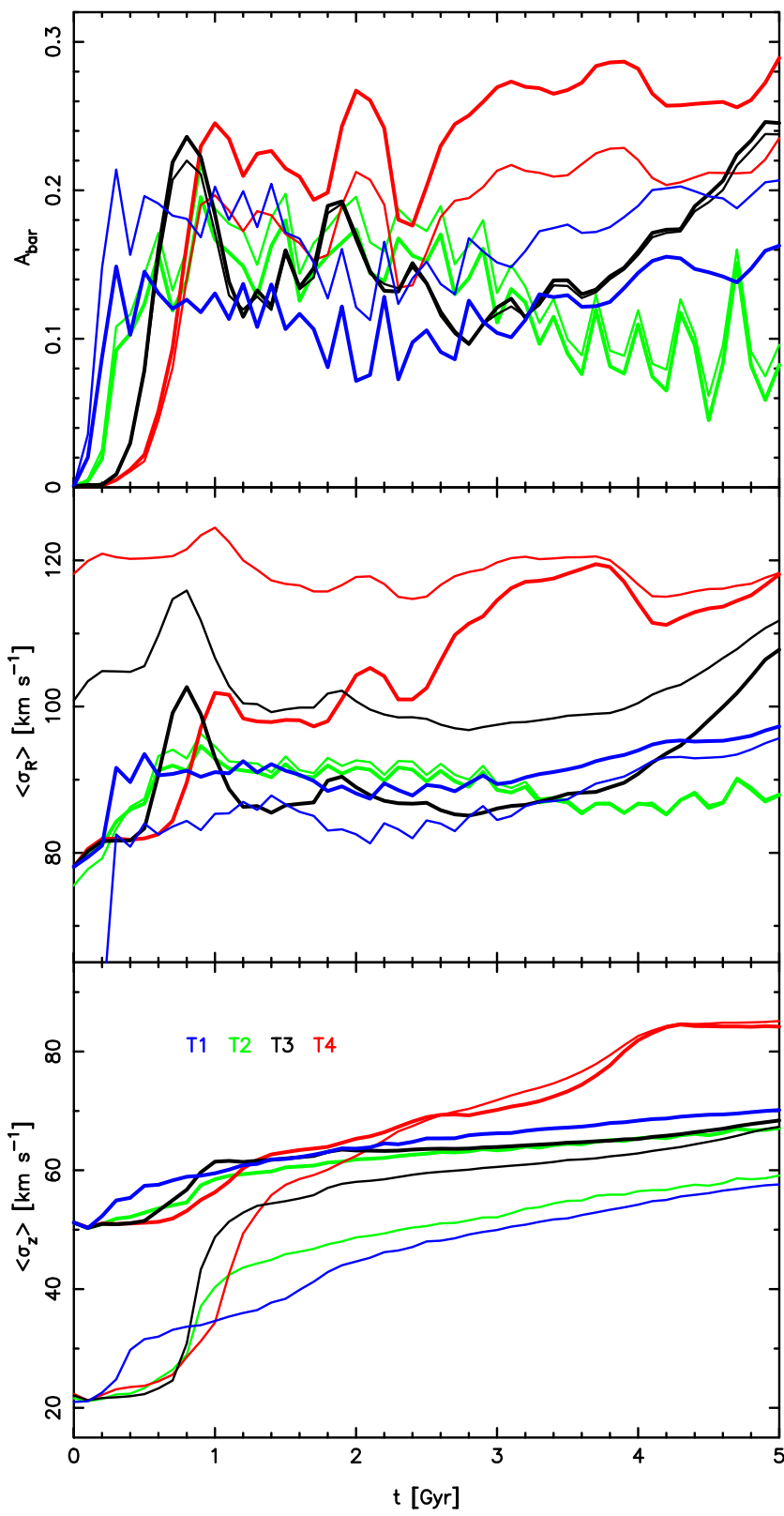

Figure 7. Evolution of the thinner+thicker discs simulations. From top to bottom, we plot the bar amplitude, $\left\langle\sigma_{R}\right\rangle$, and $\left\langle\sigma_{z}\right\rangle$. The thin lines show the thinner discs with the thick lines showing the thicker discs. Based on Fig. 6, we average the profiles in the radial range $1 \leq R / \mathrm{kpc}<5$, to match the region of peak $h_{z}$. Note the change in the relative strength of the bars in the thinner and thicker discs between models $\mathrm{T} 1$ and $\mathrm{T} 4$, and the strong vertical heating in model $\mathrm{T} 4$.

wood 1985; Fux 1997, 1999; Shen et al. 2010; Martinez-Valpuesta \& Gerhard 2011; Saha, Martinez-Valpuesta \& Gerhard 2012; Ness et al. 2013b; Gardner et al. 2014). Other studies (e.g. Samland 2002; Brook et al. 2004; House et al. 2011; Guedes et al. 2013; Bird et al. 2013) have considered the evolution of various aspects of the Milky Way via simulations in which the disc forms from gas, but the bar and bulge have received comparatively less attention in such work. The simulation tracks the dispersal of iron and oxygen, using the yields of Raiteri, Villata \& Navarro (1996, SN II), Thielemann, Nomoto \& Yokoi (1986, SN Ia) and Weidemann (1987, stellar 


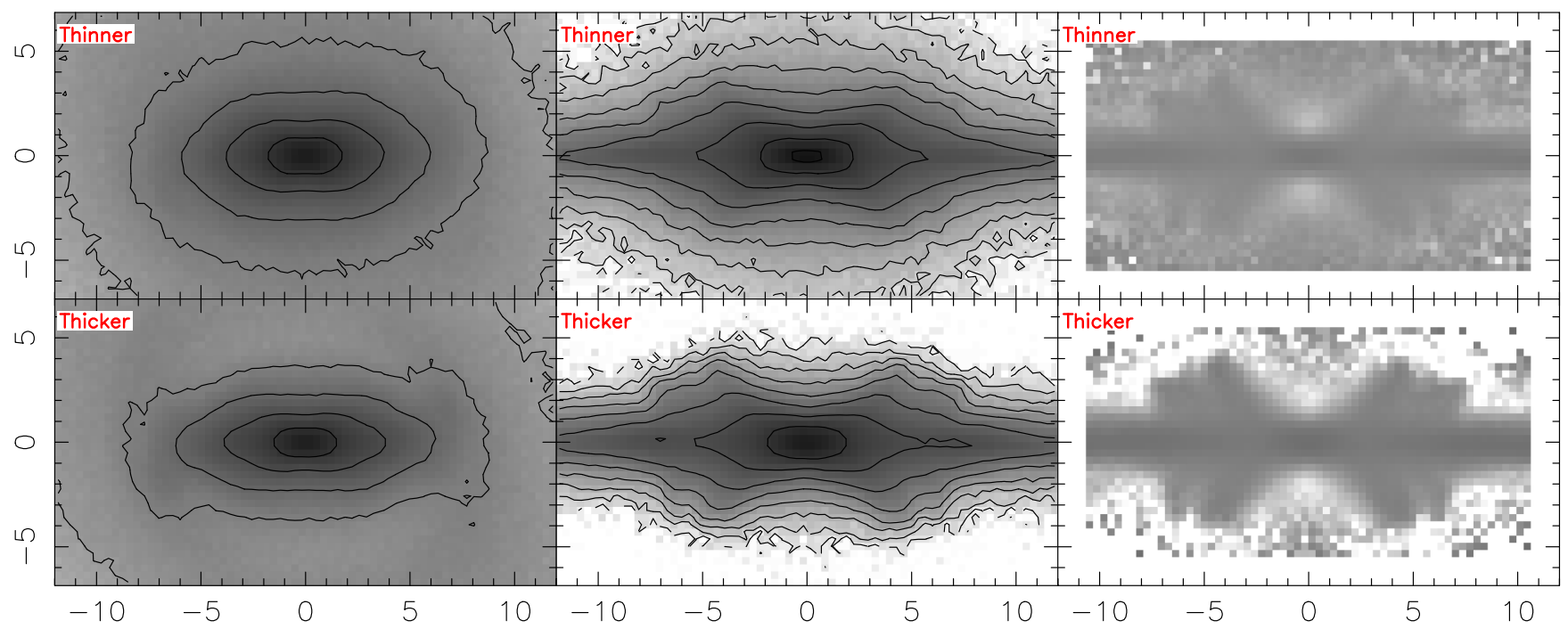

Figure 8. The face-on (left-hand panels) and edge-on (middle panels) surface density of stars in the thinner (top row) and thicker (bottom row) disc populations of model T4 at 5 Gyr. These two columns share a common density scale with the first two columns of Fig. 4. The right-hand panels show an unsharp mask of the edge-on image using a square kernel of width $1.4 \mathrm{kpc}$. The initially thinner disc ends thicker, with a less peanut-shaped bulge, than the initially thicker disc, demonstrating that the main driver of the final morphology is not the thickness of the initial disc, but its in-plane random motion.

winds), allowing us to dissect the model not just by age, but also by metallicity, $[\mathrm{Fe} / \mathrm{H}]$, and $\alpha$-enhancement, $[\mathrm{O} / \mathrm{Fe}]$.

Cole et al. (2014) showed that the model forms between 2 and 4 Gyr, slowly growing longer after then (their figs 1 and 2). By $10 \mathrm{Gyr}$, a B/P-shaped bulge has formed as can be seen in fig. 2 of Ness et al. (2014). Because we are interested in comparing the model to the Milky Way, we scale the model in size and velocity to approximate the size of the bar and the bulge rotational velocity as seen from the Sun, as described in Ness et al. (2014, multiplying coordinates by 1.2 and velocities by 0.48 ). We use the same scalefactors at all times when presenting this simulation.

\subsection{The system before bar formation}

At $2 \mathrm{Gyr}$, the model has already formed 55 per cent of the total final stellar mass, which is not uncommon for Sb-type galaxies (e.g. Kennicutt, Tamblyn \& Congdon 1994; Tacchella et al. 2015), although on average Milky Way mass galaxies reached half their mass at $z \sim 1-1.4$ (Behroozi, Wechsler \& Conroy 2013; Patel et al. 2013; Terrazas et al. 2016; van Dokkum et al. 2013). We measure the properties of the stellar distribution at this time as a function of the time of formation, $\tau$, of the stars. We separate stars in $0.5 \mathrm{Gyr}$ bins in $\tau$ and from here on refer to each such bin as a separate population.

We emphasize that the stars initially form in a discy distribution. Fig. 9 shows the density distribution of the oldest stellar population, with $0.0 \leq \tau \leq 0.5 \mathrm{Gyr}$, at $t=0.5 \mathrm{Gyr}$, i.e. just after formation. Strong spirals can be seen, as can a few very weak star-forming clumps. At $t=10 \mathrm{Gyr}$, this population still retains a discy character, although the stars have spread out, both radially and vertically, considerably.

Fig. 10 shows the average radial, $\left\langle\sigma_{R}\right\rangle$, and average vertical, $\left\langle\sigma_{z}\right\rangle$, velocity dispersions, for the stars born before $2 \mathrm{Gyr}$. At $2 \mathrm{Gyr}$, the older stellar populations are radially and vertically hotter than the younger populations. The top row of Fig. 11 maps the average metallicity, $\langle[\mathrm{Fe} / \mathrm{H}]\rangle$, at $t=2 \mathrm{Gyr}$. $\langle[\mathrm{Fe} / \mathrm{H}]\rangle$ rises rapidly in the first 2 Gyr. For all populations, the central $\sim 500 \mathrm{pc}$ is more metal-rich with a small $[\mathrm{Fe} / \mathrm{H}]$ gradient outside this region. A smallscale $(\sim 0.5 \mathrm{kpc})$ bar is evident in the mass distribution, which pro-

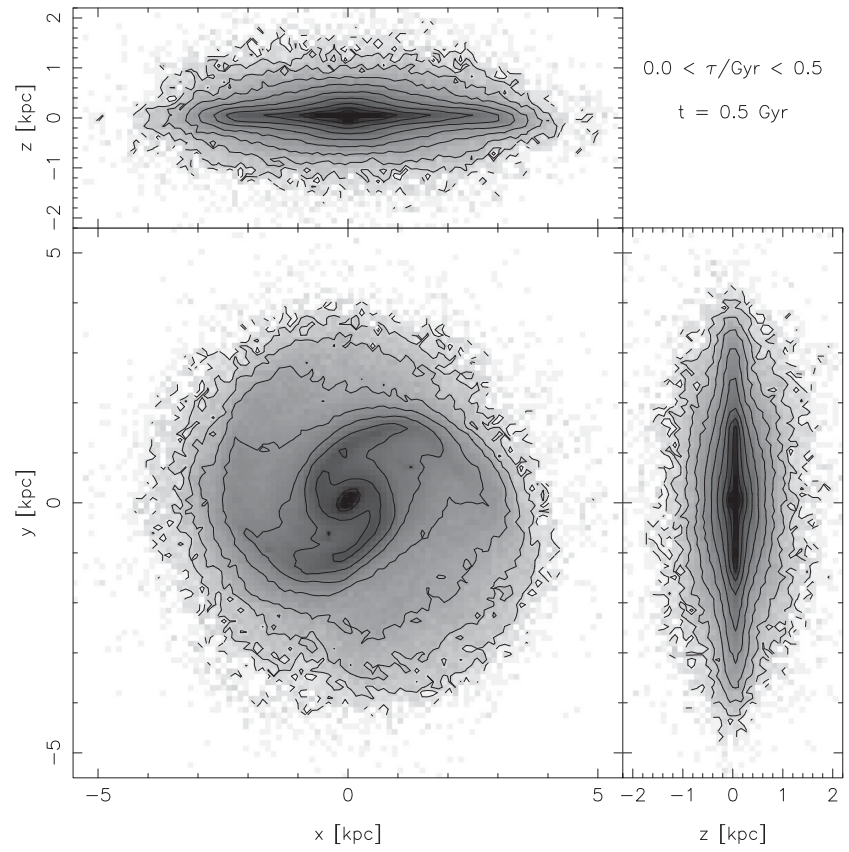

Figure 9. The oldest stellar population $(0.0 \leq \tau \leq 0.5 \mathrm{Gyr})$ at $t=0.5 \mathrm{Gyr}$ in the star-forming simulation. The distribution of these stars is discy and thin at formation, not a thick spheroid.

vides the seed around which the larger bar grows. We also examined maps of the average $\alpha$-abundance, $\langle[\mathrm{O} / \mathrm{Fe}]\rangle$. These show that $\langle[\mathrm{O} / \mathrm{Fe}]\rangle$ drops rapidly with increasing $\tau$.

Thus overall, at $2 \mathrm{Gyr}$, before the bar has formed, the age of a stellar population correlates with $\left\langle\sigma_{R}\right\rangle$, and $\langle[\mathrm{O} / \mathrm{Fe}]\rangle$, and anticorrelates with $\langle[\mathrm{Fe} / \mathrm{H}]\rangle$. These dependencies on age imply that $\sigma_{R}$ correlates with chemistry, which we show explicitly in Fig. 12.

Therefore, on the basis of the pure $N$-body simulations, we expect that the bar will cause the different populations to separate morphologically, forming a stronger bar in the younger, metal-rich 


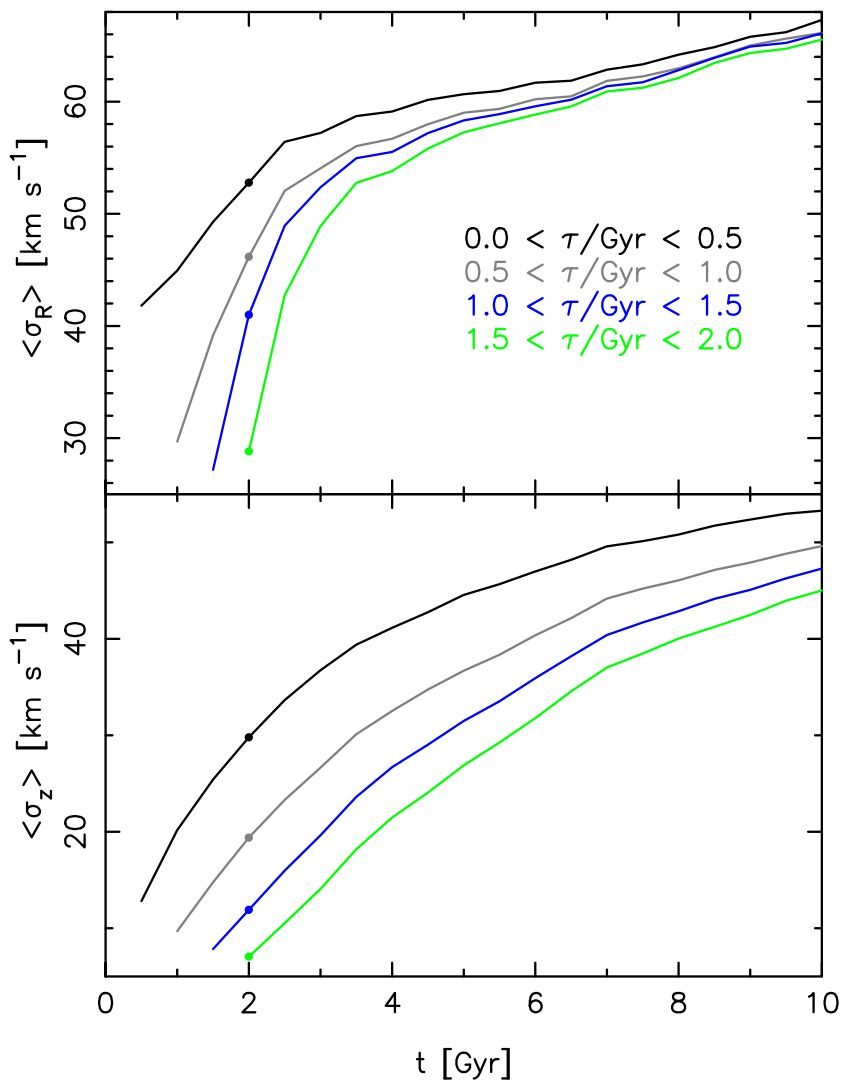

Figure 10. The evolution of $\left\langle\sigma_{R}\right\rangle$ (top), and $\left\langle\sigma_{z}\right\rangle$ (bottom) in the starforming simulation. The different $\tau$ populations are indicated in the top panel. Averages are taken over the radial range $1.5 \leq R / \mathrm{kpc} \leq 3$. Note that at $2 \mathrm{Gyr}$ (marked by the filled circles), as the bar starts forming, the radial and vertical dispersions increase with $\tau$.

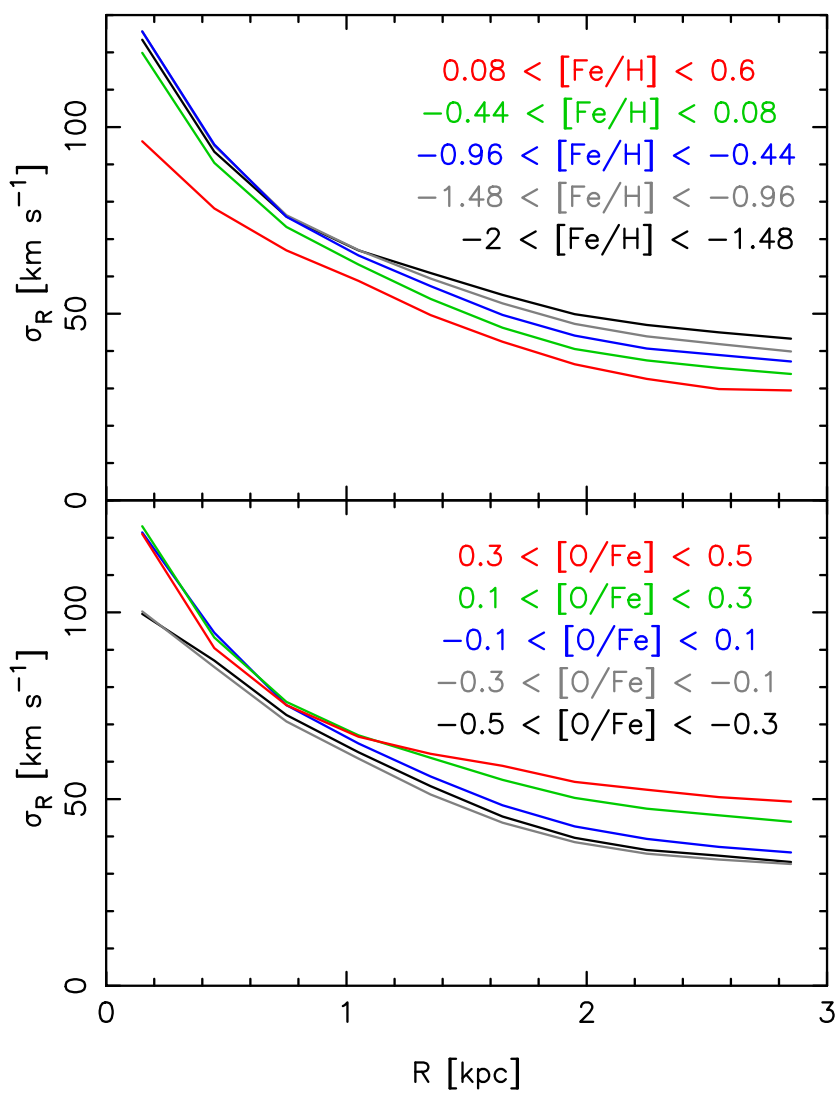

Figure 12. The (anti)correlation between $([\mathrm{Fe} / \mathrm{H}])[\mathrm{O} / \mathrm{Fe}]$ and $\sigma_{R}$ at $t=2 \mathrm{Gyr}$, before the bar has formed in the star-forming simulation. These relations arise because $\sigma_{R},[\mathrm{Fe} / \mathrm{H}]$ and $[\mathrm{O} / \mathrm{Fe}]$ all evolve with time.
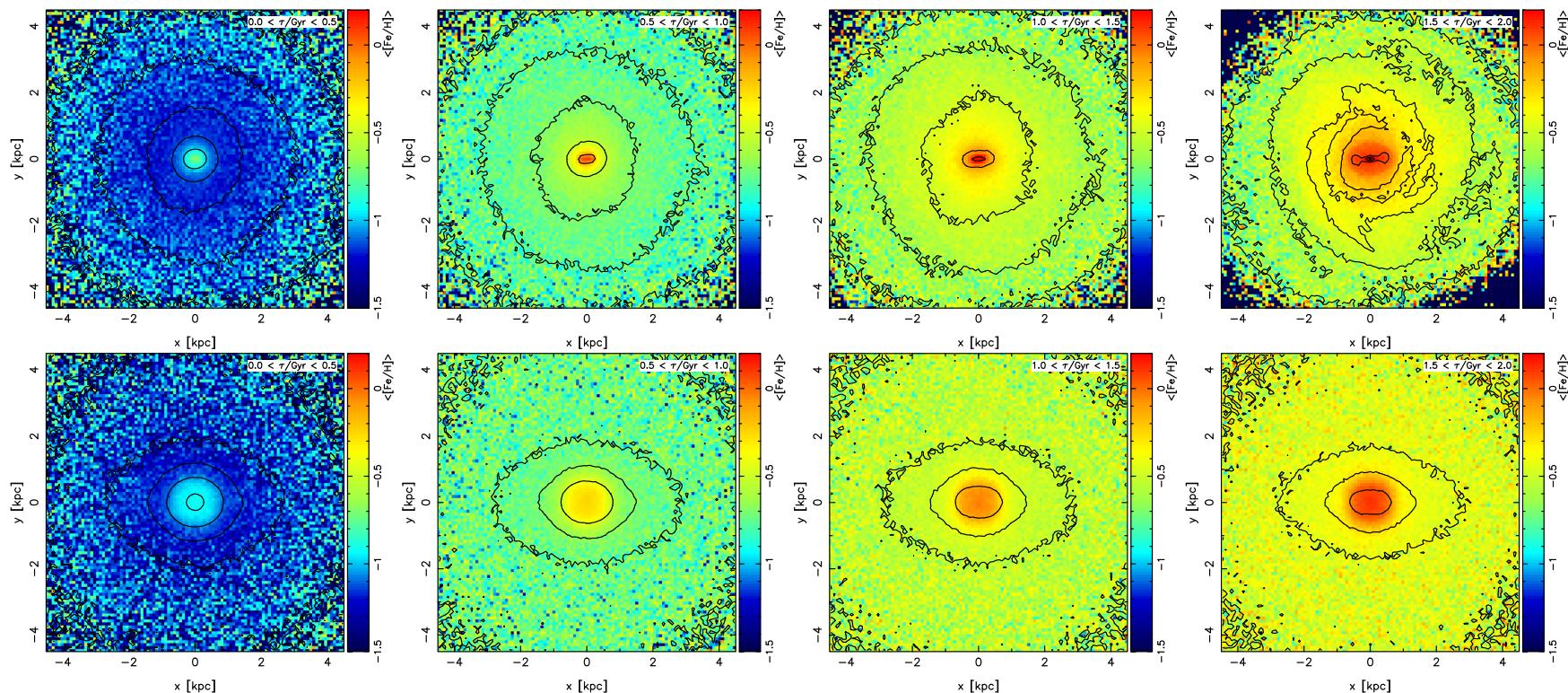

Figure 11. Maps of $\langle[\mathrm{Fe} / \mathrm{H}]\rangle$ at $t=2 \mathrm{Gyr}$ (top row) and at $t=10 \mathrm{Gyr}$ (bottom row) for different $\tau$ populations as indicated at the top right of each map (from left to right, $0.0 \leq \tau / \mathrm{Gyr} \leq 0.5,0.5 \leq \tau / \mathrm{Gyr} \leq 1.0,1.0 \leq \tau / \mathrm{Gyr} \leq 1.5$ and $1.5 \leq \tau / \mathrm{Gyr} \leq 2.0$ ), in the star-forming simulation. The colour scale, indicated by the wedge beside each map, is common to all panels and spans $-1.5 \leq[\mathrm{Fe} / \mathrm{H}] \leq 0.2$. Contours indicate the surface density of the different populations . The metallicity of populations rises rapidly with $\tau$. 


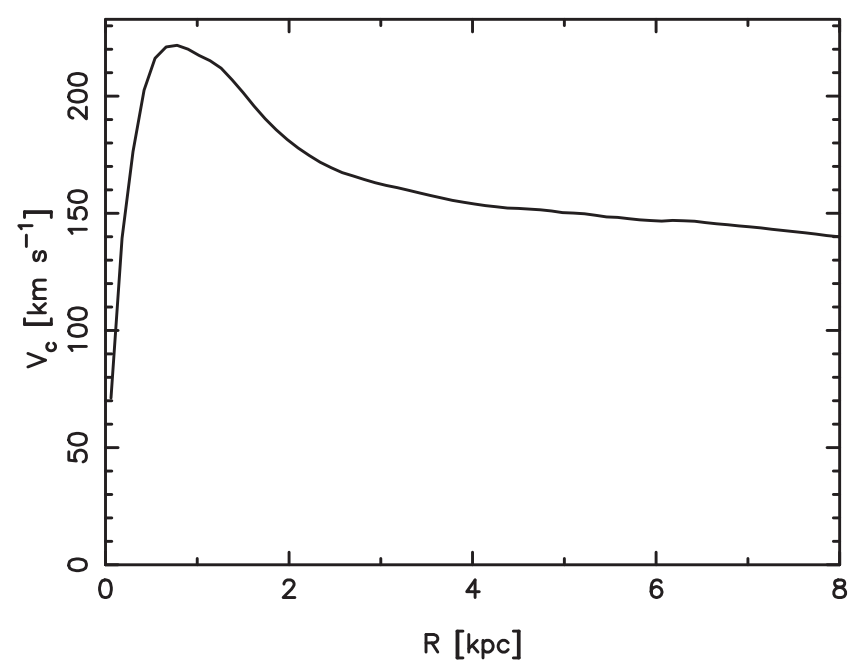

Figure 13. The azimuthally averaged rotation curve of the star-forming simulation after $10 \mathrm{Gyr}$.

populations and a more ellipsoidal distribution in the older, metalpoor populations. As with $\sigma_{R}, \sigma_{z}$ also correlates with age, as expected since dispersions increase continuously at this time. Thus, $\sigma_{z}$ will also correlate with chemistry, but the driver of the separation of populations will be $\sigma_{R}$, not $\sigma_{z}$, as we showed in Section 3.2 above for the pure $N$-body simulations.

\subsection{The final system}

The azimuthally averaged rotation curve of this model at $10 \mathrm{Gyr}$ is shown in Fig. 13. This exhibits a central peak which then drops off and flattens. Because we are studying the bulge of the model, our velocity scaling is determined by comparing the bulge with that of the Milky Way, which has a rather flatter rotation curve (e.g. Bland-Hawthorn \& Gerhard 2016; Li et al. 2016). Further out, the rotation velocity of our model is lower than that of the Milky Way.

\subsubsection{Velocity dispersions}

Fig. 10 shows the evolution of $\left\langle\sigma_{R}\right\rangle$ and $\left\langle\sigma_{z}\right\rangle$. We average in the range $1.5 \leq R / \mathrm{kpc} \leq 3$ in order to avoid the effects of the nuclear disc (Cole et al. 2014; Debattista et al. 2015). The formation of the bar raises $\left\langle\sigma_{R}\right\rangle$ sharply, while $\left\langle\sigma_{z}\right\rangle$ increases more slowly. As in the pure $N$-body simulations, $\left\langle\sigma_{R}\right\rangle$ of the different populations evolve towards each other, while their $\left\langle\sigma_{z}\right\rangle$ remain separated, with continued late time heating driven by the growing bar. While we find no single violent buckling event like in the pure $N$-body simulations, small-scale bends in the disc can be detected after $2 \mathrm{Gyr}$, which contribute to the vertical heating of the inner disc.

\subsubsection{Vertical distribution}

The top panel of Fig. 14 plots profiles of $h_{z}$ for the different $\tau$ populations. All populations thicken considerably outside $R=500 \mathrm{pc}$ between 2 and $10 \mathrm{Gyr}$, with the height of the oldest population roughly doubling at $R=2 \mathrm{kpc}$. For stars with $2 \leq \tau / \mathrm{Gyr} \leq 4$, the profile is locally peaked at $R \simeq 1.6 \mathrm{kpc}$ rather than monotonically increasing, indicating that these younger populations are peanutshaped.

The bottom panel of Fig. 14 plots the vertical density profiles along the minor axis of the disc at $10 \mathrm{Gyr}$ for different $\tau$ populations,
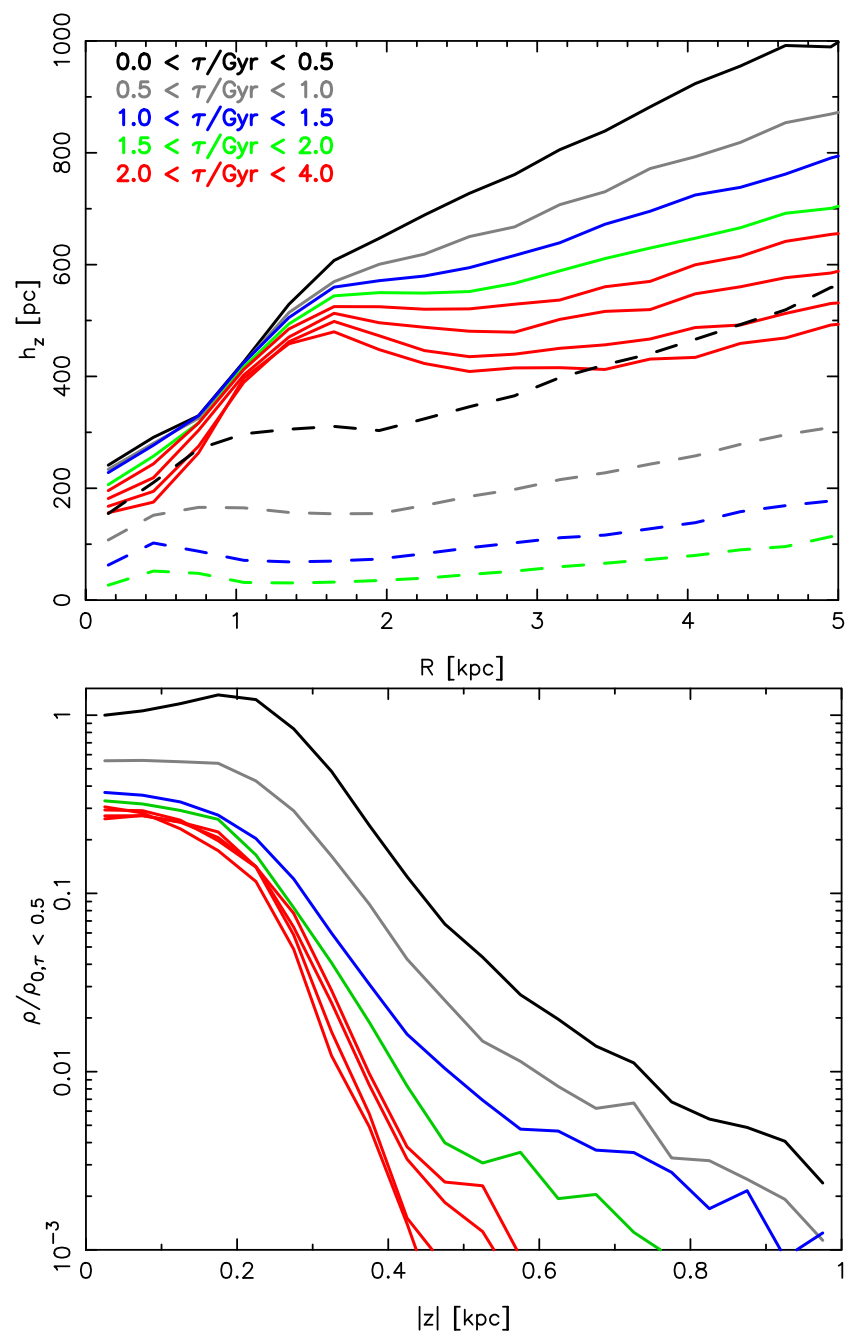

Figure 14. Top: vertical height of stars at $t=2 \mathrm{Gyr}$ (dashed lines) and at $10 \mathrm{Gyr}$ (solid lines) for the different $\tau$ populations in the star-forming simulation. The younger stars have a prominent local peak at $R \sim 1.7 \mathrm{kpc}$, coinciding with their stronger peanut shape. Bottom: vertical density profile along the minor axis of the disc at $10 \mathrm{Gyr}$. The profiles are normalized to the central density of the oldest population. Colours are as in the top panel. The red lines show four bins, of equal width in $\tau$, of stars born at $2 \leq \tau / \mathrm{Gyr} \leq 4$. The younger populations extend to lower height than the older populations.

normalized by the mid-plane density of the oldest population. The oldest population is clearly the one that extends the furthest in height and we have verified that still younger populations reach even lower height. The density of the oldest population peaks at $z \simeq 200 \mathrm{pc}$ rather than at the mid-plane. This may be related to the boxy-core structure reported by Li \& Shen (2015).

\subsubsection{Bar strength}

The left-hand panels of Fig. 15 show the final projected stellar surface density in different populations. There is a clear trend for the stellar distribution to become more strongly barred the later the stars formed. For stars formed before the bar ( $\tau \leq 2 \mathrm{Gyr})$, there is a strong dependence of bar strength on age. The bottom panel of Fig. 16 shows the evolution of bar amplitude, $A_{\text {bar }}$, as a function of $\tau$. Populations with $\tau<1.5 \mathrm{Gyr}$ end up in a weaker bar compared with populations that form later. The various populations with $2 \leq$ $\tau /$ Gyr $\leq 4$ have very similar global amplitude. The bar amplitude 

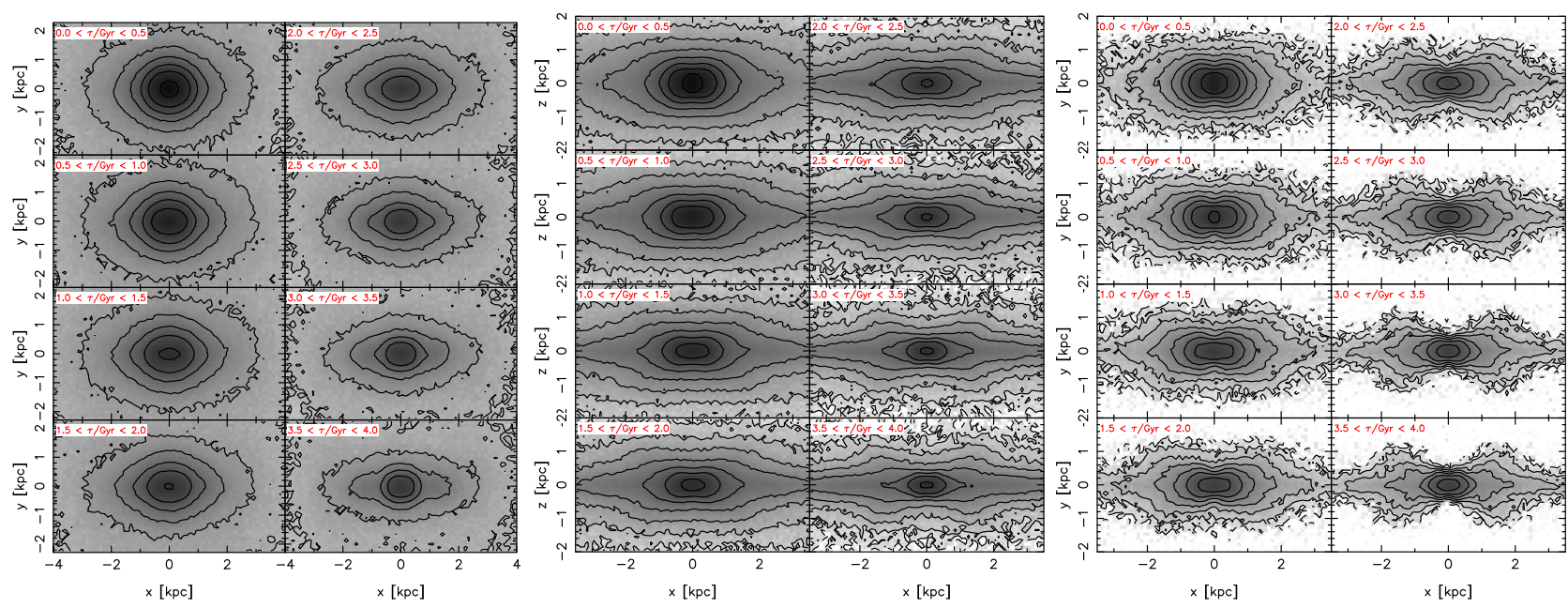

Figure 15. The face-on (left two columns) and edge-on (middle two columns) surface density distribution for different $\tau$ populations, as indicated, in the star-forming simulation at $t=10 \mathrm{Gyr}$. The right two columns show a cross-section with $|y| \leq 0.2 \mathrm{kpc}$ through the edge-on views of the middle panels. In the edge-on panels, the bar is perpendicular to the line of sight (i.e. it is viewed side-on). Note that slightly different spatial scales are used for the face-on and the edge-on views. The quadrupole moment and peanut shape both increase rapidly with $\tau$.
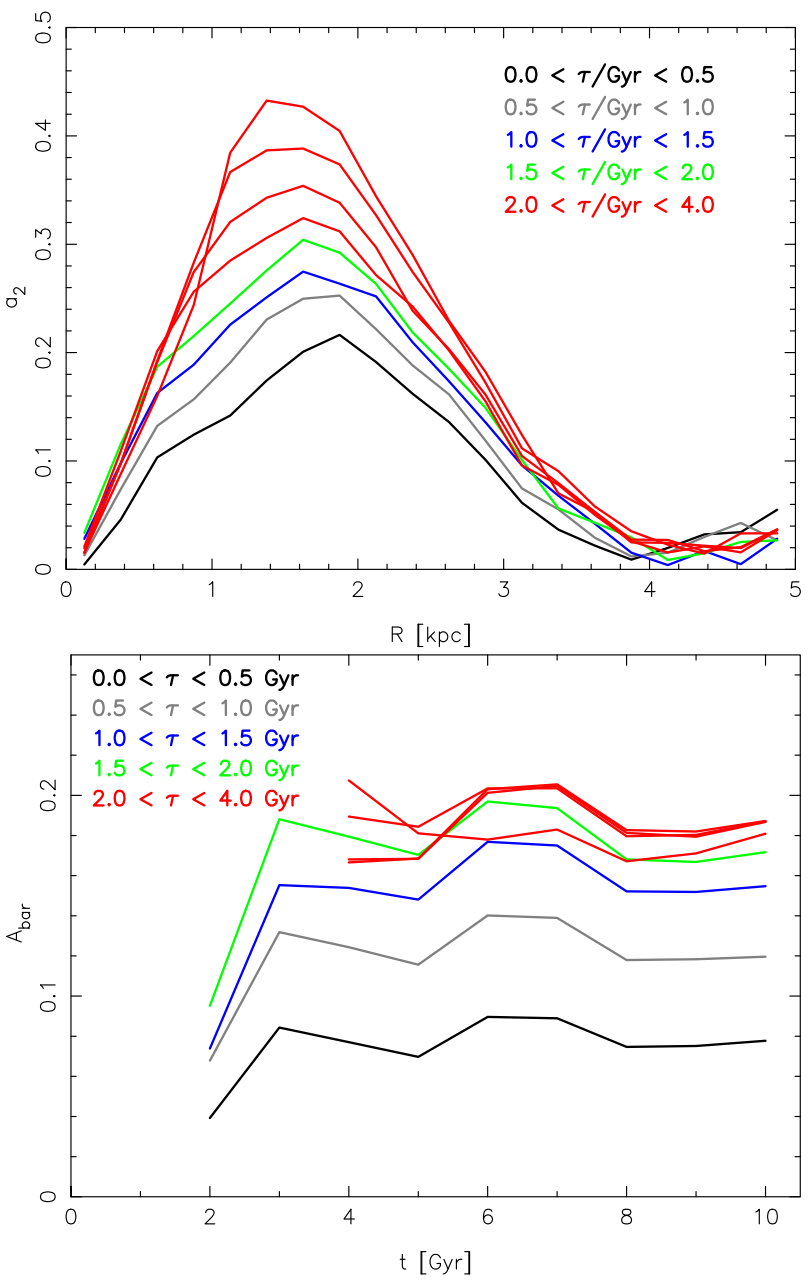

Figure 16. Top: final radial $m=2$ amplitude, $a_{2}(m=2$ Fourier amplitude in radial bins), of the surface density of stars in different $\tau$ populations in the star-forming simulation. Bottom: the evolution of bar amplitude, $A_{\text {bar }}$, for different $\tau$ populations, as indicated. Globally and at each radius, the bar is weakest in the oldest stellar populations. The red lines show four bins, of equal width in $\tau$, of stars born at $2 \leq \tau / \mathrm{Gyr} \leq 4$ of the old populations evolves in parallel with that of the younger, cooler populations without ever becoming as strong.

The top panel of Fig. 16 presents the radial profile of the $m=2$ Fourier moment, $a_{2}$, showing that the peak amplitude decreases with age. Populations formed during $2 \leq \tau / \mathrm{Gyr} \leq 4$ also have different profiles, in spite of their equal overall bar strength, with peak amplitude increasing with $\tau$. In Fig. 15, this is directly evident as an increasingly elongated density distribution. None the less the bar has the same semimajor axis size for all populations.

\subsubsection{Peanut shape}

The middle and right-hand panels of Fig. 15 show the edge-on distribution of different $\tau$ populations, with the bar viewed side-on. As $\tau$ increases, the disc becomes thinner, particularly at the centre and the shape changes from boxy to peanuty, in agreement with the radial profiles of $h_{z}$ shown in the top panel of Fig. 14. The very oldest populations have a thick, boxy spheroidal shape. The younger populations are thinner at the centre, resulting in a peanut structure. As with the bar strength, this result is in agreement with the pure $N$-body simulations.

\subsubsection{Chemistry}

The bottom row of Fig. 11 shows maps of $\langle[\mathrm{Fe} / \mathrm{H}]\rangle$ at $t=10 \mathrm{Gyr}$, which can be compared with the maps in the top row at $t=2 \mathrm{Gyr}$. The central $\langle[\mathrm{Fe} / \mathrm{H}]\rangle$ remains high, although it is distributed over a slightly larger region than at $2 \mathrm{Gyr}$. Other $\langle[\mathrm{Fe} / \mathrm{H}]\rangle$ gradients within each $\tau$ population are largely erased, evidence of the significant mixing over this region.

The top panel of Fig. 17 shows the vertical $\langle[\mathrm{Fe} / \mathrm{H}]\rangle$ profiles on the bar's minor axis. A non-zero gradient is present which at large $|z|$ is dominated by the oldest population. The vertical $\langle[\mathrm{Fe} / \mathrm{H}]\rangle$ gradient for all stars varies with $|z|$, becoming flatter with height. The bottom panel shows similar profiles for $\langle[\mathrm{O} / \mathrm{Fe}]\rangle$. The oldest population is very $\alpha$-enhanced, dropping rapidly with $\tau$. The $\langle[\mathrm{O} / \mathrm{Fe}]\rangle$ of all stars is low near the mid-plane where stars are still forming but is quickly dominated by the oldest stellar population by $\sim 400 \mathrm{pc}$. In both panels, the profile within each $\tau$ bin is flatter than the overall 


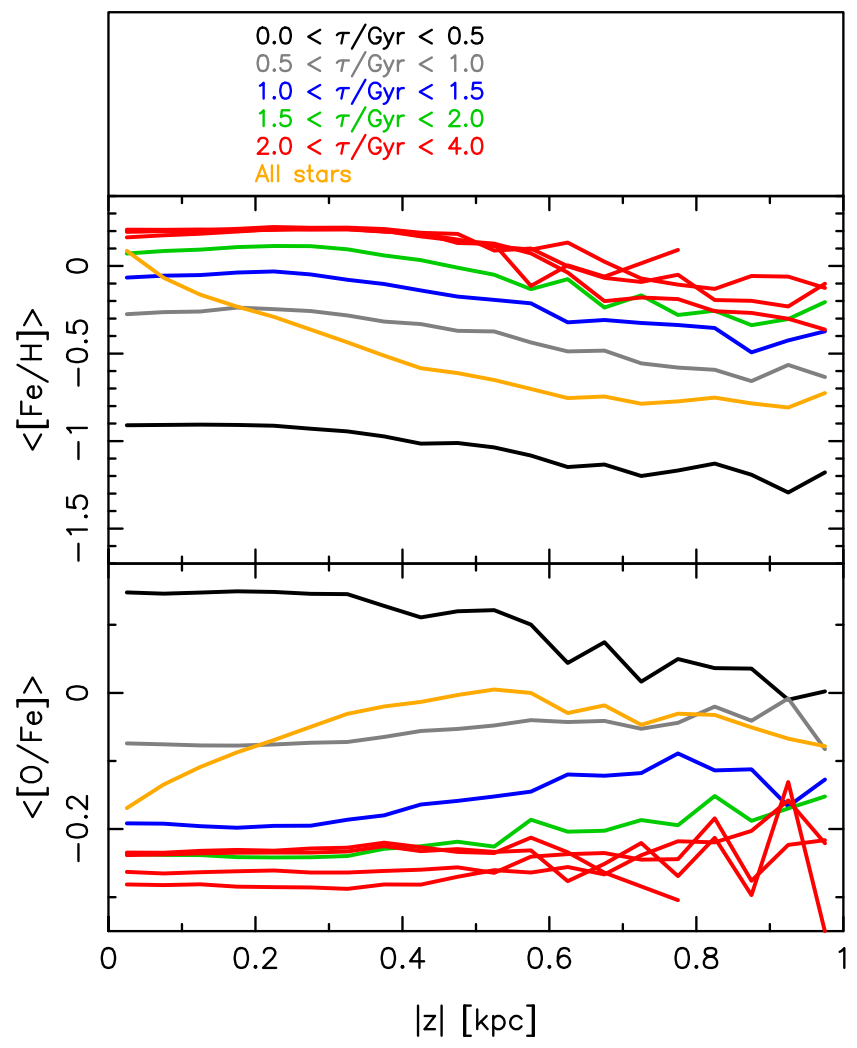

Figure 17. Vertical profiles of stellar chemistry on the minor axis of the bar (with $R<0.3 \mathrm{kpc}$ ) at $10 \mathrm{Gyr}$ for different populations in the star-forming simulation. The top panel shows $\langle[\mathrm{Fe} / \mathrm{H}]\rangle$, while the bottom one shows $\langle[\mathrm{O} / \mathrm{Fe}]\rangle$. The total profiles are indicated by the orange lines. The strong gradients are largely due to the different vertical extent of different age populations, not to internal gradients within each $\tau$ population, which are quite shallow. The red lines show four bins, of equal width in $\tau$, of stars born at $2 \leq \tau / \mathrm{Gyr} \leq 4$.

profile, indicating that the overall steep gradients in $\langle[\mathrm{Fe} / \mathrm{H}]\rangle$ and $\langle[\mathrm{O} / \mathrm{Fe}]\rangle$ are produced by variations in the relative densities of different age populations. Once the oldest population dominates the vertical profile, the chemical gradients become much flatter.

\subsubsection{Dependence of height on metallicity}

The top panel of Fig. 18 plots the height profiles of stars for different bins in $[\mathrm{Fe} / \mathrm{H}]$. The metal-rich stars comprise a thinner distribution than do the metal-poor ones. A peak is present at $R \simeq 1.6 \mathrm{kpc}$ in the high metallicity $h_{z}$ profile, whereas it rises monotonically for the more metal-poor stars. The peak in the metal-rich stars is a signature of the peanut-shaped distribution in the younger populations, and the absence of a peak in the lowest $[\mathrm{Fe} / \mathrm{H}]$ stars is consistent with the absence of a peanut shape in old stars. The height profiles for different $[\mathrm{O} / \mathrm{Fe}]$ bins are qualitatively similar, with the $[\mathrm{O} / \mathrm{Fe}]-$ rich population monotonically increasing in height, whereas the $[\mathrm{O} / \mathrm{Fe}]$-poor population exhibits a local peak. Bearing in mind the anticorrelation between age and $[\mathrm{Fe} / \mathrm{H}]$, and the correlation with [O/Fe], Figs 14 and 18 tell a consistent story.

\subsection{Synthesis of the pure $N$-body and star-forming simulations}

We have shown that the evolution of both the kinematics and the morphology of the star-forming simulation are consistent with those

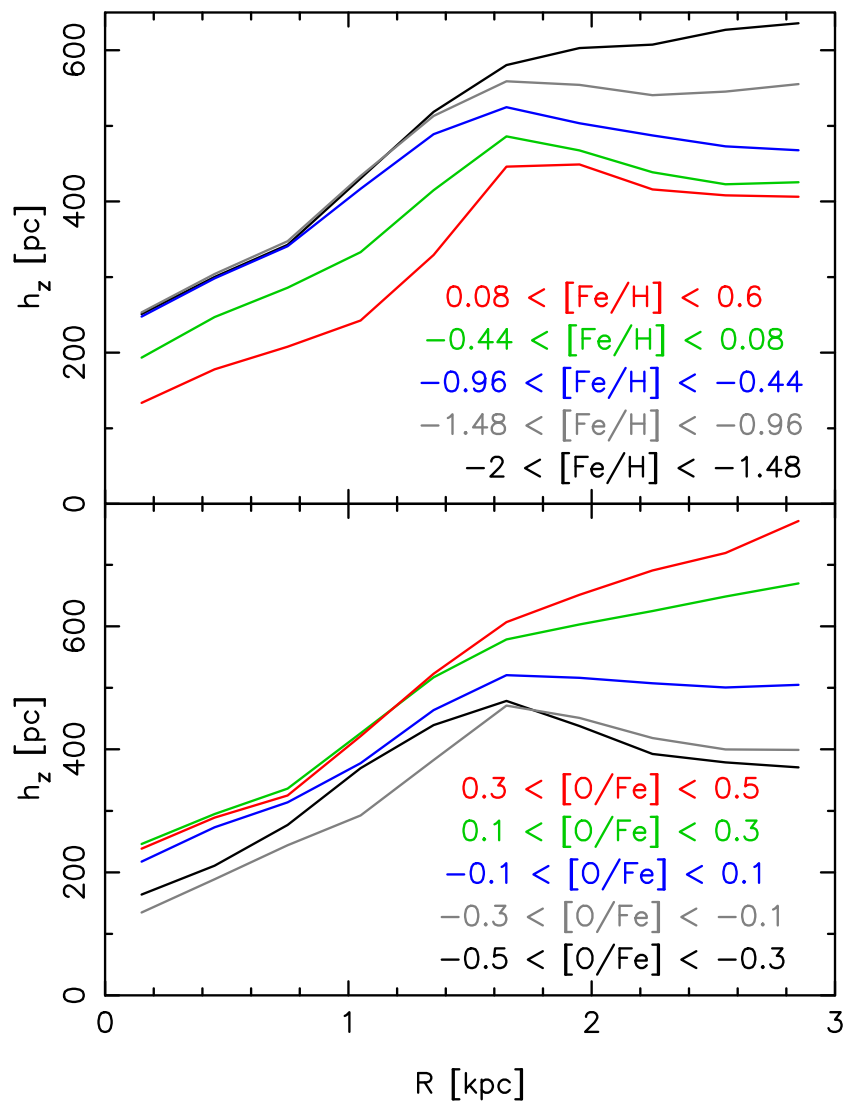

Figure 18. Profiles of the vertical height for different $[\mathrm{Fe} / \mathrm{H}]$ (top) and $[\mathrm{O} / \mathrm{Fe}]$ bins (bottom) at $10 \mathrm{Gyr}$ in the star-forming simulation. Peaks, associated with a peanut, rather than boxy, shape, are evident at high $[\mathrm{Fe} / \mathrm{H}]$ and low $[\mathrm{O} / \mathrm{Fe}]$.

of the pure $N$-body simulations: populations with smaller initial in-plane velocity dispersions end up vertically thinner with a pronounced peanut shape and a strong bar. Conversely, the hotter populations form a thicker distribution with a boxy, not peanut, shape and with a bar that has a significantly weaker quadrupole moment. The pure $N$-body simulations have, by their nature, no chemistry but if we reasonably assume that the high $\sigma_{R}$ populations are older, then we expect them to have lower $[\mathrm{Fe} / \mathrm{H}]$ and higher $[\mathrm{O} / \mathrm{Fe}]$, as in the star-forming simulation. Then, the pure $N$-body simulations would predict a strong peanut shape in the metal-rich population and a weak bar with a boxy bulge in the oldest, metal-poor population. The evolution of the bulge in the star-forming simulation can therefore be understood simply as resulting from the separation induced by differences in in-plane kinematics at bar formation.

\section{APPLICATION TO THE MILKY WAY}

Having understood how kinematic fractionation gives rise to morphological and kinematic differences between different stellar populations, we now explore whether the resulting trends can explain observations of the Milky Way's bulge by comparing the star formation model with the Milky Way. We also take this opportunity to predict trends that have not been observed before. We scale the model in the same way as above and view the model from the Sun's perspective, using the standard Galactic coordinates $(l, b)$. We assume that the Sun is $8 \mathrm{kpc}$ from the Galactic Centre, placing the observer at $y=-8 \mathrm{kpc}$, and orienting the bar at angle of $27^{\circ}$ to 
the line of sight (Wegg \& Gerhard 2013). We emphasize that in this section we are only interested in understanding trends that arise in the Milky Way's bulge, not in matching them in detail.

\subsection{Caveats about the model}

Before comparing the model with the Milky Way, it is important to mention some limitations of the model. The model is very useful for interpretating global trends, but should not be construed as a detailed model of the Milky Way, even after we rescale it. It suffers from a number of limitations which preclude efforts to test the model in a detailed quantitative way. One important difference is that the $\mathrm{X}$-shape is less prominent in the model than in the Milky Way, and rises to smaller heights. This is possibly due to the fact that the bar has not grown quite as much as in the Milky Way. The bar in the model has a radius of $\sim 3 \mathrm{kpc}$ (Cole et al. 2014), while the bar in the Milky Way extends to $\sim 4.5-5 \mathrm{kpc}$ (Wegg, Gerhard \& Portail 2015). The relatively high gas inflow rate to the centre of the model, with the attendant angular momentum transport and high star formation rate, is probably the source of this slow growth. Cole et al. (2014) showed that this gas inflow produces a nuclear disc, which Debattista et al. (2015) proposed is the origin of the high-velocity peaks seen in the mid-plane line-of-sight velocity distributions at $l \simeq 6^{\circ}-8^{\circ}$ in the APOGEE data (Nidever et al. 2012). (Alternatively Aumer \& Schönrich 2015, proposed that these high-velocity peaks are produced by young stars trapped by the bar from the disc.)

A second limitation of the model is in its chemical evolution. The simulation did not include the diffusion of metals between gas particles, without which too many low-metallicity stars form at all ages. This has the effect of broadening the metallicity distribution function and of weakening the trends between metallicity and age. With metal diffusion, a tighter correlation between age and chemistry would have resulted, making the chemical separation by the bar even stronger. Also, while the yields we use for the $[\mathrm{Fe} / \mathrm{H}]$ enrichment have been shown to lead to very good matches to the metallicity distribution functions across the Milky Way (Loebman et al. 2016), the oxygen yields may have significant offsets (e.g. Loebman et al. 2011).

Lastly, and most obviously, the simulation was only run for 10 Gyr. However by the end of the simulation, the model has long been in a phase of slow secular evolution, growing slowly while continuing to form stars.

\subsection{X-shape}

Several of the currently known morphological properties of the Milky Way bulge are based on the magnitude measurement of red clump stars. A number of studies have used bulge red clump stars from the Two Micron All-Sky Survey and the VISTA Variables in the Via Lactea (VVV) ESO public survey (Minniti et al. 2010) to derive the mean line-of-sight distances across the bulge, thus mapping its $3 \mathrm{D}$ shape. This has resulted in the discovery of an $\mathrm{X}$-shaped structure as a bimodal distance distribution (McWilliam \& Zoccali 2010; Nataf et al. 2010; Saito et al. 2011; Wegg \& Gerhard 2013; Gonzalez et al. 2015). Li \& Shen (2012) showed that a peanut-shaped bulge viewed from the Solar perspective is responsible for this X-shape. By separating red clump stars into metal-rich $([\mathrm{Fe} / \mathrm{H}]>-0.5)$ and metal-poor $([\mathrm{Fe} / \mathrm{H}]<-0.5)$ populations, Ness et al. (2012), Uttenthaler et al. (2012) and Rojas-Arriagada et al. (2014) determined that metal-poor stars do not follow the bimodal distance distribution and therefore do not trace the $\mathrm{X}$-shape of the bulge.
We now investigate the properties of the X-shape in the simulation. Gonzalez et al. (2015) have already compared the model, scaled and oriented the same way as here, with the Milky Way. They showed that the model produced a split red clump at $b=-8.5^{\circ}$ for $-2^{\circ} \leq l \leq+2^{\circ}$; at $l=+2^{\circ}$ the bright peak is more prominent, while the faint peak is more prominent at $l=-2^{\circ}$. These are the same trends as observed in the VVV survey and can be explained by an $\mathrm{X}$-shaped overdensity of stars along the line of sight.

In order to test if the model is able to reproduce the trends with metallicity, we observe the star particles assuming they are red clump stars. Because young, metal-poor stars (caused by the absence of gas metallicity mixing) contaminate the old, metal-poor bins when selecting by metallicity, in this analysis we deconstruct the $\mathrm{X}$-shape by age, rather than by $[\mathrm{Fe} / \mathrm{H}]$. The method to derive distances from red clump stars is based on the construction of the luminosity function of the bulge towards a given line of sight where the red clump can be easily identified and its mean magnitude obtained (Stanek et al. 1994). We set the absolute magnitude of stars in the model to $M_{K}=-1.61$, selecting equal numbers of stars in the magnitude bins $12.3<K<12.9$ and $12.9<K<13.58$ (chosen to sample the near and far arms of the $\mathrm{X}$-shape with equal numbers of stars, independent of the detailed density distribution, reminiscent of the ARGOS selection strategy). We convolve the magnitude distribution with a Gaussian kernel of $\sigma=0.17$ mag to simulate the intrinsic scatter of the red clump (e.g. Gerhard \& MartinezValpuesta 2012), and then transform back to distance using the distance modulus appropriate for $8 \mathrm{kpc}$.

The bottom row of Fig. 19 shows the distribution of the red clump stars in the model, at distances from the Sun $6 \leq R_{\mathrm{S}} / \mathrm{kpc} \leq 11$, for three different lines of sight on the minor axis $\left(|l|<2^{\circ}\right)$, where the stars are divided into four age bins. At low latitudes $\left(b \sim 3^{\circ}\right)$, the distance distribution generally consists of a single peak for all stellar ages, as expected since the arms of an X-shape only become sufficiently separated at higher latitudes. No split is present in the oldest stars even at higher latitudes, but one is visible for younger stars. The separation between the peaks increases and the depth of the minima between peaks increases with decreasing stellar age. Both the separation and the depth of the minima also become more prominent with increasing latitude. The prominence of the $\mathrm{X}$-shape depends strongly on stellar age, with the older stellar populations showing a weak (or no) bimodality and the younger populations exhibiting a strongly bimodal distance distribution.

We infer that the age dependence in the distribution of stars tracing the X-shape qualitatively explains the metallicity dependence of the split clump observed in the Milky Way, where the most metalrich stars show the largest bimodality and stars of $[\mathrm{Fe} / \mathrm{H}]<-0.5$ show only a single peak in their distance distribution. We identify the oldest stars in the simulation, which trace the X-shape weakly, if at all, with the metal-poor stars in the Galaxy. Conversely, we identify the more metal-rich stars in the Milky Way, which do trace the X-shape, with the slightly younger stars in the simulation.

The bottom row of Fig. 19 also shows that stars younger than 7 Gyr in the model also have a split red clump. The percentage of stars in each age bin at each latitude is indicated in each panel of Fig. 19. Stars younger than 7 Gyr contribute $<14$ percent of stars in this region, a number which reflects details of the model's evolution. In the Milky Way, this fraction would be even lower, since observations based on turnoff studies show that such stars contribute $<5$ percent of bulge stars (e.g. Ortolani et al. 1995; Kuijken \& Rich 2002; Zoccali et al. 2003; Sahu et al. 2006; Clarkson et al. 2008, 2011; Brown et al. 2010; Valenti et al. 2013). The crucial point is that an age difference of just $2 \mathrm{Gyr}$ is sufficient for 


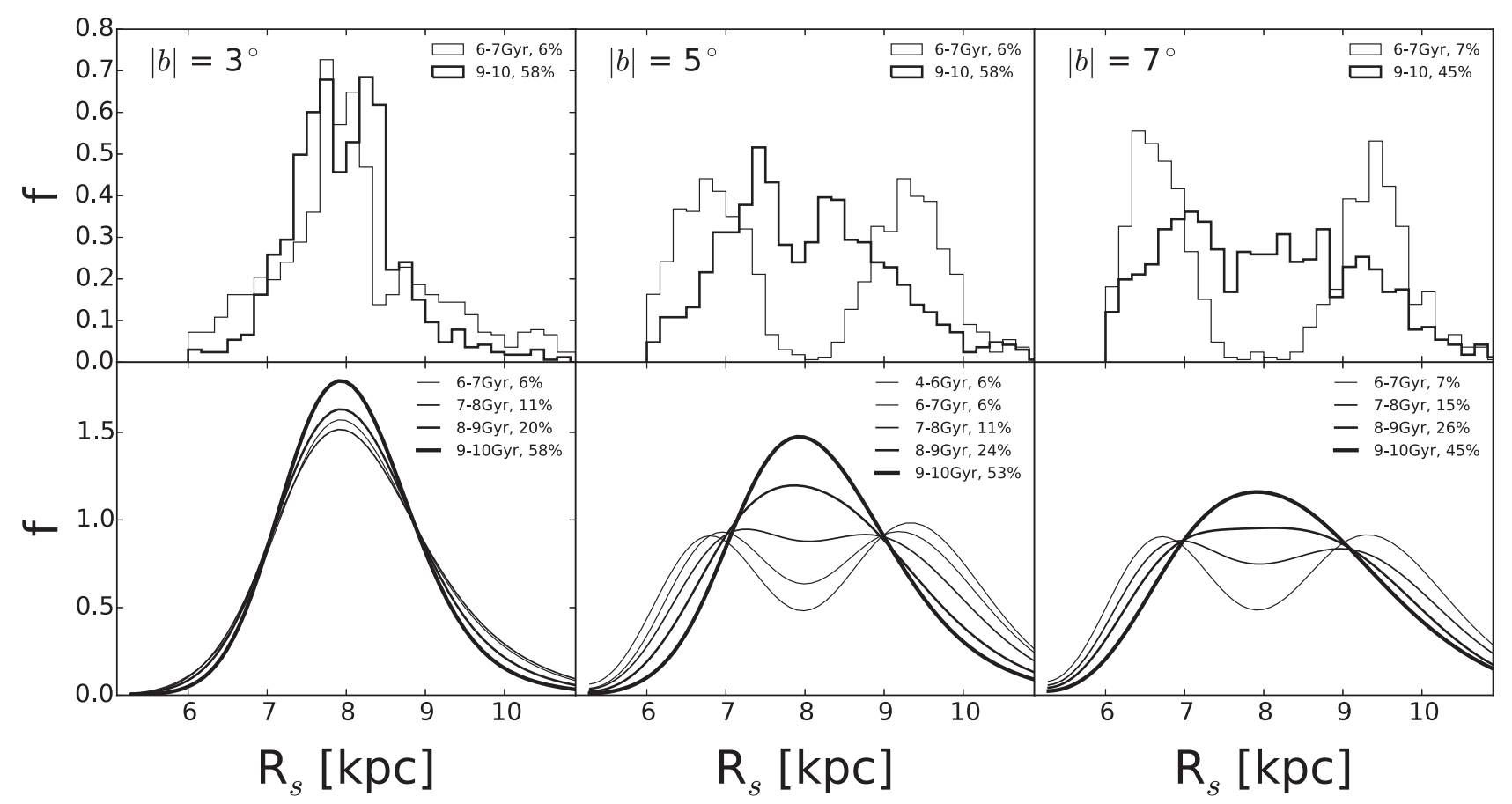

Figure 19. The distribution of red clump stars in the star-forming model, at distances from the Sun in the range $6 \leq R_{\mathrm{S}} / \mathrm{kpc} \leq 11$, at three different lines of sight on the minor axis, with the stars divided into age bins. Here, age is defined as $10 \mathrm{Gyr}-\tau$. Top: raw data with no observational error added. Bottom: the histograms convolved with the intrinsic scatter of the red clump magnitude of $\sigma=0.17$ dex. Younger (metal-rich) red clump stars would appear distributed on an X-shape, while older (metal-poor) ones would not, given the intrinsic scatter. More precise distance measurements however can reveal a weak bimodality also in metal-poor populations.

the bulge red clump distributions to change from a single peak to a bimodal distribution.

The absence of a bimodal distribution in stars older than $8 \mathrm{Gyr}$ is not absolute. We have convolved the magnitude distribution with an intrinsic scatter of $\sigma=0.17 \mathrm{mag}$. If the observational uncertainty were lower, then even the oldest population exhibits a weakly bimodal distribution. The top row of Fig. 19 presents examples of distance distributions unconvolved with any observational uncertainty. In this case, even the oldest population (age $>9 \mathrm{Gyr}$ ) has a bimodal distribution at $|b|=5^{\circ}$. A prediction that follows is that, with higher distance precision, a weaker split at lower metallicities may also be observed. A second prediction from the unsmoothed data is that, for the younger populations, the stars are distributed in two distinct distributions, corresponding to the arms of the X-shape, with a near zero density of stars in between. We emphasize that this particular prediction is for ages, not $[\mathrm{Fe} / \mathrm{H}]$ since at any age the distribution of $[\mathrm{Fe} / \mathrm{H}]$ is probably not perfectly single-valued.

\subsection{Interpretation of the different distributions of Milky Way bulge red clump stars and RR Lyrae}

The dependence of bar strength on age, shown in Figs 15 and 16, is directly relevant to interpreting the difference in the distribution of RR Lyrae and red clump stars noted by Dékány et al. (2013). The adoption of red clump stars as distance tracers includes the entire range of stellar ages that is found in the Milky Way bulge. On the other hand, RR Lyrae are old stars (Walker 1989). Dékány et al. (2013) investigated the spatial distribution of the oldest population of the bulge by measuring the distances to RR Lyrae based on their near-infrared light curves from VVV photometry. They found a distribution of RR Lyrae in the Galactic bulge that is quite round and exhibits little evidence of the bar morphology traced by the red clump stars. Fig. 20 shows the projected mean distance to red clump stars from Gonzalez et al. (2012) compared to that from RR Lyrae. We used the individual distances to the RR Lyrae from Dékány et al. (2013) to calculate a mean RR Lyrae distance using a Gaussian fit to the distance distribution in each line of sight. The projected distance is shown in Fig. 20 as well as the surface density contours using the individual distances of the entire RR Lyrae sample from Dékány et al. (2013). Dékány et al. (2013) interpreted the spheroidal distribution of this old population as evidence for a dynamically distinct population that formed separate from the disc in the bulge.

We assess the need for such a composite bulge scenario by measuring the mean distances to the simulated stars, in a similar way as done for the observations. We first select all the stars within the distance range $4 \leq \mathrm{R}_{\mathrm{s}} / \mathrm{kpc} \leq 12$ to sample the integrated population of the red clump stars observed across the extent of the bulge. We then consider only the oldest stellar population with $\tau \leq 0.5 \mathrm{Gyr}$ to obtain a sample representative of the oldest population of the bulge, corresponding to the population traced by the RR Lyrae. We then measure the mean distances to both samples in $(l, b)$-space at a fixed latitude of $3.5^{\circ} \leq b \leq 4.5^{\circ}$ in $1^{\circ}$-spaced longitude bins between $-9^{\circ} \leq l \leq+9^{\circ}$. As we did in comparing with the observed X-shape, in order to match the observational uncertainties, we first convert the line-of-sight distance of every star particle to an observed magnitude by adopting an absolute magnitude for the red clump of $M_{K}=-1.61 \mathrm{mag}$ (Alves 2000). The magnitude distribution of stars towards each line of sight is then convolved with a Gaussian with $\sigma=0.17 \mathrm{mag}$ in order to account for the intrinsic scatter of the bulge red clump population (Gonzalez et al. 2011; Gerhard \& Martinez-Valpuesta 2012). We then produce a Gaussian fit to the magnitude distribution towards each line of sight and retrieve the corresponding mean magnitude of the distribution. Finally, this mean magnitude value is converted back to a distance 


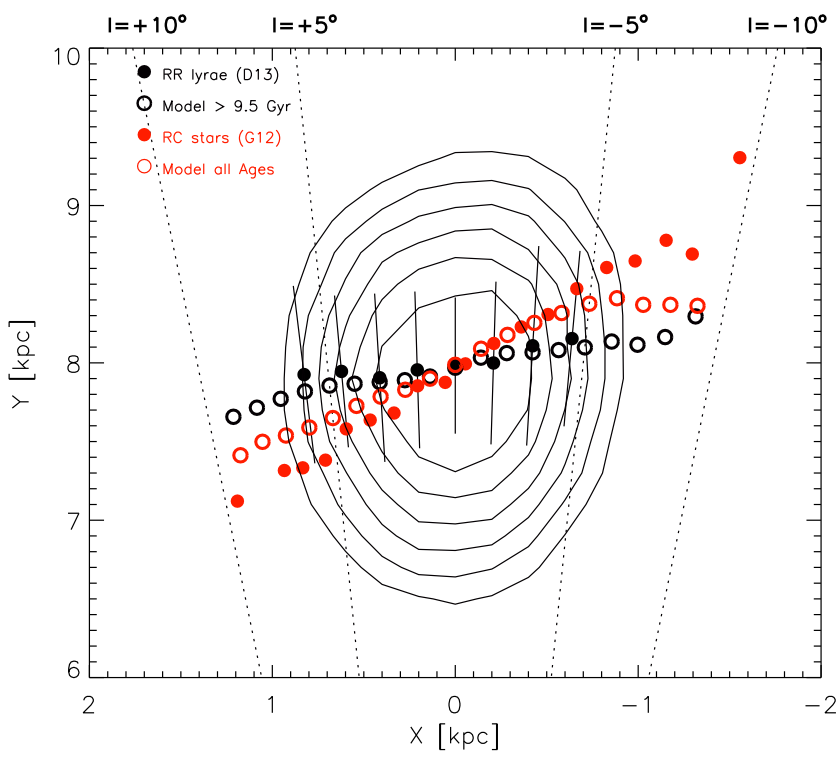

Figure 20. Face-on projection of the simulated mean line-of-sight distances for star particles at $3.5^{\circ}<b<4.5^{\circ}$ with ages $>9.5 \mathrm{Gyr}$ (black open circles) and for all the star particles (red open circles) in the star-forming simulation. The projected mean line-of-sight distances of RR Lyrae from Dékány et al. (2013) (black filled circles) for latitudes $3.5^{\circ} \leq b \leq 4.5^{\circ}$ and of red-clump stars in the same latitude range from Gonzalez et al. (2012, red filled circles) are also shown. The surface density contours of the full RR Lyrae sample of Dékány et al. (2013) are included in the plot. All distances, for simulated and observed stars, were calculated adopting an absolute magnitude of $M_{K}=-1.61$ mag in order to bring the model to the mean distance of the RR Lyrae. The simulation matches the weaker barred distribution of RR Lyrae and stronger bar in the red clump stars seen in the Milky Way's bulge.

using the same $M_{K}$ value. For the oldest stars, instead, we ignore the small distance uncertainties. Fig. 20 shows the mean positions projected on to the $(x, y)$-plane for both cases: including all the stars, and selecting only the oldest stars from the simulation.

Purely by measuring distances to the oldest stars, the distance profile becomes flatter than when including the entire range of ages, with a remarkable similarity to the RR Lyrae measurements in the Milky Way. Furthermore, when all the stars of the simulation are included, the distance profile becomes steeper and traces the position angle of the bar. Thus this result explains the different morphological signatures traced by red clump stars and RR Lyrae within the Galactic bulge, without the need for a second, dynamically distinct, component. Instead, the continuum of bar quadrupole moments, from weak to strong as a function of formation time, is able to explain the observed shape difference.

A natural interpretation of the nearly spheroidal distribution of the RR Lyrae stars in the Galactic bulge is that these are older than the bar itself. By the time the bar formed, they constituted a radially hotter stellar population, possibly as part of the stellar halo, and therefore did not acquire a strong quadrupole moment. A consequence of this interpretation is that the RR Lyrae should not exhibit a strong peanut shape.

Recently, Kunder et al. (2016) presented radial velocity measurements of 947 RR Lyrae from the Bulge RR Lyrae Radial Velocity Assay (BRAVA-RR) survey located in four bulge fields at Galactic latitude $-5^{\circ}<b<-3^{\circ}$ and longitude $|l|<4^{\circ}$. Kunder et al. (2016) concluded that the bulge RR Lyrae are part of a separate component, i.e. a classical bulge or inner halo, based on the null rotation observed in this population when compared to the RC stars. If this result is confirmed after extending the rotation curve of the RR Lyrae to larger Galactic longitudes $\left(|l|<10^{\circ}\right)$, it could suggest that the population traced by these stars is linked to the most metal-poor $([\mathrm{Fe} / \mathrm{H}]<-1.0)$ population of red clump stars that shows a similar kinematic behaviour as observed by the ARGOS survey (Ness et al. 2013b). Here, we showed that the oldest population found in the model follows a weakly barred distribution. However, we do not find a velocity difference between RR Lyrae and red clump stars as large as that seen in the BRAVA-RR data.

A more continuous change in the orientation of the bar with age has been measured in bulge Mira variables (Catchpole et al. 2016). Mira variables follow a period-age relationship, with short periods corresponding to older stars (Wyatt \& Cahn 1983), spanning a range of ages from $\sim 3 \mathrm{Gyr}$ to globular cluster ages. Catchpole et al. (2016) find that the younger ( $\lesssim 5 \mathrm{Gyr}$ ) Mira variables in the bulge at $(l, b)=\left( \pm 8^{\circ},-7^{\circ}\right)$ follow a clear bar structure, while the older ones are more spheroidal. The angle of the major axis of the distribution of Miras to the line of sight twists continuously with their period, as would be expected for populations with continuously varying quadrupole moments (e.g. Gerhard \& Martinez-Valpuesta 2012).

\subsection{Stellar kinematics}

We now compare the kinematics of the bulge as observed in ARGOS, selecting stars in the simulation to be within $3.5 \mathrm{kpc}$ of the Galactic Centre, as was done for red clump stars (with distance errors $<1.5 \mathrm{kpc}$ ) in ARGOS. The results of this section are not too sensitive to these distance cuts; removing the distance cuts changes the mean velocities and the dispersions by no more than 10 per cent.

The right column of Fig. 21 shows the rotation and dispersion profiles in the simulation compared to the ARGOS data, at latitudes of $b=5^{\circ}, 7.5^{\circ}$ and $10^{\circ}$. The rotation for both ARGOS and the simulation is cylindrical, with very little difference in rotation speed at different latitudes. Cylindrical rotation of the Galactic bulge has been observed by several studies (e.g. Howard et al. 2008; Kunder et al. 2012; Ness et al. 2013b, 2016b; Zoccali et al. 2014) and is a generic feature of $N$-body models of boxy bulges (Combes et al. 1990; Athanassoula \& Misiriotis 2002; Saha \& Gerhard 2013). The dispersion profiles of the observations and the simulation are also very similar, peaking in the centre and decreasing with increasing longitude. The peak velocity dispersion is observed near the centre $\left(b \sim 2^{\circ}\right)$ on the minor axis, as first reported by the GIBS survey (Zoccali et al. 2014), and decreases with latitude. The model's velocity dispersion at $b=2^{\circ}$ is $\sim 1.5$ times the velocity dispersion at $5^{\circ}$, which is comparable to the fractional difference reported in GIBS (Zoccali et al. 2014) and ARGOS (Ness et al. 2016a). Outside the boxy bulge, in the disc $\left(|l|>15^{\circ}\right)$, the dispersion is similar at all latitudes.

The ARGOS stars span a metallicity range of $-2<[\mathrm{Fe} / \mathrm{H}]<0.5$, although 95 per cent of stars have $[\mathrm{Fe} / \mathrm{H}]>-1$. The velocity dispersions of stars vary as a function of their metallicity (Babusiaux et al. 2010; Ness et al. 2013b, 2016b). We investigate these observed differences in the kinematics using the stellar age in the simulation as a proxy for $[\mathrm{Fe} / \mathrm{H}]$, as we did previously for the $\mathrm{X}$-shape analysis. The second column of Fig. 21 shows the kinematics of the metal-rich $([\mathrm{Fe} / \mathrm{H}]>-0.5)$ ARGOS stars, together with intermediate-age $(7 \mathrm{Gyr}<$ age $<8 \mathrm{Gyr}$ ) stars in the model. The model stars have kinematics that are qualitatively similar to the observational trends of the metal-rich stars, with a larger difference in velocity dispersion between $5^{\circ} \leq|b| \leq 10^{\circ}$, and a lower and flatter dispersion profile at higher latitudes, compared to all stars (left column). The $\sim 10$ per cent increase in the velocity dispersion 


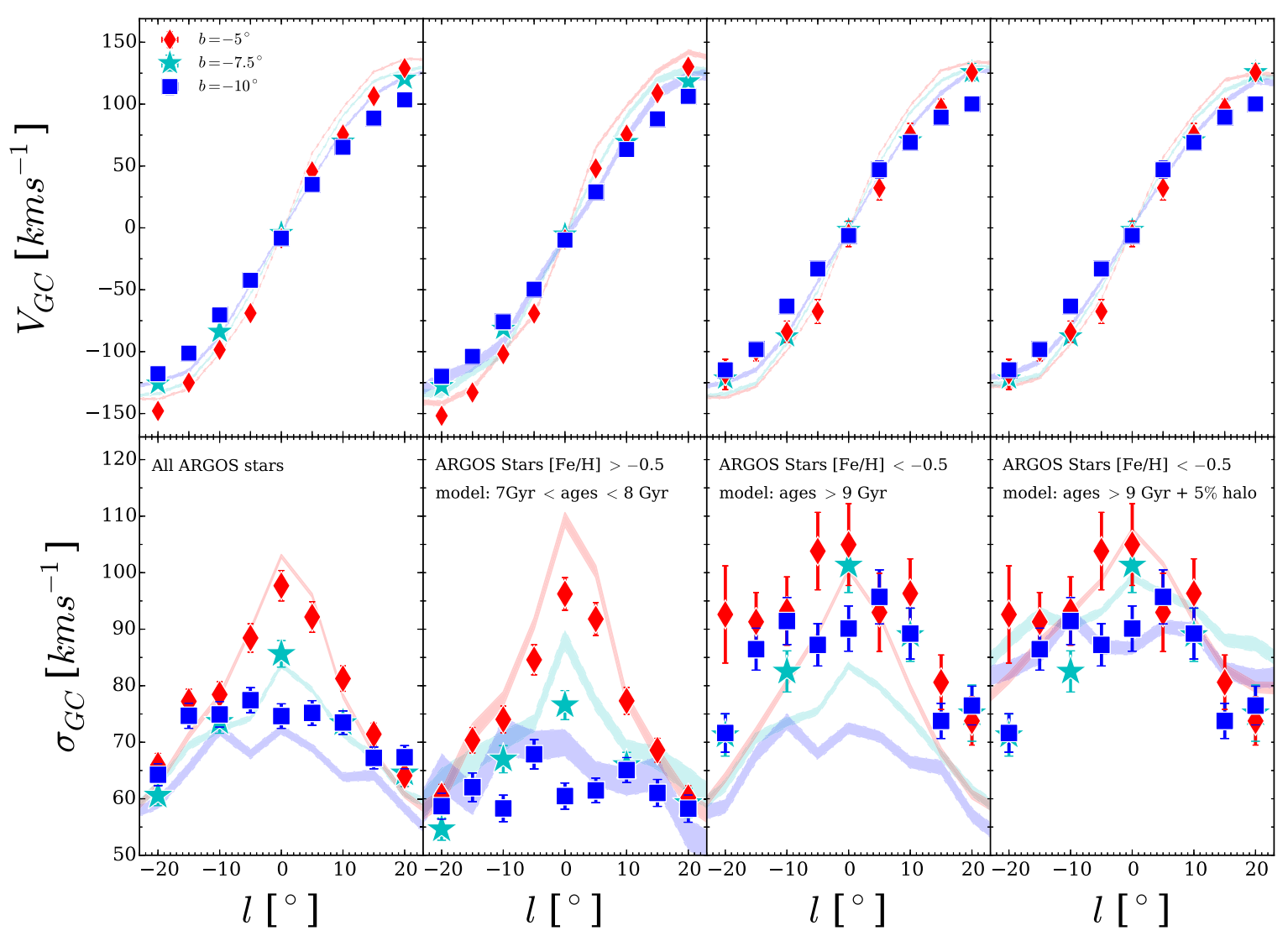

Figure 21. The ARGOS data (points with error bars) compared to the star-forming simulation (shaded region showing the sampling uncertainty; the shading uses the same colour scheme as the ARGOS data points to indicate latitude). Left: all stars in the simulation along a given line of sight. Second column: stars with $[\mathrm{Fe} / \mathrm{H}]>-0.5$ compared to model stars with ages between 7 and 9 Gyr. Third column: ARGOS stars with $[\mathrm{Fe} / \mathrm{H}]<-0.5$ compared with old model stars (age $>9 \mathrm{Gyr}$ ). Right: the same old stars in the model with a 15 per cent admixture of a hot population as described in the text. This fraction corresponds to 5 per cent of the stellar mass of the bulge; this small addition leads to an excellent match to the observed kinematics of the metal-poor stars.

at $b=-5^{\circ}$ of the intermediate-age stars relative to the full distribution is not observed in ARGOS for the more metal-rich stars. The origin of this discrepancy is unclear.

The third column of Fig. 21 compares the metal-poor $([\mathrm{Fe} / \mathrm{H}]<-0.5)$ ARGOS stars with old (age > 9 Gyr) stars in the model. The trends observed for this population, including the relatively high and flat velocity dispersion that changes slowly with latitude, are not reproduced by the model. We have explored a variety of age cuts in the model, and all of them fail to match these kinematic trends. This suggests that the model is missing a component that can produce these kinematics. A similarly hot population at low metallicity was found by Babusiaux (2016). In order to explore this population further, we note that ARGOS finds that stars with $[\mathrm{Fe} / \mathrm{H}]<-1$ have a $\sim 50$ per cent rotation velocity and an average velocity dispersion $\sim 120 \mathrm{~km} \mathrm{~s}^{-1}$ across $5^{\circ} \leq b \leq 10^{\circ}$ (component D of Ness et al. 2013a). We add such a population to the old stellar population in the model. The right column of Fig. 21 shows the outcome of adding 15 percent of stars with the properties of population D from ARGOS (i.e. rotation speed which is 50 per cent of the metal-rich stars and a dispersion of $120 \mathrm{~km} \mathrm{~s}^{-1}$ ) to the old population in the model. The qualitative match to the observations is now very good, with flat, nearly constant velocity dispersion with longitude and a relatively small drop with latitude. Bearing in mind that the $[\mathrm{Fe} / \mathrm{H}]<-0.5$ population accounts for 30 percent of all the ARGOS stars, and that the model needed only $\sim 15$ per cent of such stars to match this behaviour, we conclude that this component accounts for $\sim 5$ per cent of the mass of the central Milky Way. This additional population is most likely the stellar halo, which must also be present in the inner Milky Way. This low contribution of a hot component is significantly more stringent than the estimates of Shen et al. (2010) for the presence of a hot, unrotating component.

\subsection{Trends in the age distribution}

Fig. 22 shows the average age, $\langle$ age $\rangle$, (top panel) and the standard deviation in the age, $\sigma_{\text {age }}$, (middle panel) in $(l, b)$-space. The peak of $\langle$ age $\rangle$ is on the minor axis, although a small shift to positive $l$ due to the effect of perspective is evident. At the same location, $\sigma_{\text {age }}$ is at a minimum. The peak in $\langle$ age $\rangle$ and the minimum in $\sigma_{\text {age }}$ are due to the fact that it is the oldest stars that ascend the furthest on the bar's minor axis, giving rise to a vertical age gradient and a minimum in $\sigma_{\text {age }}$ as seen from the Sun. Younger populations are instead more peanut-shaped. The dominance of the oldest stars above the mid-plane is consistent with observations in the Milky Way (e.g. Ortolani et al. 1995; Kuijken \& Rich 2002; Zoccali et al. 2003; Sahu et al. 2006; Clarkson et al. 2008, 2011; Brown et al. 2010; Valenti et al. 2013). Ness et al. (2014) showed that, none the less, younger stars are also mixed in with the old stars, particularly when the most metal-rich populations are considered, even at latitudes $|b| \geq 2$. This qualitatively agrees with the finding of younger microlensed dwarf stars (Bensby et al. 2011, 2013). In the bottom panel of Fig. 22, we show the fraction, $f$, of all stars which have 


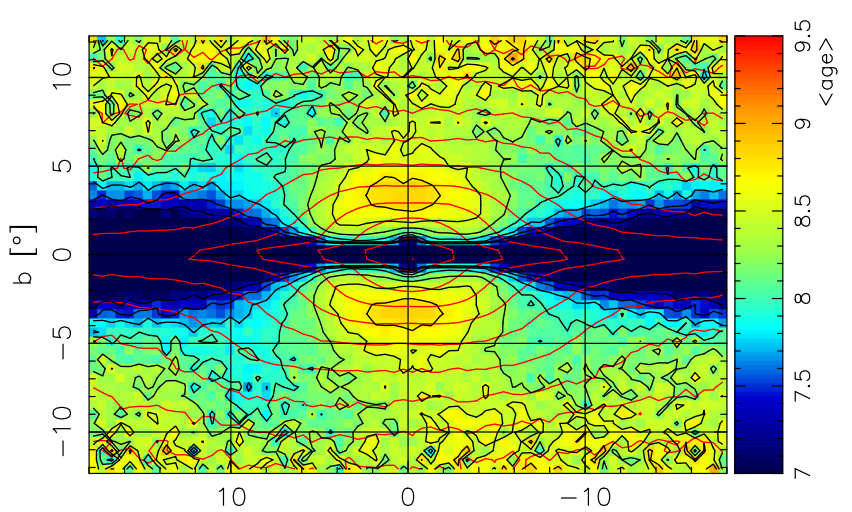

$\left[{ }^{\circ}\right]$

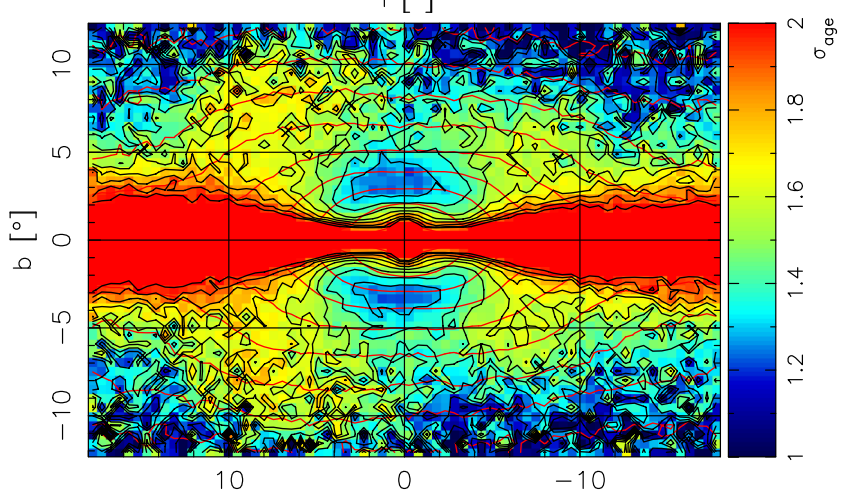

$1\left[^{\circ}\right]$

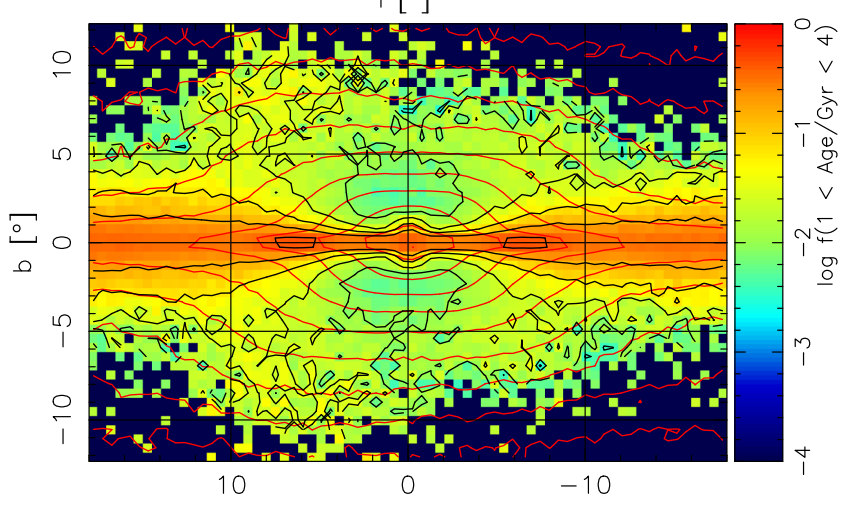

| [0]

Figure 22. Maps of $\langle$ age $\rangle$ (top), $\sigma_{\text {age }}$ (middle) and fraction of intermediateage stars (bottom) in $(l, b)$-space as seen from the Sun for the star-forming simulation. Only stars in the range $4 \leq R_{\mathrm{S}} / \mathrm{kpc} \leq 12$ are considered. Black contours show constant plotted quantity, while red contours show constant surface density. Note the difference between the age distribution and the density, and the dominance of old stars on the minor axis.

intermediate ages, which we define as those with ages between 1 and $4 \mathrm{Gyr}(9 \geq \tau / \mathrm{Gyr} \geq 6)$. Outside the disc, the intermediate-age population always accounts for less than 10 per cent of the stars, in good agreement with Milky Way estimates from isochrone fitting (e.g. Clarkson et al. 2011), but lower than estimated from microlensing (e.g. Bensby et al. 2013). Neglecting the disc, the youngest $\langle$ age $\rangle$ is at $l \simeq \pm 10^{\circ}$. As with 〈age $\rangle$ and $\sigma_{\text {age }}$, the minimum of $f$ is on the minor axis displaced slightly to positive $l$. There is a mismatch between the contours of surface density and those of $\langle$ age $\rangle, \sigma_{\text {age }}$ and $f$, with all these more pinched than the density.

Fig. 23 considers the difference in the intermediate-age distribution for near- and far-side stars. We define the near side as
$4 \leq R_{\mathrm{s}} / \mathrm{kpc} \leq 8$ and the far side as $8 \leq R_{\mathrm{s}} / \mathrm{kpc} \leq 12$. On the near side (left-hand panels of Fig. 23), the peak $\langle$ age $\rangle$ is at $l<0^{\circ}$, while more intermediate-age stars are at $l>0^{\circ}$ off the mid-plane. The situation is reversed on the far side. The interpretation of this result is that the $\mathrm{X}$-shape contains more intermediate-age stars $(\sim 6$ per cent in the model), and this structure exhibits a strong perspective effect. This distribution has an important consequence for microlensing. If microlensing events are dominated by sources on the far side of the Galaxy (Evans 1994; Zhao et al. 1996; Evans \& Belokurov 2002), then the fraction of lensed intermediate-age stars should be higher at $l<0^{\circ}$. We split the microlensed star sample of Bensby et al. (2013) at negative latitudes by age into three bins: young stars (age < $2 \mathrm{Gyr}$ ), old stars (age $>9 \mathrm{Gyr}$ ), with the remainder as intermediateage stars. Only three microlensed dwarfs are in the young bin, and we ignore them, assuming they are disc stars. There are 38 stars in the intermediate-age bin and 30 in the old bin. Remarkably, the intermediate-age stars comprise $\sim 50$ per cent of the total ( 24 of 49 stars) at $l>0^{\circ}$ and $\sim 74$ per cent of the total (14 of 19 stars) at $l<0^{\circ}$. Accounting for the uncertainties in the stellar ages, the fraction of intermediate-age lensed stars remains higher at negative longitudes compared with positive longitudes. Even if the sample of Bensby et al. (2013) is contaminated by foreground stars, as seems likely given the relatively high fraction of intermediate-age stars, the bulge remains the most likely source of an age asymmetry across longitude. While these results are suggestive, the sample remains too small to claim a significant detection of an asymmetry across longitude. Moreover, the microlensed stars for which spectra were obtained were selected heterogeneously (for example in the leftright asymmetry), making a detailed comparison with the model difficult.

\subsection{Trends in the chemical distribution}

Having considered the distributions of stars in the star-forming simulation as a function of age, we now turn to the distribution of stars by their metallicity, which is observationally more readily measureable. The absence of metallicity diffusion in this simulation leads to somewhat lower $\langle[\mathrm{Fe} / \mathrm{H}]\rangle$ and enhanced $\sigma_{[\mathrm{Fe} / \mathrm{H}]}$, which should be kept in mind when considering the results in this section, but the overall trends we focus on should remain valid.

The top-left panel of Fig. 24 presents a map of $\langle[\mathrm{Fe} / \mathrm{H}]\rangle$ in Galactic coordinates. Ignoring the nuclear disc at $|b|<1^{\circ}$, we find a metallicity gradient along the minor axis of $\sim-0.06 \pm 0.01 \mathrm{dex}$ deg. ${ }^{-1}$, in broad agreement with Gonzalez et al. (2011). A notable feature of this map is that it is more pinched in $\langle[\mathrm{Fe} / \mathrm{H}]\rangle$ than in the density. In the model, this difference is already apparent by $|b| \simeq 5^{\circ}$. In our model, it is the anticorrelation between in-plane dispersion and $[\mathrm{Fe} / \mathrm{H}]$ that sets up the long-lived vertical gradient. The top-right panel of Fig. 24 shows $\sigma_{[\mathrm{Fe} / \mathrm{H}]}$; this is also more pinched than the density distribution itself, although it does not have the boxy part at the centre that the $\langle[\mathrm{Fe} / \mathrm{H}]\rangle$ map does.

The middle row of Fig. 24 shows maps of $\langle[\mathrm{O} / \mathrm{Fe}]\rangle$ and $\sigma_{[\mathrm{O} / \mathrm{Fe}]}$ at 10 Gyr. Ignoring the nuclear disc $\left(|b|<1^{\circ}\right)$, a distinct $\alpha$-enhanced region is present at $|l|<5^{\circ}$ and $1^{\circ}<|b|<5^{\circ}$. As with $[\mathrm{Fe} / \mathrm{H}]$, the $\langle[\mathrm{O} / \mathrm{Fe}]\rangle$ and $\sigma_{[\mathrm{O} / \mathrm{Fe}]}$ maps are more pinched than is the density map.

Fig. 23 showed that selecting stars on the near or far side produces differences in the mean age distribution across $l=0^{\circ}$. Since age anticorrelates with $[\mathrm{Fe} / \mathrm{H}]$, metallicity maps of near versus far sides should also be asymmetric across $l=0^{\circ}$. The bottom row of Fig. 24 shows such maps; as expected there is an asymmetry across the minor axis, with intermediate-metallicity stars extending to larger 

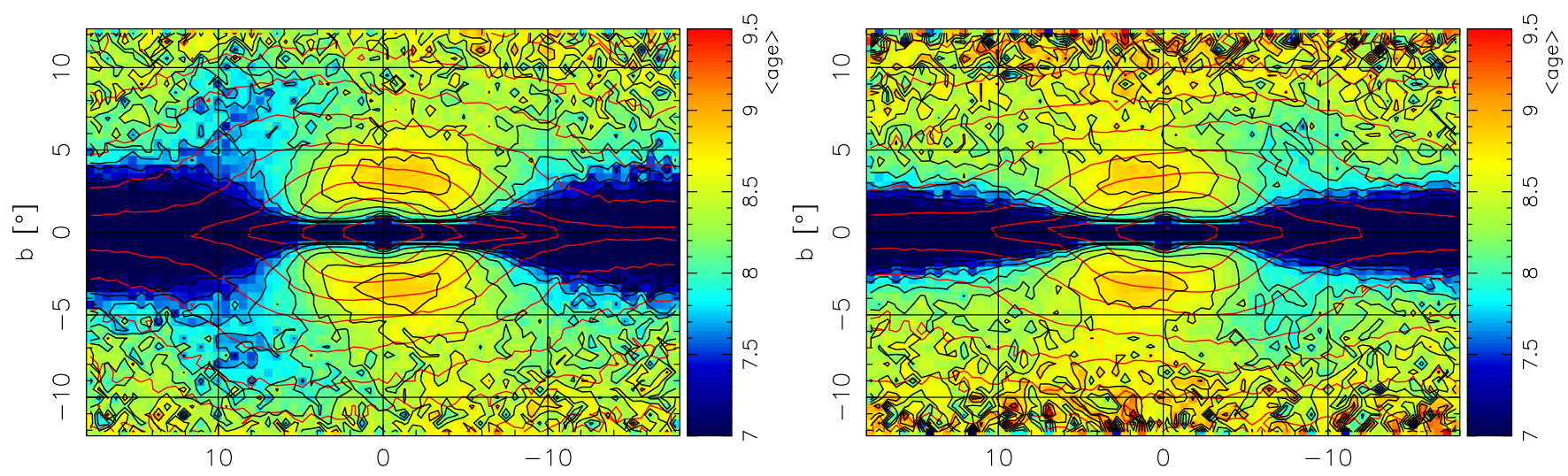

$1\left[^{\circ}\right]$
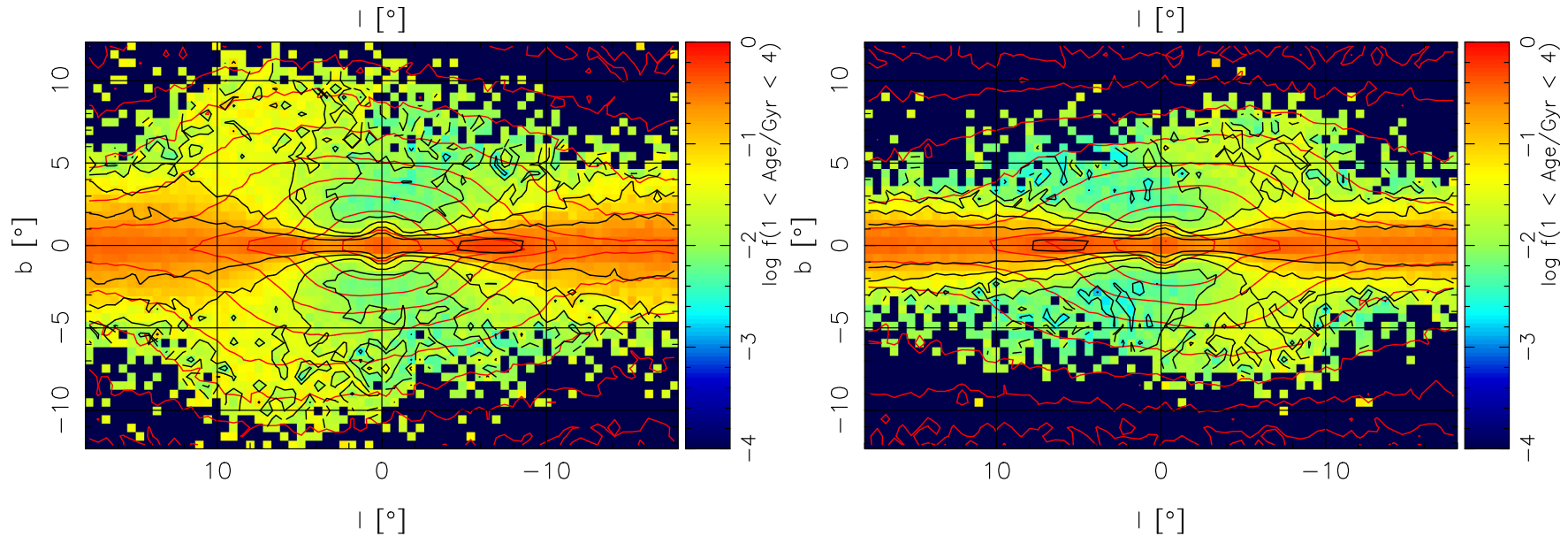

$\left[{ }^{\circ}\right]$

Figure 23. Near- versus far-side stellar age distributions in the star-forming simulation. Top row: maps of $\langle$ age $\rangle$. Bottom row: maps of fraction of intermediateage $(1 \mathrm{Gyr} \leq$ age $\leq 4 \mathrm{Gyr})$ stars. The maps on the left are for near-side stars $\left(4 \leq R_{\mathrm{s}} / \mathrm{kpc} \leq 8\right)$, while those on the right are for the far-side stars $\left(8 \leq R_{\mathrm{S}} / \mathrm{kpc}\right.$ $\leq 12$ ). Black contours show constant plotted quantity while red contours show constant surface density of the population under consideration. Away from the minor axis younger stars contribute most on the side where the arms of the X-shape are located. Thus, the greater vertical extent of the intermediate-age stars is at $l>0^{\circ}$, a perspective effect.

latitude at $l>0^{\circ}$ on the near side and at $l<0^{\circ}$ on the far side. However, this difference is not as prominent as in the ages. As with ages, this asymmetry will manifest in microlensed stars. Using the microlensing data of Bensby et al. (2013), we find that there is a higher average metallicity for lensed stars at $l<0^{\circ}$ than at $l>0^{\circ}$, but again the number of stars is too small for this result to be very significant.

Ness et al. (2013a) showed that, in the ARGOS survey, the fraction of metal-rich (their component A) stars decreases with latitude. In contrast, the fraction of metal-poor stars (their component $\mathrm{C}$ ) increases relative to the intermediate-metallicity stars although they remain less abundant. Fig. 25 shows maps of the fraction of stars in the same three broad metallicity bins A, B and C as in Ness et al. (2013a, we stress that these are only convenient bins, not distinct structural components). The metal-rich population $\mathrm{A}$ is dominant at low latitudes, but drops off rapidly with increasingly latitude, as found also by Ness et al. (2013a). The metal-poor population C instead follows the opposite trend, representing a smaller population at low latitudes, but increases with latitude. The intermediatemetallicity population B dominates everywhere except near the disc where population A dominates. These are all trends that are in agreement with the findings of Ness et al. (2013a).

Recently, Zoccali et al. (2017) parametrized the metallicity distribution in each of the 26 fields of the GIBS survey into a metal-poor and a metal-rich components. They presented a density map for each of the two components for $-10^{\circ}<l<10^{\circ}$ and $-10^{\circ}<b<10^{\circ}$ showing that the metal-poor population is more centrally concentrated than the metal-rich one, and has a more axisymmetric spatial distribution. On the other hand, the metal-rich population has a box-like distribution. These trends are consistent with the results of Fig. 25.

\section{DISCUSSION}

The separation by the bar of different stellar populations on the basis of their in-plane kinematics presents a novel explanation for the origin of many of the trends seen in the Milky Way's bulge, which have seemed to require more than one formation mechanism. Because this mechanism has never been described before, we coin the term 'kinematic fractionation' to refer to it.

Kinematic fractionation circumvents the need for two or more components to explain the trends observed in the bulge by employing a continuum of stellar populations with different in-plane random motions, a very natural outcome of internal evolution. Heating can be accomplished in a variety of ways, including star-forming clumps, spirals, lopsidedness and other perturbations, supernova feedback, gas turbulence and even interactions, but there is no need to generate a separate population (a thick disc or a classical 

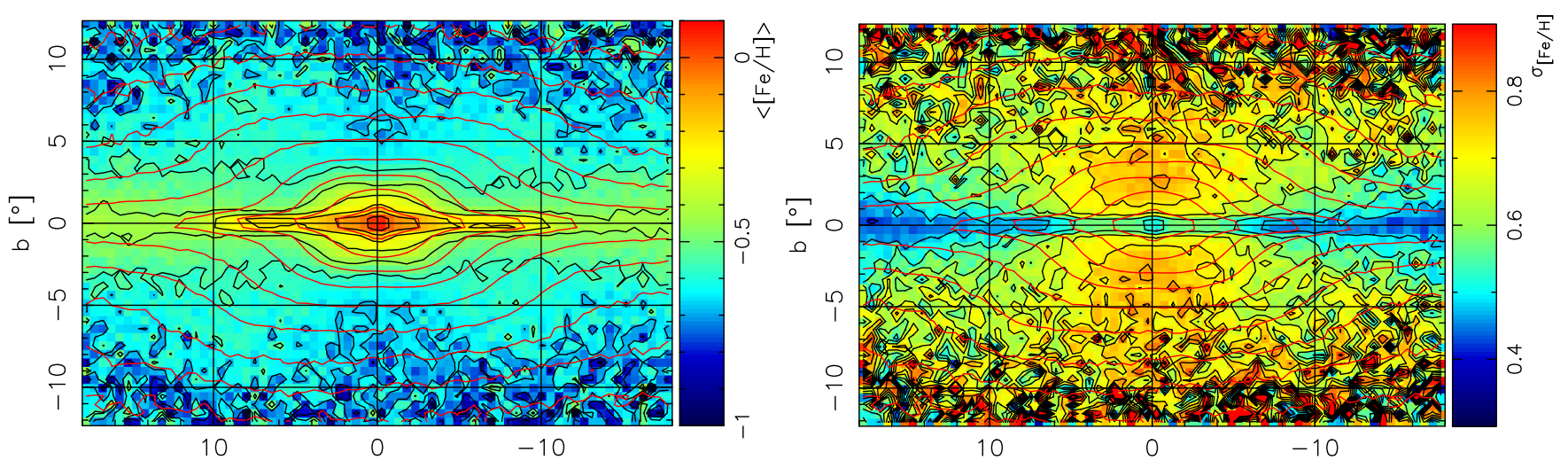

$1\left[^{\circ}\right]$
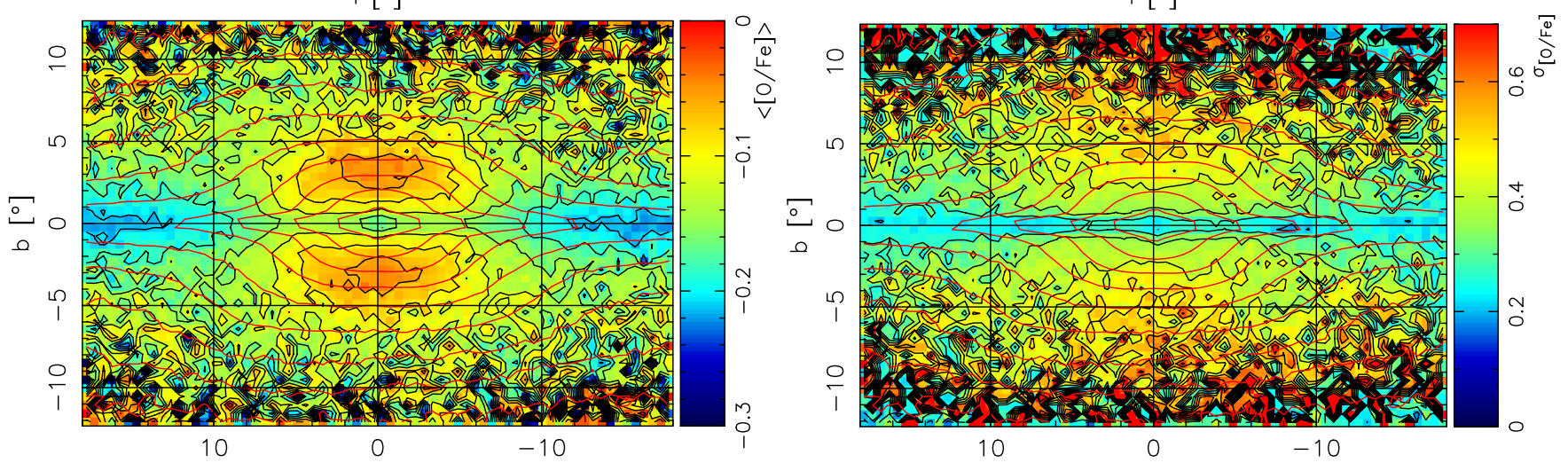

$1\left[^{\circ}\right]$
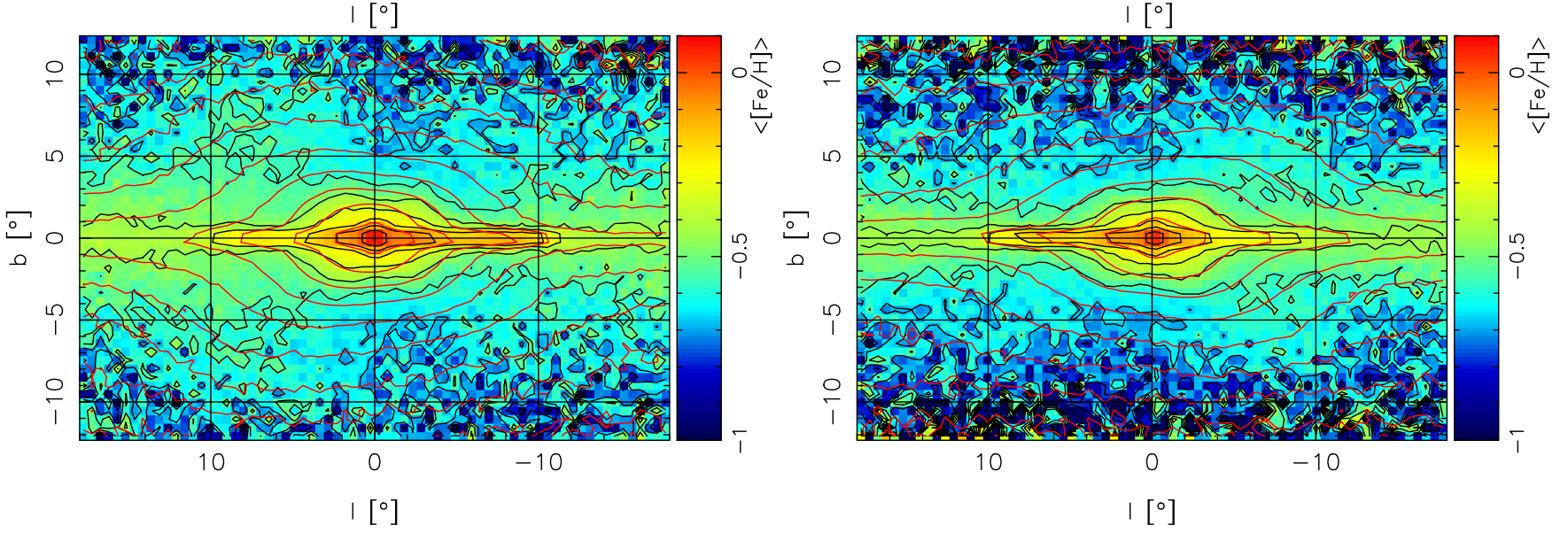

Figure 24. The stellar chemistry in the star-forming simulation. Top: maps of $\langle[\mathrm{Fe} / \mathrm{H}]\rangle$ (left) and $\sigma_{[\mathrm{Fe} / \mathrm{H}]}$ (right) in $(l, b)$-space as seen from the Sun. Only stars in the range $4 \leq R_{\mathrm{S}} / \mathrm{kpc} \leq 12$ are considered. Middle: same as top row for [O/Fe]. Bottom: maps of $\langle[\mathrm{Fe} / \mathrm{H}]\rangle$ for near-side (left) and far-side (right) stars. Black contours show constant plotted quantity, while red contours show constant surface density of the population under consideration. Note the vertical gradients in $\langle[\mathrm{Fe} / \mathrm{H}]\rangle$ and $\langle[\mathrm{O} / \mathrm{Fe}]\rangle$ and the mismatch between density and chemistry contours.

accreted bulge) in the process. Moreover, early discs may form hotter directly (e.g. Bird et al. 2013). As we showed in the star-forming simulation, at early times even $2 \mathrm{Gyr}$ is enough time to produce a significant difference in the random motions between younger and older stars, and in the Milky Way the bar may have required even longer to form. Nor do the trends observed in the Milky Way require that different spatial regions of the disc contribute to different parts of the bulge. For instance, one of the key differences between our model and earlier work is that the vertical metallicity gradient results not from a radial gradient in the initial system, but from the correlation between age (and metallicity) and kinematics at bar formation. This results in a long-lived vertical gradient.

The star-forming simulation we have compared the Milky Way to is not a particularly good match to it. Thus, the results presented here have focused on trends, not on detailed matches to observations. On the other hand, the very fact that this quite generic model produces trends that are also seen in the Milky Way argues very forcefully that the model captures the essential physics that produces these non-trivial trends, unless we are prepared to accept a quite remarkable coincidence. 


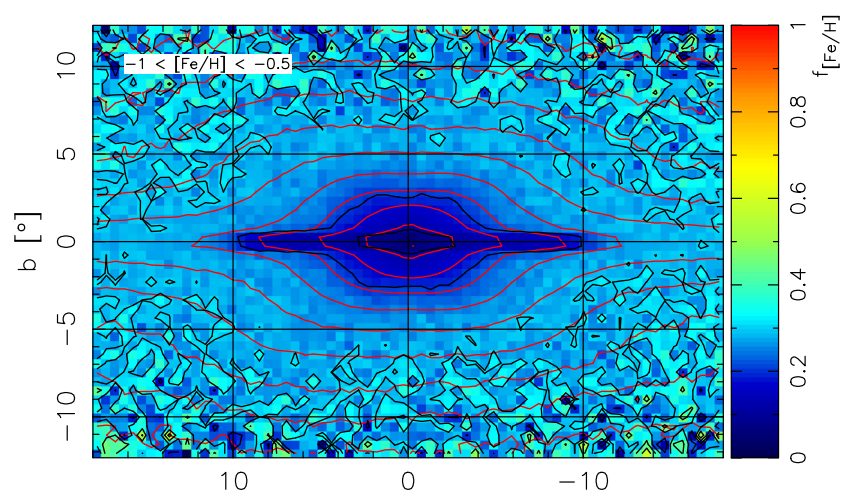

I [0]

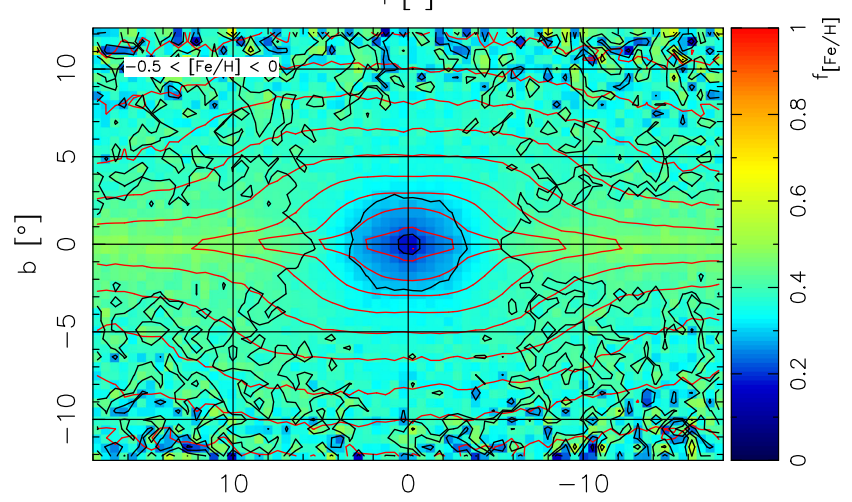

$\left[{ }^{\circ}\right]$

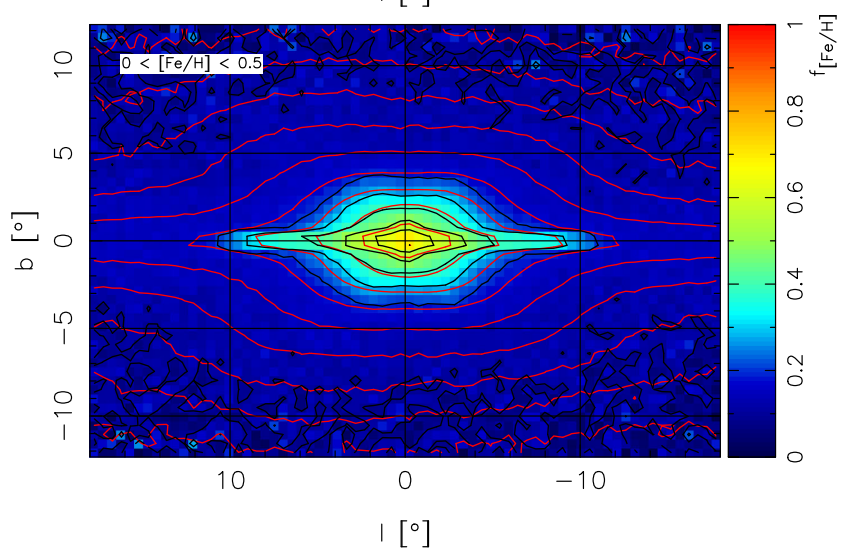

Figure 25. The fraction of stars in the star-forming simulation in different metallicity bins (as indicated at top left) corresponding to the populations A (top), B (middle) and C (bottom) of Ness et al. (2013a). Black contours show constant fractions, while red contours show constant overall surface density. Metal-rich stars dominate at small $|b|$, while the intermediate-metallicity stars dominate everywhere else, as in ARGOS.

\subsection{Is a thick disc necessary?}

Implicit in our interpretation of the bulge of the Milky Way is that there is a continuum of properties between old stars and those slightly younger, most importantly in their in-plane kinematics. Inevitably, a population that has hotter radial kinematics also has hotter vertical kinematics, as is evident for the star-forming simulation in Fig. 14. Simulations (e.g. Brook et al. 2012; Bird et al. 2013; Stinson et al. 2013; Grand et al. 2016) have suggested that discs form 'upside-down', i.e. thick initially slowly settling into thinner structures. This view is consistent with the observed large gas velocity dispersions due to disordered motions in high-redshift galaxies (e.g. Law et al. 2009; Gnerucci et al. 2011; Kassin et al. 2012; Wisnioski et al. 2015), while the lack of flaring in the thick disc of the Milky Way (Bovy et al. 2016) is also suggestive of such formation. Thus, it may be that a thick disc was present already by the time of bar formation. An ab initio thick disc will still experience vertical heating by the bar and the extent of this will be determined by the in-plane motion. Moreover, the in-plane motion of such a thick disc will determine whether its stars form part of the X-shape (see Section 3.2). A pre-existing thick disc will therefore produce the trends observed in the Milky Way's bulge (Bekki \& Tsujimoto 2011; Di Matteo et al. 2015), via kinematic fractionation.

However, the oldest population in this simulation does not constitute an especially thick disc at $2 \mathrm{Gyr}$, before the bar has formed. Therefore, we argue that a pre-existing thick disc at the time of bar formation, whether as part of a continuum with the thin disc (Brook et al. 2004; Schönrich \& Binney 2009; Loebman et al. 2011; Bovy, Rix \& Hogg 2012; Bird et al. 2013), or as the separate distinct structure envisaged by Bekki \& Tsujimoto (2011), is not necessary to produce the chemical trends observed in the Milky Way bulge. However, it is not inconceivable that the oldest population evolves into what, structurally, is consistent with the present-day thick disc. Indeed in the star-forming simulation, the oldest population evolves to a scaleheight of $1 \mathrm{kpc}$ at a radius of $5 \mathrm{kpc}$. Thus, while the observed bulge trends are consistent with a formation with a thick disc already present (e.g. Bekki \& Tsujimoto 2011), a thick disc is not necessary to explain them.

\subsection{Consequences for external galaxies}

The evolution we have outlined is very general and should occur in external galaxies that have evolved in isolation. Additional support for kinematic fractionation having played a dominant role in shaping bulges can be obtained by testing for similar trends in external galaxies. A straightforward test comes from the metallicity distribution compared with the density distribution in edge-on galaxies. As with the Milky Way, a map of mass-weighted $\langle[\mathrm{Fe} / \mathrm{H}]\rangle$ will appear more pinched-/peanut-shaped than the density distribution off the mid-plane. Fig. 26 illustrates this behaviour and shows that the difference remains detectable to a bar angle of $60^{\circ}$ to the line of sight, which is also roughly the limit at which a peanut shape can be detected.

Fig. 27 presents an age map for the model. This exhibits a more prominent peanut-shape than in the density distribution. However, the dynamic range outside the disc is relatively small, $\sim 2 \mathrm{Gyr}$, so may be difficult to measure directly.

\subsection{Future prospects}

A number of future spectroscopic and photometric surveys will offer critical comparisons to test our model in more detail. These include APOGEE-2 (Majewski et al. 2016), 4MOST (de Jong et al. 2014), MOONs (Cirasuolo et al. 2014) and LSST (Ivezic et al. 2008). APOGEE-2 (2016-2020), observes at high resolution in the near-IR so that it can efficiently observe the bulge at low latitudes, past the obscuring dust which limits optical observations. MOONS (2019) will later offer the possibility to map the inner Galaxy at a similar resolution and wavelength but it will make use of the $8 \mathrm{~m}$ aperture of the Very Large Telescope (VLT) to reach deeper, to study fainter stars. The highly complementary parameter space mapped by these surveys, measuring radial velocities and abundances of more than 15 elements for thousands of bulge stars, will be supplemented by the astrometric information from the near-IR photometric mapping 

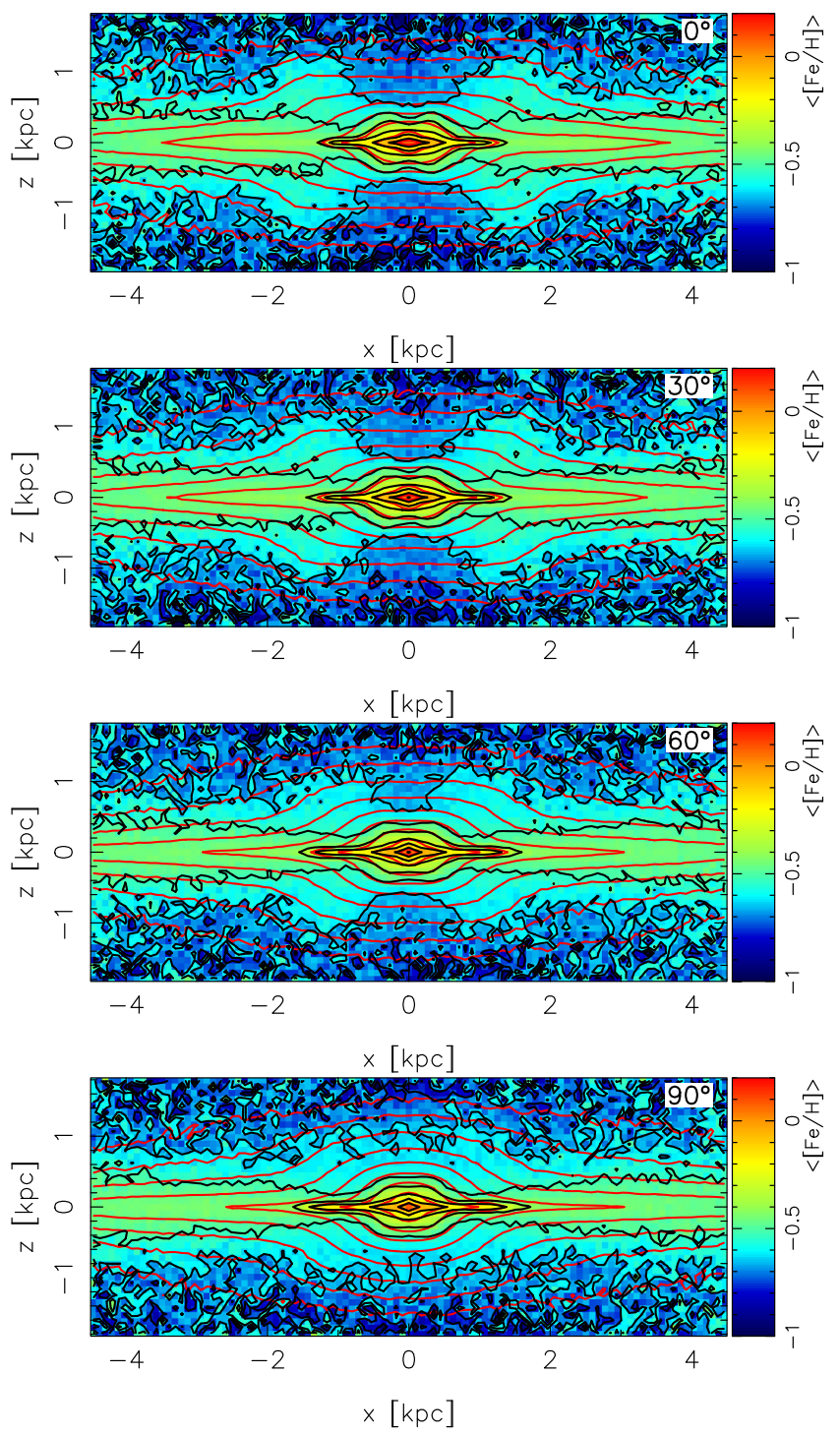

Figure 26. Maps of $\langle[\mathrm{Fe} / \mathrm{H}]\rangle$ (black contours and colours) and surface density (red contours) in the star-forming simulation seen edge-on with the bar side-on (top), end-on (bottom) and two intermediate orientations (middle two panels). The bar's orientation is indicated in the top right of each panel. These maps predict the trends in the metallicity distribution of external B/P-bulge galaxies.

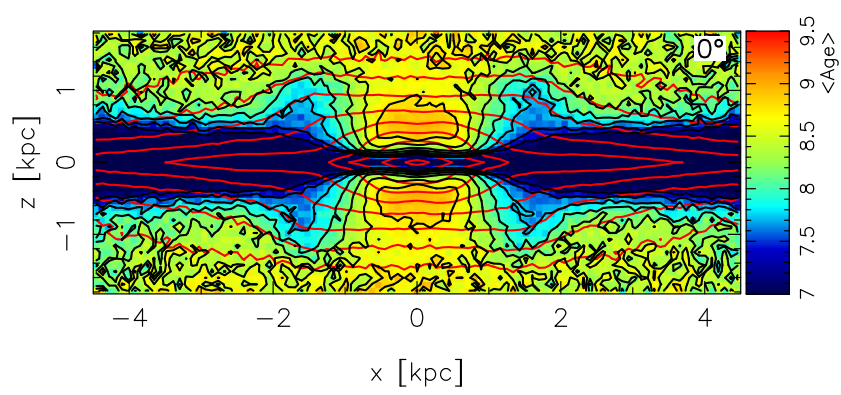

Figure 27. Map of $\langle$ age $\rangle$ (black contours and colours) and surface density (red contours) in the star-forming simulation seen edge-on with the bar side-on. of the VVV survey. The kinematics, chemical abundances, proper motions and even spectroscopic age measurements of large number of stars (Martig et al. 2015; Ness et al. 2016a), in addition to those of other Galactic components provided by optical surveys such as Gaia, 4MOST, WEAVE and LSST, will provide the critical constraints on our model, helping us understand the detailed formation history of the Galaxy. In this context, our model also presents the possibility of defining optimal strategies during the planning stages of forthcoming surveys.

Our predictions are general and not specific to the Milky Way and so comparisons to boxy bulges in other spiral galaxies are also very important. Modern Integral Field Unit spectrographs and their dedicated surveys offer a great opportunity for understanding the bulge of the Milky Way in context (Gonzalez \& Gadotti 2016), by measuring the morphological aspects of other spiral galaxies and prevalence of mergers compared to secular evolution at high redshift (e.g. Gadotti et al. 2015)

Our results also point the way forward in terms of future efforts to simulate the Milky Way in detail. Since simulations with full gas physics are subject to much more stochasticity, and are computationally expensive, pure $N$-body simulations will continue to play an important role in trying to understand the bulge of the Milky Way. Past pure $N$-body simulations of the Milky Way (e.g. Fux 1997; Shen et al. 2010; Martinez-Valpuesta \& Gerhard 2011) have produced good matches to the density distribution and kinematics of the bulge. Studying the age and metallicity distributions of such models requires assigning to particles a population tag (age/chemistry) in some way. Martinez-Valpuesta \& Gerhard (2013) used such a technique to match the observed vertical $[\mathrm{Fe} / \mathrm{H}]$ gradient; in their approach the metallicity of a particle was a function of its initial orbital radius. We propose that a more useful approach to assigning a population tag to particles is based on their initial orbital circularities, rather than on their initial position.

\subsection{Summary}

Our main conclusions can be summarized as follows

(i) Bars can efficiently separate disc stellar populations that differ by their initial in-plane kinematics (see Section 3). Hot populations form bars with weak quadrupoles, become vertically thick, particularly at the centre, and are box-shaped, while the cooler populations host stronger bars, are thinner at the centre and thus produce a peanut shape. We refer to this separation of populations as kinematic fractionation.

(ii) The early rapid evolution of discs naturally imprints correlations (or anticorrelations) between age, $\sigma_{R},[\mathrm{Fe} / \mathrm{H}]$ and $[\mathrm{O} / \mathrm{Fe}]$, which leads to relations between $\sigma_{R}$ and chemistry (see Section 4.1). After bar formation, kinematic fractionation leads to different populations having different bar strengths, vertical distributions and B/P-bulge strengths, naturally without the need for a separate origin for different populations. Instead the properties of the bulge vary continuously with age. The underlying reason for these differences is primarily the difference in the initial $\sigma_{R}$ and its strong dependence on age in young galaxies (see Section 4.2).

(iii) Kinematic fractionation also leads to the $\mathrm{X}$-shape that is stronger in relatively younger (metal-rich) stars than in the older (metal-poor) ones. In our star-forming model, a difference of just 2 Gyr in age is sufficient to alter the final distribution of stars from a single peak to a bimodal distribution. This is also consistent with observations in the Milky Way (Ness et al. 2012; Uttenthaler et al. 2012; Rojas-Arriagada et al. 2014). Moreover we predict that, 
for tracers with small distance uncertainties, old populations will still appear bimodal, while younger populations will be well separated (see Section 5.2).

(iv) Kinematic fractionation of a hot, older population and a cooler, younger population produces a weaker old bar population and a stronger young bar population, even if the difference in age is only $\sim 2$ Gyr. This is consistent with what is observed in the Milky Way, where the RR Lyrae trace a weak bar, while red clump stars trace a stronger bar (Dékány et al. 2013). In addition, bulge Mira variables show a smooth variation from strongly barred to more spheroidal distribution as a function of their age (Catchpole et al. 2016, see Section 5.3).

(v) Comparing with the ARGOS data (Ness et al. 2013b), the model is able to match the overall kinematics and those of the metal-rich population. Matching to the kinematics of the metalpoor population, with its relatively flat velocity dispersion which varies slowly with latitude, requires the addition of 15 percent of stars in a hot, slowly rotating component. This slowly rotating population amounts to $\sim 5$ per cent of all bulge stars. Assuming a bulge-to-total stellar mass ratio of $\sim 25$ per cent (Bland-Hawthorn $\&$ Gerhard 2016), this amounts to 1.25 per cent of the stellar mass of the Milky Way. We attribute these stars to a metal-poor halo, leaving no room for a classical bulge. This conclusion is in agreement with the independent result derived by Kormendy et al. (in progress and private communication) that the Milky Way has no classical bulge component: aside from the boxy bulge, near the centre they find only a nuclear star cluster and a small discy pseudobulge. Bland-Hawthorn \& Gerhard (2016) review some of the trends which have been used to argue for a bulge component built through mergers (a 'classical bulge' component); we have shown here that these trends can be explained without such an accreted bulge (see Section 5.4).

(vi) A vertically declining $[\mathrm{Fe} / \mathrm{H}]$ gradient, and vertically rising $[\mathrm{O} / \mathrm{Fe}]$ gradient, are established largely as a result of the different vertical extents of different ages. A declining $[\mathrm{Fe} / \mathrm{H}]$ gradient has been found in the Milky Way (e.g. Zoccali et al. 2008; Gonzalez et al. 2011; Johnson et al. 2013). Separating populations into broad metallicity bins, as used in ARGOS (Ness et al. 2013a), we showed that at low latitudes the metal-rich population dominates with the low-metallicity and intermediate-metallicity bins providing a smaller contribution. The contribution of the metal-rich population drops rapidly at larger latitudes, with that of the low- and intermediate-metallicity bins rising. The intermediate-metallicity bin dominates at large latitudes. These trends are in agreement with observations in the Milky Way (Ness et al. 2013a, see Section 5.6).

(vii) Because the minor axis is dominated by old, metal-poor stars, a prediction of the kinematic fractionation model is that the shape of the $\langle[\mathrm{Fe} / \mathrm{H}]\rangle$ (or $\langle[\mathrm{O} / \mathrm{Fe}]\rangle$ or $\langle$ age $\rangle$ ) map in the $(l, b)$-plane will appear more pinched/peanut-shaped than the density distribution. These trends will need to be verified in future surveys (see Sections 5.5 and 5.6).

(viii) We predict that microlensed dwarf stars on the far $\left(R_{\mathrm{S}}>8 \mathrm{kpc}\right)$ side of the Galaxy will contain more intermediateage, metal-rich stars at negative longitudes compared with positive longitudes. The data of Bensby et al. (2013) suggest such trends, but the number of spectroscopically measured microlensed dwarfs is still too small to confirm this result (see Sections 5.5 and 5.6).

(ix) A simple test of the kinematic fractionation model is provided by external edge-on barred galaxies with $\mathrm{B} / \mathrm{P}$ bulges, for which maps of $\langle[\mathrm{Fe} / \mathrm{H}]\rangle$ will be more strongly pinched/peanutshaped than the density distribution itself (see Section 6.2).

\section{ACKNOWLEDGEMENTS}

VPD is supported by STFC Consolidated grant no. ST/M000877/1. $\mathrm{MN}$ is funded by the European Research Council under the European Union's Seventh Framework Programme (FP 7) ERC Grant Agreement no. 321035. MZ and DM acknowledge support by the Ministry of Economy, Development, and Tourism's Millennium Science Initiative through grant IC120009, awarded to The Millennium Institute of Astrophysics (MAS), by the BASAL-CATA Center for Astrophysics and Associated Technologies PFB-06, Fondecyt Regular Proyects 1130196 and 1150345 and (MZ) by CONICYT's PCI grant DPI20140066. We acknowledge support from the ESF Exchange Grant (number 4650) within the framework of the ESF Activity entitled 'Gaia Research for European Astronomy Training'. The star-forming simulation used in this paper was run at the High Performance Computing Facility of the University of Central Lancashire. The pure $N$-body simulations were run at the DiRAC Shared Memory Processing system at the University of Cambridge, operated by the COSMOS Project at the Department of Applied Mathematics and Theoretical Physics on behalf of the STFC DiRAC HPC Facility (www.dirac.ac.uk). This equipment was funded by BIS National E-infrastructure capital grant ST/J005673/1, STFC capital grant ST/H008586/1 and STFC DiRAC Operations grant ST/K00333X/1. DiRAC is part of the National E-Infrastructure. We are deeply grateful to Larry Widrow for his support with using GALACTICS, including providing us with a development version that allows for two discs of different thickness. VPD acknowledges with great pleasure the support of the Pauli Center for Theoretical Studies, which is supported by the Swiss National Science Foundation (SNF), the University of Zürich and ETH Zürich. He particularly thanks George Lake for arranging for his sabbatical visit during which time this paper was completed. We thank Sandro Tacchella, Reynier Peletier, Peter Erwin and Marc Balcells for useful discussions. We thank the anonymous referee for useful comments that have helped improve the paper.

\section{REFERENCES}

Alves D. R., 2000, ApJ, 539, 732

Athanassoula E., 2002, ApJ, 569, L83

Athanassoula E., 2005, MNRAS, 358, 1477

Athanassoula E., Misiriotis A., 2002, MNRAS, 330, 35

Aumer M., Binney J. J., 2009, MNRAS, 397, 1286

Aumer M., Schönrich R., 2015, MNRAS, 454, 3166

Babusiaux C., 2016, PASA, 33, e026

Babusiaux C. et al., 2010, A\&A, 519, A77

Behroozi P. S., Wechsler R. H., Conroy C., 2013, ApJ, 770, 57

Bekki K., Tsujimoto T., 2011, MNRAS, 416, L60

Bensby T. et al., 2011, A\&A, 533, A134

Bensby T. et al., 2013, A\&A, 549, A147

Binney J., Tremaine S., 1987, Galactic Dynamics. Princeton Univ. Press, Princeton, NJ

Binney J., Gerhard O. E., Stark A. A., Bally J., Uchida K. I., 1991, MNRAS, 252,210

Binney J., Gerhard O., Spergel D., 1997, MNRAS, 288, 365

Bird J. C., Kazantzidis S., Weinberg D. H., Guedes J., Callegari S., Mayer L., Madau P., 2013, ApJ, 773, 43

Bland-Hawthorn J., Gerhard O., 2016, ARA\&A, 54, 529

Bovy J., Rix H.-W., Hogg D. W., 2012, ApJ, 751, 131

Bovy J., Rix H.-W., Schlafly E. F., Nidever D. L., Holtzman J. A., Shetrone M., Beers T. C., 2016, ApJ, 823, 30

Brook C. B., Kawata D., Gibson B. K., Freeman K. C., 2004, ApJ, 612, 894

Brook C. B. et al., 2012, MNRAS, 426, 690

Brown T. M. et al., 2010, ApJ, 725, L19 
Bureau M., Athanassoula E., 2005, ApJ, 626, 159

Calamida A. et al., 2014, ApJ, 790, 164

Casagrande L., Schönrich R., Asplund M., Cassisi S., Ramírez I., Meléndez J., Bensby T., Feltzing S., 2011, A\&A, 530, A138

Catchpole R. M., Whitelock P. A., Feast M. W., Hughes S. M. G., Irwin M., Alard C., 2016, MNRAS, 455, 2216

Cirasuolo M. et al., 2014, in Ramsay S. K., McLean I. S., Takami H., eds, Proc. SPIE Conf. Ser., Vol. 9147, Ground-based and Airborne Instrumentation for Astronomy V. SPIE, Bellingham, p. 91470N

Clarkson W. et al., 2008, ApJ, 684, 1110

Clarkson W. I. et al., 2011, ApJ, 735, 37

Cohen R. J., Few R. W., 1976, MNRAS, 176, 495

Cole D. R., Debattista V. P., Erwin P., Earp S. W. F., Roškar R., 2014, MNRAS, 445,3352

Combes F., Sanders R. H., 1981, A\&A, 96, 164

Combes F., Debbasch F., Friedli D., Pfenniger D., 1990, A\&A, 233, 82

de Jong R. S. et al., 2014, in Ramsay S. K., McLean I. S., Takami H., eds, Proc. SPIE Conf. Ser., Vol. 9147, Ground-based and Airborne Instrumentation for Astronomy V. SPIE, Bellingham, p. 91470M

de Vaucouleurs G., 1964, in Kerr F. J., ed., IAU Symp., Vol. 20, The Galaxy and the Magellanic Clouds. Kluwer, Dordrecht, p. 195

Debattista V. P., Sellwood J. A., 1998, ApJ, 493, L5

Debattista V. P., Sellwood J. A., 2000, ApJ, 543, 704

Debattista V. P., Carollo C. M., Mayer L., Moore B., 2005, ApJ, 628, 678

Debattista V. P., Mayer L., Carollo C. M., Moore B., Wadsley J., Quinn T., 2006, ApJ, 645, 209

Debattista V. P., Ness M., Earp S. W. F., Cole D. R., 2015, ApJ, 812, L16

Dékány I., Minniti D., Catelan M., Zoccali M., Saito R. K., Hempel M., Gonzalez O. A., 2013, ApJ, 776, L19

Di Matteo P. et al., 2014, A\&A, 567, A122

Di Matteo P. et al., 2015, A\&A, 577, A1

Dwek E. et al., 1995, ApJ, 445, 716

Erwin P., Beltrán J. C. V., Graham A. W., Beckman J. E., 2003, ApJ, 597, 929

Erwin P. et al., 2015, MNRAS, 446, 4039

Evans N. W., 1994, ApJ, 437, L31

Evans N. W., Belokurov V., 2002, ApJ, 567, L119

Ferreras I., Wyse R. F. G., Silk J., 2003, MNRAS, 345, 1381

Fisher D. B., Drory N., 2016, in Laurikainen E., Peletier R., Gadotti D., eds, Astrophysics and Space Science Library, Vol. 418, Galactic Bulges. Springer-Verlag, Switzerland, p. 41

Fux R., 1997, A\&A, 327, 983

Fux R., 1999, A\&A, 345, 787

Gadotti D. A., Seidel M. K., Sánchez-Blázquez P., Falcón-Barroso J., Husemann B., Coelho P., Pérez I., 2015, A\&A, 584, A90

Gardner E., Debattista V. P., Robin A. C., Vásquez S., Zoccali M., 2014, MNRAS, 438, 3275

Gerhard O., Martinez-Valpuesta I., 2012, ApJ, 744, L8

Gerhard O. E., Vietri M., 1986, MNRAS, 223, 377

Glazebrook K., 2013, PASA, 30, e056

Gnerucci A. et al., 2011, A\&A, 528, A88

Gonzalez O. A., Gadotti D., 2016, in Laurikainen E., Peletier R., Gadotti D., eds, Astrophysics and Space Science Library, Vol. 418, Galactic Bulges. Springer-Verlag, Switzerland, p. 199

Gonzalez O. A. et al., 2011, A\&A, 530, A54

Gonzalez O. A., Rejkuba M., Zoccali M., Valenti E., Minniti D., Schultheis M., Tobar R., Chen B., 2012, A\&A, 543, A13

Gonzalez O. A., Zoccali M., Debattista V. P., Alonso-García J., Valenti E., Minniti D., 2015, A\&A, 583, L5

Grand R. J. J., Springel V., Gómez F. A., Marinacci F., Pakmor R., Campbell D. J. R., Jenkins A., 2016, MNRAS, 459, 199

Grieco V., Matteucci F., Pipino A., Cescutti G., 2012, A\&A, 548, A60

Guedes J., Mayer L., Carollo M., Madau P., 2013, ApJ, 772, 36

Hammersley P. L., Garzón F., Mahoney T. J., López-Corredoira M., Torres M. A. P., 2000, MNRAS, 317, L45

Hill V. et al., 2011, A\&A, 534, A80
Holmberg J., Nordström B., Andersen J., 2009, A\&A, 501, 941

House E. L. et al., 2011, MNRAS, 415, 2652

Howard C. D., Rich R. M., Reitzel D. B., Koch A., De Propris R., Zhao H., 2008, ApJ, 688, 1060

Ivezic Z. et al., 2008, preprint (arXiv:0805.2366)

Johnson C. I., Rich R. M., Fulbright J. P., Valenti E., McWilliam A., 2011, ApJ, 732, 108

Johnson C. I., Rich R. M., Kobayashi C., Kunder A., Pilachowski C. A., Koch A., de Propris R., 2013, ApJ, 765, 157

Jurić M. et al., 2008, ApJ, 673, 864

Kassin S. A. et al., 2012, ApJ, 758, 106

Kauffmann G., White S. D. M., Guiderdoni B., 1993, MNRAS, 264, 201

Kennicutt R. C. Jr., Tamblyn P., Congdon C. E., 1994, ApJ, 435, 22

Kormendy J., Kennicutt R. C., 2004, ARA\&A, 42, 603

Kuijken K., Dubinski J., 1995, MNRAS, 277, 1341

Kuijken K., Rich R. M., 2002, AJ, 124, 2054

Kunder A. et al., 2012, AJ, 143, 57

Kunder A. et al., 2016, ApJ, 821, L25

Law D. R., Steidel C. C., Erb D. K., Larkin J. E., Pettini M., Shapley A. E., Wright S. A., 2009, ApJ, 697, 2057

Li Z., Gerhard O., Shen J., Portail M., Wegg C., 2016, ApJ, 824, 13

Li Z.-Y., Shen J., 2012, ApJ, 757, L7

Li Z.-Y., Shen J., 2015, ApJ, 815, L20

Liszt H. S., Burton W. B., 1980, ApJ, 236, 779

Loebman S. R., Roškar R., Debattista V. P., Ivezić, Ž., Quinn T. R., Wadsley J., 2011, ApJ, 737, 8

Loebman S. R., Debattista V. P., Nidever D. L., Hayden M. R., Holtzman J. A., Clarke A. J., Roškar R., Valluri M., 2016, ApJ, 818, L6

Majewski S. R. et al., 2016, Astron. Nachr., 337, 863

Martig M. et al., 2015, MNRAS, 451, 2230

Martinez-Valpuesta I., Gerhard O., 2011, ApJ, 734, L20

Martinez-Valpuesta I., Gerhard O., 2013, ApJ, 766, L3

Martinez-Valpuesta I., Shlosman I., Heller C., 2006, ApJ, 637, 214

McWilliam A., Zoccali M., 2010, ApJ, 724, 1491

Méndez-Abreu J., Debattista V. P., Corsini E. M., Aguerri J. A. L., 2014 A\&A, 572, A25

Merritt D., Sellwood J. A., 1994, ApJ, 425, 551

Minniti D. et al., 2010, New Astron., 15, 433

Mulder W. A., Liem B. T., 1986, A\&A, 157, 148

Nakada Y., Onaka T., Yamamura I., Deguchi S., Hashimoto O., Izumiura H., Sekiguchi K., 1991, Nature, 353, 140

Nataf D. M., Udalski A., Gould A., Fouqué P., Stanek K. Z., 2010, ApJ, 721, L28

Navarro J. F., Frenk C. S., White S. D. M., 1996, ApJ, 462, 563

Ness M. et al., 2012, ApJ, 756, 22

Ness M. et al., 2013a, MNRAS, 430, 836

Ness M. et al., 2013b, MNRAS, 432, 2092

Ness M., Debattista V. P., Bensby T., Feltzing S., Roškar R., Cole D. R., Johnson J. A., Freeman K., 2014, ApJ, 787, L19

Ness M., Hogg D. W., Rix H.-W., Martig M., Pinsonneault M. H., Ho A. Y. Q., 2016a, ApJ, 823, 114

Ness M. et al., 2016b, ApJ, 819, 2

Nidever D. L. et al., 2012, ApJ, 755, L25

Nikolaev S., Weinberg M. D., 1997, ApJ, 487, 885

Nordström B. et al., 2004, A\&A, 418, 989

O’Neill J. K., Dubinski J., 2003, MNRAS, 346, 251

Ortolani S., Renzini A., Gilmozzi R., Marconi G., Barbuy B., Bica E., Rich R. M., 1995, Nature, 377, 701

Paczynski B., Stanek K. Z., Udalski A., Szymanski M., Kaluzny J., Kubiak M., Mateo M., Krzeminski W., 1994, ApJ, 435, L113

Patel S. G. et al., 2013, ApJ, 778, 115

Peters W. L., III, 1975, ApJ, 195, 617

Quillen A. C., 2002, AJ, 124, 722

Quillen A. C., Minchev I., Sharma S., Qin Y.-J., Di Matteo P., 2014, MNRAS, 437,1284

Raha N., Sellwood J. A., James R. A., Kahn F. D., 1991, Nature, 352, 411

Raiteri C. M., Villata M., Navarro J. F., 1996, A\&A, 315, 105

Rojas-Arriagada A. et al., 2014, A\&A, 569, A103 
Saha K., Gerhard O., 2013, MNRAS, 430, 2039

Saha K., Martinez-Valpuesta I., Gerhard O., 2012, MNRAS, 421, 333

Sahu K. C. et al., 2006, Nature, 443, 534

Saito R. K., Zoccali M., McWilliam A., Minniti D., Gonzalez O. A., Hill V., 2011, AJ, 142, 76

Samland M., 2002, Ap\&SS, 281, 305

Schönrich R., Binney J., 2009, MNRAS, 399, 1145

Sellwood J. A., 1983, J. Comput. Phys., 50, 337

Sellwood J. A., 1985, MNRAS, 217, 127

Sevenster M. N., 1996, in Buta R., Crocker D. A., Elmegreen B. G., eds, ASP Conf. Ser. Vol. 91, IAU Colloq. 157: Barred Galaxies. Astron. Soc. Pac., San Francisco, p. 536

Shen J., Rich R. M., Kormendy J., Howard C. D., De Propris R., Kunder A., 2010, ApJ, 720, L72

Stadel J. G., 2001, PhD thesis, Univ. Washington

Stanek K. Z., Mateo M., Udalski A., Szymanski M., Kaluzny J., Kubiak M., 1994, ApJ, 429, L73

Stanek K. Z., Udalski A., Szymanski M., Kaluzny J., Kubiak M., Mateo M., Krzeminski W., 1997, ApJ, 477, 163

Stinson G. S. et al., 2013, MNRAS, 436, 625

Tacchella S. et al., 2015, Science, 348, 314

Terrazas B. A., Bell E. F., Henriques B. M. B., White S. D. M., 2016, MNRAS, 459, 1929

Thielemann F.-K., Nomoto K., Yokoi K., 1986, A\&A, 158, 17

Uttenthaler S., Schultheis M., Nataf D. M., Robin A. C., Lebzelter T., Chen B., 2012, A\&A, 546, A57

Valenti E., Zoccali M., Renzini A., Brown T. M., Gonzalez O. A., Minniti D., Debattista V. P., Mayer L., 2013, A\&A, 559, A98

van Dokkum P. G. et al., 2013, ApJ, 771, L35

Wadsley J. W., Stadel J., Quinn T., 2004, New Astron., 9, 137

Walker A. R., 1989, PASP, 101, 570

Wegg C., Gerhard O., 2013, MNRAS, 435, 1874

Wegg C., Gerhard O., Portail M., 2015, MNRAS, 450, 4050

Weidemann V., 1987, A\&A, 188, 74

Weiland J. L. et al., 1994, ApJ, 425, L81

Weinberg M. D., 1985, MNRAS, 213, 451

Whitelock P., Catchpole R., 1992, in Blitz L., ed., Astrophysics and Space Science Library, Vol. 180, The Center, Bulge, and Disk of the Milky Way. Springer-Verlag, Dordrecht, Netherlands, p. 103

Widrow L. M., Dubinski J., 2005, ApJ, 631, 838

Widrow L. M., Pym B., Dubinski J., 2008, ApJ, 679, 1239

Wielen R., 1977, A\&A, 60, 263

Wisnioski E. et al., 2015, ApJ, 799, 209

Wyatt S. P., Cahn J. H., 1983, ApJ, 275, 225

Zhao H., Rich R. M., Spergel D. N., 1996, MNRAS, 282, 175

Zoccali M. et al., 2003, A\&A, 399, 931

Zoccali M., Hill V., Lecureur A., Barbuy B., Renzini A., Minniti D., Gómez A., Ortolani S., 2008, A\&A, 486, 177

Zoccali M. et al., 2014, A\&A, 562, A66

Zoccali M. et al., 2017, A\&A, 599, A12

\section{APPENDIX A: HEIGHT DEPENDENCE OF ORBITAL FREQUENCIES}

Here, we demonstrate that the vertical frequency, $v$, of a star declines more rapidly with increasing maximum height, $z_{\max }$, than does the orbital frequency, $\Omega$. We do this directly by computing orbits of stars in the pure $N$-body model with disc D5 described in Section 3. We select all stars in a narrow annulus of guiding radius $3.95 \leq$ $R_{\text {gui }} /$ kpc $\leq 4.05$, where $R_{\text {gui }}=J_{z} / V_{c}, J_{z}$ is the angular momentum of the particle about the $z$-axis, and $V_{c}$ is the circular velocity at

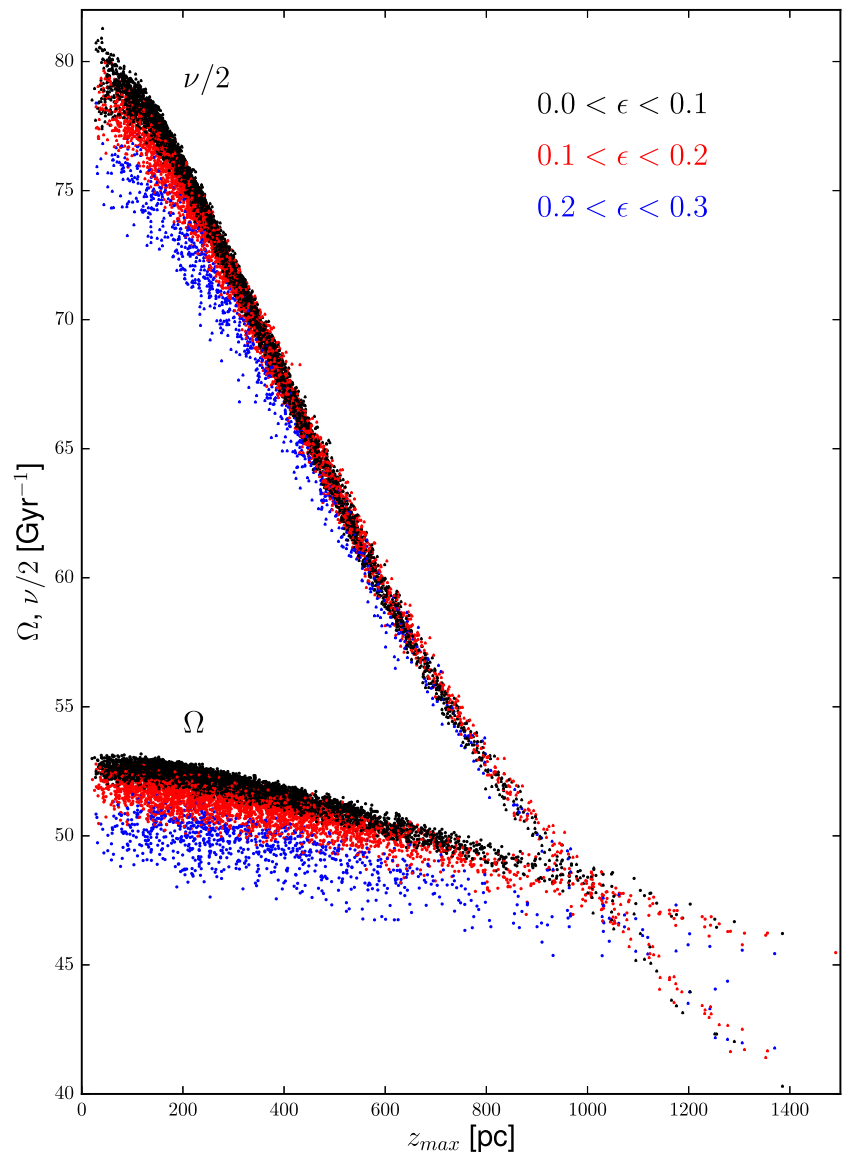

Figure A1. Filled triangles show $v / 2$, while filled circles show $\Omega$ for individual star particles. Black, red and blue points show increasing eccentricity as indicated. All orbits have guiding radius $3.95 \leq R_{\text {gui }} / \mathrm{kpc} \leq 4.05$.

this radius. We choose this annulus because the peanut shape is very distinct at the end of the simulation in this region; however the vertical distribution of the stars is much narrower than the final peanut shape. We integrate orbits for $\sim 9000$ of these particles, using a time-step of $10^{4} \mathrm{yr}$, saving the phase space coordinates of each star every time-step over a $1 \mathrm{Gyr}$ period. From these orbits, we then compute the eccentricity of the orbit

$\epsilon=\frac{R_{\max }-R_{\min }}{R_{\max }+R_{\min }}$

where $R_{\max }$ and $R_{\min }$ are the maximum and minimum radius reached; $\epsilon=0$ corresponds to a circular orbit. We also compute $z_{\max }$ as well as the tangential and vertical frequencies, $\Omega$ and $\nu$, respectively.

Fig. A1shows the result of this calculation. It is very clear that $v / 2$ declines much more rapidly with $z_{\max }$ than does $\Omega$, which only changes by $\sim 3 \mathrm{Gyr}^{-1}$ over this vertical range. The figure also shows the variation of $\Omega$ and $\nu / 2$ as a function of $\epsilon$. The angular frequency $\Omega$ decreases with increasing $\epsilon$, while the change in $\nu / 2$ with $\epsilon$ is more modest.

This paper has been typeset from a $\mathrm{T}_{\mathrm{E}} \mathrm{X} / \mathrm{LT} \mathrm{E} \mathrm{X}$ file prepared by the author. 